Lorena Bociu · Giovanna Guidoboni ·

Riccardo Sacco · Justin T. Webster

\title{
Analysis of nonlinear poro-elastic and poro-visco-elastic models
}

The first author was partially supported by the National Science Foundation with grant NSFDMS 1312801. The second author was partially supported by the National Science Foundation with grants NSF-DMS 1134731 and 1224195 and by the chair Gutenberg funds of the Cercle Gutenberg of the Region Alsace (France). The fourth author was partially supported by the National Science Foundation with grant NSF-DMS-1504697.

\author{
L. Bociu \\ Department of Mathematics \\ North Carolina State University \\ SAS Hall 3236 \\ 27695 Raleigh, NC, USA \\ Tel.: +1 919-515-2370 \\ Fax: + 1919-513-7336 \\ E-mail: lvbociu@ncsu.edu \\ G. Guidoboni \\ Department of Mathematical Sciences \\ Indiana University Purdue University Indianapolis \\ 402 N. Blackford St, 46202 Indianapolis, IN, USA \\ Tel.: +1 317-274-6936 \\ Fax: + $1317-274-3460$ \\ E-mail: gguidobo@math.iupui.edu \\ R. Sacco \\ Dipartimento di Matematica \\ Politecnico di Milano \\ Via Bonardi 9, 20133 Milano, Italy \\ Tel.: +3902 2399-4540 \\ Fax: +39 02 2399-4513 \\ E-mail: riccardo.sacco@polimi.it \\ J.T. Webster \\ Department of Mathematics \\ College of Charleston \\ 66 George St, 29424 Charleston, SC, USA \\ Tel.: +1 $843953-1040$ \\ Fax: $+1843953-1410$ \\ E-mail: websterj@cofc.edu
}

This is the author's manuscript of the article published in final edited form as:

Bociu, L., Guidoboni, G., Sacco, R., \& Webster, J. T. (2016). Analysis of nonlinear poro-elastic and poro-viscoelastic models. Archive for Rational Mechanics and Analysis, 222(3), 1445-1519. 


\begin{abstract}
We consider the initial and boundary value problem for a system of partial differential equations describing the motion of a fluid-solid mixture under the assumption of full saturation. The ability of the fluid phase to flow within the solid skeleton is described by the permeability tensor, which is assumed here to be a multiple of the identity and to depend nonlinearly on the volumetric solid strain. In particular, we study the problem of existence of weak solutions in bounded domains, accounting for non-zero volumetric and boundary forcing terms. We investigate the influence of viscoelasticity on the solution functional setting and on the regularity requirements for the forcing terms. The theoretical analysis shows that different time regularity requirements are needed for the volumetric source of linear momentum and the boundary source of traction depending on whether or not viscoelasticity is present. The theoretical results are further investigated via numerical simulations based on a novel dual mixed hybridized finite element discretization. When the data are sufficiently regular, the simulations show that the solutions satisfy the energy estimates predicted by the theoretical analysis. Interestingly, the simulations also show that, in the purely elastic case, the Darcy velocity and the related fluid energy might become unbounded if indeed the data do not enjoy the time regularity required by the theory.
\end{abstract}

\title{
1 Introduction
}

In this paper we consider a nonlinear system of partial differential equations (PDEs) often encountered when modeling fluid flow through deformable porous media. Historically, petroleum engineering has been the main applied field driving the theoretical development of porous media flow [3,17]. More recently, similar approaches have been applied to the modeling of fluid flow through biological tissues, with applications spanning from bio-engineering $[16,32,38]$ to physiology $[9,11,28]$.

Mechanical properties of biological tissues differ significantly from those of rocks. In particular, since most of biological tissues are composed by both elastin and collagen, the deformable matrix within the porous medium exhibits both elastic and visco-elastic behaviors [22]. Interestingly, material properties and volume fractions of elastic and collagen vary in health and disease, thereby motivating a detailed investigation of their influence on the physical system and, consequently, on the solution of the PDEs describing this system

The precise system considered in this paper is described in Section 2. The mathematical model describes the motion of a fluid-solid mixture under the assumption of full saturation. The ability of the fluid phase to flow within the solid skeleton is described by the permeability tensor, which is assumed here to be a multiple of the identity and to depend nonlinearly on the volumetric solid strain. We study the problem of existence of weak solutions in bounded domains, accounting for non-zero volumetric and boundary forcing terms. Specifically, we consider mixed boundary conditions, including the case where the Dirichlet and Neumann portions of the boundary may intersect. We investigate the influence of viscoelasticity on the solution functional setting and on the regularity requirements for the forcing terms. The results obtained via the theoretical analysis are further explored via numerical simulations of one-dimensional test cases that serve as 
simplified benchmark examples while retaining the main features of the full problem, in particular the nonlinearity in the permeability constitutive equation.

Several theoretical approaches have been developed to study poroelastic systems, as briefly reviewed in Section 3.1. However, to the best of our knowledge, this article presents the first study that simultaneously accounts for non-zero, mixed boundary data, nonlinear dependence of the permeability on the volumetric solid strain, and elastic and viscoelastic effects in the solid component. Although it is true that viscoelasticity provides some additional time regularity of the displacement, it does not necessarily simplify the analysis. Rather, in some instances it brings up new technical points that must be addressed, as discussed in Section 3.1. The computational method used to investigate numerically the theoretical findings is also a novel contribution of this article. The algorithm combines a Backward Euler method for the discretization in time, a dual mixed hybridized finite element method for the discretization in space and a fixed-point iteration for the nonlinearity in the permeability which couples fluid and solid equations. The proposed numerical method avoids direct differentiation in the computation of gradient quantities which appear in the definition of the energies provided by the theoretical analysis, thereby allowing for high accuracy in the simulation results.

The main existence results are provided by Theorems 1 and 2 for the viscoelastic and purely elastic case, respectively. It is interesting to notice the different requirements for the time regularity of the volumetric source of linear momentum and the boundary source of traction, namely $L^{2}$ time regularity for the viscoelastic case and $H^{1}$ for the elastic case. Interestingly, our numerical investigation shows that the Darcy velocity and the related fluid energy might become unbounded in the purely elastic case if the data do not enjoy sufficient time regularity.

The paper is organized as follows. In Section 2 we describe the mathematical model considered in the article and its interpretation from the engineering viewpoint. The theoretical study on the existence of solutions is presented in Section 3, whereas the numerical method and the simulation results are discussed in Section 4. Conclusions are outlined in Section 5.

\section{Mathematical model}

\subsection{Balance equations}

Let $\Omega$ be an open subset of $\mathbb{R}^{3}$ representing the spatial domain occupied by the fluid-solid mixture, and let $\mathbf{x}$ be the position vector of each point in the body with respect to a fixed Cartesian reference frame. The symbol $\mathbf{n}$ will be used to denote the unit normal vector to $\Omega$. Let $V_{s}(\mathbf{x}, t)$ and $V_{f}(\mathbf{x}, t)$ be the volumes occupied by the solid and the fluid components, respectively, in every representative elementary volume $V(\mathbf{x}, t)$ centered at $\mathbf{x} \in \Omega$ at time $t$. Then, the volumetric fraction $\phi$ of the fluid component, also called porosity, and its increment $\zeta$ with respect to its baseline value $\phi_{0}$, also called fluid content, are defined as

$$
\phi(\mathbf{x}, t)=\frac{V_{f}(\mathbf{x}, t)}{V(\mathbf{x}, t)} \quad \text { and } \quad \zeta(\mathbf{x}, t)=\phi(\mathbf{x}, t)-\phi_{0}(\mathbf{x}) .
$$

Under the assumption of fully saturated mixture, the volumetric fraction of the solid component is given by $1-\phi(\mathbf{x}, t)$. Moreover, under the assumptions of neg- 
ligible inertia, small deformations and intrinsic incompressibility of each mixture component $[1,21,30,34,43]$ the motion of the poro-elastic material is governed by the following equations for the balance of mass (of the fluid component) and linear momentum (for the fluid-solid mixture):

$$
\zeta_{t}+\nabla \cdot \mathbf{v}=S(\mathbf{x}, t) \quad \text { and } \quad \nabla \cdot \mathbf{T}+\mathbf{F}=\mathbf{0} \quad \text { in } \Omega \times(0, T)
$$

where $\mathbf{T}$ is the stress tensor of the mixture (also known as total stress), $\mathbf{v}$ is the discharge velocity, $\mathbf{F}$ is a body force per unit of volume and $S$ is a net volumetric fluid production rate. Here the partial derivative with respect to time has been denoted by the subscript $t$. This notation will be used throughout the paper.

Remark 1 In continuum mechanics, the source terms $S$ and $\mathbf{F}$ should be written as

$$
S=\phi S_{f}, \quad \mathbf{F}=\rho \mathbf{f}-\rho_{f} S_{f} \mathbf{v}-\rho_{f} \phi S_{f} \mathbf{u}_{t},
$$

where $\rho=\rho_{f} \phi+\rho_{s}(1-\phi)$ is the density of the mixture, $\rho_{f}$ and $\rho_{s}$ are the specific densities of the fluid and solid components, $\mathbf{u}$ is the solid displacement field and $S_{f}$ and $\mathbf{f}$ are given data. Our analysis is performed under the simplifying assumption that $S$ and $\mathbf{F}$ (rather than $S_{f}$ and $\mathbf{f}$ ) are given functions. This assumption is justified by the facts that $(i)$ the few existing theoretical studies accounting for non-zero mass and momentum sources in poro-elastic systems adopt the same assumption, see [6,51,55] and references therein, and (ii) assuming $S$ and $\mathbf{F}$ given is a necessary preliminary step towards the more realistic case where $S_{f}$ and $\mathbf{f}$ are prescribed.

\subsection{Constitutive equations}

The balance equations are completed with the following constitutive equations for the total stress, the discharge velocity and the fluid content:

1. total stress:

$$
\mathbf{T}=\mathbf{T}_{e}+\delta \mathbf{T}_{v}-p \mathbf{I},
$$

where $\mathbf{T}_{e}$ and $\mathbf{T}_{v}$ are the elastic and viscoelastic stress contributions, respectively, defined as

$$
\mathbf{T}_{e}=2 \mu_{e} \varepsilon(\mathbf{u})+\lambda_{e}(\nabla \cdot \mathbf{u}) \mathbf{I} \quad \text { and } \quad \mathbf{T}_{v}=2 \mu_{v} \varepsilon\left(\mathbf{u}_{t}\right)+\lambda_{v}\left(\nabla \cdot \mathbf{u}_{t}\right) \mathbf{I},
$$

where $\varepsilon(\mathbf{w})$ is the symmetric part of the gradient of the vector field $\mathbf{w}$, namely $\varepsilon(\mathbf{w})=\left(\nabla \mathbf{w}+\nabla \mathbf{w}^{T}\right) / 2, p$ is the Darcy fluid pressure, $\mathbf{u}$ is the solid displacement, $\mathbf{I}$ is the identity tensor, $\lambda_{e}$ and $\mu_{e}$ are the Lamé elastic parameters, and $\lambda_{v}$ and $\mu_{v}$ are the viscoelastic parameters. The parameter $\delta \geq 0$ indicates the extent to which the model includes viscoelastic effects for the solid component, with $\delta=0$ corresponding to the purely elastic case; 
2. discharge velocity:

$$
\mathbf{v}=-\mathbf{K} \nabla p, \quad \text { with } \quad \mathbf{K}=k \mathbf{I} \quad \text { and } \quad k=k_{r e f} f_{k}(\phi)
$$

where $\mathbf{K}$ is the permeability tensor, and $k_{r e f}$ is a reference value for the permeability of the mixture. Here we assume that $\mathbf{K}$ depends on the porosity and that is a multiple of the identity tensor. The particular form of the relationship between the permeability $k$ and the porosity $\phi$ is represented by the function $f_{k}(\phi)$ and it depends on the geometrical architecture of the pores inside the matrix and the physical properties of the fluid. Many studies have considered $k$ to be constant, i.e. $f_{k}(\phi)=1$ and $k=k_{r e f}$, leading to a linear coupling between the equations for linear momentum and mass balance. However, in many applications $k$ is definitely not a constant. For example, if a Newtonian fluid flows in the interstitial spaces of a pack of spherical particles, then the Carman-Kozeny formula states that

$$
k_{r e f}=\frac{C_{c k}}{\mu_{f}}, \quad f_{k}(\phi)=\frac{\phi^{3}}{(1-\phi)^{2}},
$$

where $C_{c k}$ is a constant depending on the geometric properties of the pack of particles and $\mu_{f}$ is the fluid viscosity [26]. On the other hand, if a Newtonian fluid flows inside cylindrical pores, then the formula for capillary beds states that

$$
k_{\text {ref }}=\frac{C_{c b}}{\mu_{f}}, \quad f_{k}(\phi)=\phi^{2},
$$

where $C_{c b}$ is a constant depending on the geometric properties of the capillary bed and $\mu_{f}$ is the fluid viscosity [9]. The theoretical analysis in Section 3 is performed without specifying a particular expression for $k$, but assuming that $k$ is bounded (See Assumption 3.1). In the numerical study presented in Section 4, simulations are performed using the Carman-Kozeny permeability law, where upper and lower bounds have been artificially imposed to meet the theoretical Assumption 3.1;

\section{3. fluid content:}

$$
\zeta=\nabla \cdot \mathbf{u}, \quad \text { implying that } \quad \phi=\phi_{0}+\nabla \cdot \mathbf{u}
$$

We remark that equation (9) is a particular instance of the more general expression $\zeta=c_{0} p+\alpha \nabla \cdot \mathbf{u}$, where $c_{0}$ is the constrained specific storage coefficient and $\alpha$ is the Biot-Willis coefficient. Under the assumption of incompressibility for the fluid and solid components of the mixture (cf. [17]), the coefficients reduce to $c_{0}=0$ and $\alpha=1$. As a consequence, the permeability $k$ reduces to be a function of $\nabla \cdot \mathbf{u}$ only (rather than a function of both $p$ and $\nabla \cdot \mathbf{u}$ ). Thus, $k=k(\phi)=k(\phi(\nabla \cdot \mathbf{u}))$ is abbreviated in the following theoretical and numerical analysis as $k=k(\nabla \cdot \mathbf{u})$. 


\subsection{Boundary conditions}

Let us denote by $\partial \Omega=\overline{\Gamma_{D}} \cup \overline{\Gamma_{N}}$ the boundary of $\Omega$, with $\Gamma_{D}=\Gamma_{D, p} \cup \Gamma_{D, v}$ and $\bar{\Gamma}_{D} \cap \bar{\Gamma}_{N}$ possibly nonempty. We consider the following boundary conditions:

$$
\begin{array}{ccc}
\mathbf{T n}=\mathbf{g}, & \mathbf{v} \cdot \mathbf{n}=0 & \text { on } \Gamma_{N} \\
\mathbf{u}=\mathbf{0}, & p=0 & \text { on } \Gamma_{D, p}, \\
\mathbf{u}=\mathbf{0}, & \mathbf{v} \cdot \mathbf{n}=\psi & \text { on } \Gamma_{D, v} .
\end{array}
$$

Here $\mathbf{g}$ and $\psi$ are given functions of space and time. The subscripts in the boundary partition reflect the type of associated boundary conditions. More precisely, the subscripts $N$ and $D$ indicate conditions imposed on stress and displacement, respectively, whereas the subscripts $p$ and $v$ indicate conditions imposed on Darcy pressure and velocity, respectively.

\subsection{Initial conditions}

In order to specify the initial conditions, it is useful to distinguish between the viscoelastic case, i.e. $\delta>0$, and the purely elastic case, i.e. $\delta=0$.

When $\delta>0$, time derivatives appear in both the equations for linear momentum and mass balance, requiring an initial condition on the whole displacement field, namely

$$
\mathbf{u}=\mathbf{u}^{0} \quad \text { in } \Omega \quad \text { at } t=0 \quad(\text { case } \delta>0) .
$$

When $\delta=0$, only the fluid content $\zeta$ in the mass balance equation undergoes time differentiation. Since $\zeta=\nabla \cdot \mathbf{u}$ by equation (9), only a condition on $\nabla \cdot \mathbf{u}$ is required, namely

$$
\nabla \cdot \mathbf{u}=d_{0} \quad \text { in } \Omega \quad \text { at } t=0 \quad(\text { case } \delta=0) .
$$

However, in order to obtain a priori estimates for the solutions in the case $\delta=$ 0 , we will need to consider only those $d_{0}$ for which there exist an $\mathbf{u}^{0}$ such that $\nabla \cdot \mathbf{u}^{0}=d_{0}$, as explained in Remark 3 .

\section{Existence of solutions}

\subsection{Main challenges and related literature}

The mathematical model described in Section 2 has inspired many theoretical investigations. The two-dimensional linear problem with constant permeability and without viscoelastic effects is addressed in [55]. This fundamental work studies a weak form of the problem (and associated solutions); a version of Rothe's method is utilized, which involves both temporal and spatial discretization. The work in [55] provides a general strategy for the linear analysis (especially in the $\delta=0$ case), and we follow the conventions presented therein. However, in our case, the well-posedness analysis is greatly complicated by the presence of nonlinear 
coupling via the permeability $k=k(\nabla \cdot \mathbf{u})$, here depending on the dilation of the structure. Additionally, uniqueness comes easily in the linear dynamics and does not depend on the regularity properties of the solution nor the dimension of the space; this is certainly not the case for the dynamics considered here.

A linear elastic version of the model in Section 2 is also considered in [40], but with a different (and stronger) notion of solution than that in [55]. In [40] a Galerkin method is proposed for purely homogeneous boundary conditions for the pressure and displacement. This allows for a nice notion of strong solution coming from the viability of smoother test functions. For this linear result, the (null) Dirichlet boundary conditions are critical since the proof of the main theorem therein requires elliptic regularity [40, p.44] (an issue with which we must contend below).

Another fundamental work on the linear Biot dynamics is [51]. In this study, the author develops a functional framework for the dynamics of the system, in the context of semigroup theory for implicit evolution equations. This approach accommodates general boundary conditions, as well as effects due to partial saturation. Various well-posedness theorems are developed (depending on parameter values) and notions of "strong" and "weak" solutions are discussed in relation to the various notions of differentiability for the fundamental quantities. This paper also deals with an effect known as secondary consolidation, which is pertinent in geoscience applications. Although this involves an additional (time-differentiated) term in the elasticity equation, the work therein does not fully address the effects of viscoelasticity on the solutions as we do here. Two subsequent papers address nonlinear effects in the Biot model above. Specifically, [54] addresses a (monotone, nonlinear) permeability depending on pressure (rather than dilation, as in our model); the analysis there seems motivated by geoscience applications, and the techniques do not generalize to the nonlinear coupling considered in the present paper (where the monotonicity property is lost). A second nonlinear paper [52] incorporates nonlinear plasticity into the model (which may allow for hysteresis effects). In some sense, the results there allow for a semigroup generation for a linear model incorporating viscoelastic effects.

The study presented in [6] is the first contribution (to our knowledge) addressing the nonlinear Biot model (without viscoelasticity) illustrated in Section 2, with permeability depending on dilation. However, the analysis is performed in the case of null boundary conditions for both pressure and elastic displacement. The boundary consists of a single piece upon which zero Dirichlet conditions are prescribed (the approach, as in [51], allows for inhomogeneous terms via a translation argument). The strategy in [6] relies on the Rothe's method (as in [55]), but uses the simplified structure of the pressure-to-dilation operator $B$ (see Section 3.3 below) coming from [51]. Existence of solutions is shown (in a weak sense, similar, though not identical, to [55]); uniqueness is addressed via strong assumptions on the a priori regularity of the pressure, and the structure of the permeability. Some numerical results are also provided. The analysis in [6] is illustrative in its handling of the nonlinear coupling, but is simplified in comparison to the analysis here by considering homogeneous clamped/Dirichlet boundary conditions. In our analysis we consider physical boundary conditions coming from applications (non-homogeneous, mixed Dirichlet-Neumann boundary conditions) and address the associated technical issues. Moreover, we provide a unified framework for 
both the elastic and viscoelastic cases, along with the associated energy estimates and identities (when available). Our assumptions on the permeability (with respect to the existence of solutions) mirror those in [6], and are motivated by many biological and biomedical applications, see e.g. [9,11,16,28,32,38].

Subsequent papers [7] and [8] address the stationary problem, where meaningful statements can be made about uniqueness of solutions and associated regularity (the two issues not being unrelated). These papers allow for more general boundary conditions, and consider other notions of solutions, but the techniques seem to be restricted to the stationary case (steady flows). The papers in [6-8] do not appear to have specific applications in mind, and thus provide general analysis and some corresponding numerical results.

Our work complements, extends, and (in some sense) goes beyond the aforementioned studies, in the sense that:

- We consider a physical problem with no simplifying assumptions on the domain boundary, (i.e., we include the case when $\bar{\Gamma}_{D} \cap \bar{\Gamma}_{N} \neq \emptyset$ ), and the associated boundary conditions (i.e., Neumann and Dirichlet for both solid and fluid components). This leads to an elliptic problem for the Lamé system with mixed boundary conditions. Other analyses of the nonlinear Biot model above do not seem to accommodate such conditions, and for many biological and biomedical applications boundary data are the fundamental drivers of system dynamics.

- We address the critical need of elliptic regularity (corresponding to the stationary elasticity problem with mixed boundary conditions) in the construction of solutions. In previous studies, boundary conditions were not the focus in the well-posedness analysis.

- We allow for fully general $\Omega$-distributed forces, as well as Dirichlet/Neumann data, for both the pressure and displacement dynamics. We track the regularity properties of the data and note their effect on the solutions. Additionally, we note that such effects vary between the elastic and viscoelastic cases, as discussed in Remark 5 and shown in the simulation results in Section 4.6.

- Our approach accounts for viscoelastic effects in the solid, i.e. $\delta>0$, but also allows to study the purely elastic case, i.e. $\delta=0$. Our investigation is motivated by the fact that the viscoelastic properties of biological tissues often vary with age and disease, and, interestingly, our analysis shows that the system dynamics fundamentally changes as viscoelastic effects vanish. 
3.2 Preliminary notions and definition of solutions

For the theoretical analysis, it is useful to rewrite the problem as follows:

$$
\begin{array}{cc}
\nabla \cdot \mathbf{T}(\mathbf{u}, p)=-\mathbf{F} & \text { in } \Omega \times(0, T) \\
\nabla \cdot \mathbf{u}_{t}-\nabla \cdot(k(\nabla \cdot \mathbf{u}) \nabla p)=S & \text { in } \Omega \times(0, T) \\
\nabla \cdot \mathbf{u}=d_{0} & \text { in } \Omega, \text { for } t=0 \\
\mathbf{T}(\mathbf{u}, p) \mathbf{n}=\mathbf{g} & \text { on } \Gamma_{N} \times(0, T) \\
\mathbf{u}=\mathbf{0} & \text { on } \Gamma_{D} \times(0, T) \\
\nabla p \cdot \mathbf{n}=0 & \text { on } \Gamma_{N} \times(0, T) \\
p=0 & \text { on } \Gamma_{D, p} \times(0, T) \\
-k(\nabla \cdot \mathbf{u}) \nabla p \cdot \mathbf{n}=\psi & \text { on } \Gamma_{D, v} \times(0, T)
\end{array}
$$

where

$$
\mathbf{T}(\mathbf{u}, p)=[2 \varepsilon(\mathbf{u})+(\nabla \cdot \mathbf{u}) \mathbf{I}]+\delta\left[2 \varepsilon\left(\mathbf{u}_{t}\right)+\left(\nabla \cdot \mathbf{u}_{t}\right) \mathbf{I}\right]-p \mathbf{I}
$$

and where the Lamé elastic parameters and the viscoelastic coefficients have been normalized to unity and where the source terms in the volume, i.e. $\mathbf{F}$ and $S$, and on the boundary, i.e. $\mathbf{g}$ and $\psi$, are given functions of space and time. We remark that the normalization of parameters to unity is not essential to the analysis, but it significantly simplifies the description of the steps in the existence proof. For the sake of completeness, the theoretical estimates are reported for the non-normalized physical parameters in Section 3.6.

Notation: Norms $\|\cdot\|$ are taken to be $L^{2}(D)$ for a domain $D$. Inner products in $L^{2}(D)$ are written as $(\cdot, \cdot)$, whereas $\langle\cdot, \cdot\rangle$ will denote the inner product on the boundary $L^{2}(\partial D)$. A subscript will denote the domain where the context does not immediately make it clear, e.g. $\langle\mathbf{u}, \mathbf{w}\rangle_{\Gamma_{N}}$. The Sobolev space of order $s$ defined on a domain $D$ will be denoted by $H^{S}(D)$, with $H_{0}^{S}(D)$ denoting the closure of $C_{0}^{\infty}(D)$ in the $H^{s}(D)$ norm (which we denote by $\|\cdot\|_{H^{s}(D)}$ or $\|\cdot\|_{s, D}$ ). When $s=0$ we may further abbreviate the notation to $\|\cdot\|$ (as described above). We make use of the standard notation for the trace of functions $\gamma[w]$ as the map from $H^{1}(D)$ to $H^{1 / 2}(\partial D)$. We will make use of the spaces $L^{2}(0, T ; U)$ and $H^{s}(0, T ; U)$, where $U$ is a topological vector space. These norms (and associated inner products) will be denoted with the appropriate subscript, e.g., $\|\cdot\|_{L^{2}(0, T ; U)}$.

The principal spaces we consider are of the form

$$
H_{\Gamma_{*}}^{1}(\Omega)=\left\{f \in H^{1}(\Omega):\left.\gamma[f]\right|_{\Gamma_{*}}=0\right\} .
$$

In this case we have $H_{\Gamma_{*}}^{1}(\Omega) \supset H_{0}^{1}(\Omega)$ for any $\Gamma_{*} \subset \Gamma \equiv \partial \Omega$. The primary spaces in our analysis below are

$$
V \equiv H_{\Gamma_{D, p}}^{1}, \quad \mathbf{V} \equiv\left(H_{\Gamma_{D}}^{1}(\Omega)\right)^{3}, \quad \mathbb{V} \equiv V \times \mathbf{V},
$$

for the pressure $p$ and displacement $\mathbf{u}$, respectively. The norms in these spaces are inherited from $H^{1}(\Omega)$ and $\left(H^{1}(\Omega)\right)^{3}$, respectively. . For simplicity we will introduce a bilinear form associated with the elasticity operator:

$$
a(\mathbf{u}, \mathbf{w})=(\nabla \cdot \mathbf{u}, \nabla \cdot \mathbf{w})+(\nabla \mathbf{u}, \nabla \mathbf{w})+\left(\nabla \mathbf{u},(\nabla \mathbf{w})^{T}\right) .
$$


In this notation, we interpret $\nabla \mathbf{u}$ as the Jacobian of $\mathbf{u}$, i.e. $(\nabla \mathbf{u})_{i j}=D_{j} u_{i}$, and we utilize the Frobenius scalar product:

$$
(\mathbf{A}, \mathbf{B})=\int_{\Omega}\left(A_{i j} B_{i j}\right) d \Omega,
$$

sometimes also denoted by $(\mathbf{A}: \mathbf{B})$. Notice that, when $\mathbf{A}=\mathbf{B}$, we write $(\mathbf{A}: \mathbf{A})=$ $\left(A_{i j}, A_{i j}\right)=\|\mathbf{A}\|^{2}$. We topologize the space $\mathbf{V}$ via $a(\cdot, \cdot)$, which is to say that we take the norm induced by $a(\cdot, \cdot)$ as the norm on $\mathbf{V}$ (see Assumption 3.1 below).

In the case of constant permeability, the model is linear, as in [40], and one has access to both a "strong" notion of solution (classically differentiable in time) and a "weak" notion of solutions (only distributionally differentiable in time). Here, our notion of solution depends critically on the parameter $\delta$. We dispense with the usage of the words "strong" and "weak" for solutions, owing to the confusion it causes with the associated weak forms of the solutions. In both cases $\delta>0$ (viscoelastic case, or VE) and $\delta=0$ (elastic case, or E), solutions will satisfy a weak form of (15)-(22). Our notion of an E-solution $(\delta=0)$ here follows that in [55] (and it is closely related to the notion in [6]). For a VE-solution $(\delta>0)$, we extend this notion in a natural way as specified below.

Definition 1 [VE-Solution] A solution to (15)-(22) (with $\delta>0$ ) is represented by the pair of functions $\mathbf{u} \in H^{1}(0, T ; \mathbf{V})$ and $p \in L^{2}(0, T ; V)$ such that:

(a) the following relations are satisfied for any $\mathbf{w} \in \mathbf{V}, q \in V$, and $f \in C^{\infty}((0, T))$ :

$$
\begin{aligned}
\delta \int_{0}^{T} a\left(\mathbf{u}_{t}, \mathbf{w}\right) f d t+\int_{0}^{T} a(\mathbf{u}, \mathbf{w}) f d t-\int_{0}^{T}(p, \nabla \cdot \mathbf{w}) f d t & \\
& =\int_{0}^{T}\langle\mathbf{g}, \mathbf{w}\rangle_{\Gamma_{N}} f d t-\int_{0}^{T}(\mathbf{F}, \mathbf{w}) f d t \\
\int_{0}^{T}(k(\nabla \cdot \mathbf{u}) \nabla p, \nabla q) f d t & +\int_{0}^{T}\left(\nabla \cdot \mathbf{u}_{t}, q\right) f d t \\
& =-\int_{0}^{T}\langle\psi, q\rangle_{\Gamma_{D, v}} f d t+\int_{0}^{T}(S, p) f d t
\end{aligned}
$$

(b) the initial conditions $\mathbf{u}(\mathbf{x}, 0)=\mathbf{u}^{0} \in \mathbf{V}$ and $\nabla \cdot \mathbf{u}(\mathbf{x}, 0)=d_{0} \in L^{2}(\Omega)$ are given, and we require $\nabla \cdot \mathbf{u}^{0}=d_{0}$ (in the $L^{2}(\Omega)$ sense).

Definition 2 [E-Solution] A solution to (15)-(22) (with $\delta=0$ ) is represented by the pair of functions $\mathbf{u} \in L^{2}(0, T ; \mathbf{V})$ and $p \in L^{2}(0, T ; V)$ such that:

(a) the following relations are satisfied for any $\mathbf{w} \in \mathbf{V}, q \in V$, and $f \in C_{0}^{\infty}((0, T))$ :

$$
\begin{gathered}
\int_{0}^{T} a(\mathbf{u}, \mathbf{w}) f d t-\int_{0}^{T}(p, \nabla \cdot \mathbf{w}) f d t \\
\quad=\int_{0}^{T}\langle\mathbf{g}, \mathbf{w}\rangle_{\Gamma_{N}} f d t-\int_{0}^{T}(\mathbf{F}, \mathbf{w}) f d t \\
\int_{0}^{T}(k(\nabla \cdot \mathbf{u}) \nabla p, \nabla q) f d t-\int_{0}^{T}(\nabla \cdot \mathbf{u}, q) f^{\prime} d t \\
=-\int_{0}^{T}\langle\psi, q\rangle_{\Gamma_{D, v}} f d t+\int_{0}^{T}(S, p) f d t
\end{gathered}
$$


(b) for every $q \in V$, the term $(\nabla \cdot \mathbf{u}(t), q)$ uniquely defines an absolutely continuous function on $[0, T]$ and the initial condition $(\nabla \cdot \mathbf{u}(0), q)=\left(d_{0}, q\right)$ is satisfied.

Definition 3 [Energy and data] Energy functionals for solutions and data are defined as follows:

$$
\begin{aligned}
E(\mathbf{u}(t)) \equiv & \frac{1}{2}\left[\|\nabla \cdot \mathbf{u}(t)\|^{2}+\left.\|\nabla \mathbf{u}\|\right|^{2}+\left(\nabla \mathbf{u}, \nabla \mathbf{u}^{T}\right)\right] \\
E(p(t))= & E_{\mathbf{u}}(p(t)) \equiv(k(\nabla \cdot \mathbf{u}) \nabla p, \nabla p) \\
\left.\operatorname{DATA}_{0}\right|_{0} ^{T} \equiv & \int_{0}^{T}\left[\|\mathbf{g}(t)\|_{L^{2}\left(\Gamma_{N}\right)}^{2}+\|\psi(t)\|_{L^{2}\left(\Gamma_{D, v}\right)}^{2}+\|S(t)\|_{L^{2}(\Omega)}^{2}+\|\mathbf{F}(t)\|_{L^{2}(\Omega)}^{2}\right. \\
& \left.+\left\|\mathbf{g}_{t}(t)\right\|_{L^{2}\left(\Gamma_{N}\right)}^{2}+\left\|\mathbf{F}_{t}(t)\right\|_{L^{2}(\Omega)}^{2}\right] d t+\sup _{[0, T]}\left[\|\mathbf{F}(t)\|^{2}+\|\mathbf{g}(t)\|_{L^{2}\left(I_{N}\right)}^{2}\right] \\
\left.\operatorname{DATA}_{\delta}\right|_{0} ^{T} \equiv & \int_{0}^{T}\left[\|\mathbf{g}(t)\|_{L^{2}\left(\Gamma_{N}\right)}^{2}+\|\psi(t)\|_{L^{2}\left(\Gamma_{D, v}\right)}^{2}+\|S(t)\|_{L^{2}(\Omega)}^{2}+\|\mathbf{F}(t)\|_{L^{2}(\Omega)}^{2}\right] d t
\end{aligned}
$$

Remark 2 The test functions of the form $\mathbf{w}(\mathbf{x}) f(t)$, with $\mathbf{w} \in \mathbf{V}$ and $f \in C_{0}^{\infty}((0, T))$, are dense in $L^{2}(0, T ; \mathbf{V})$; similarly, test functions of the form $q(\mathbf{x}) f(t)$, with $q \in V$ and $f \in C_{0}^{\infty}((0, T))$, are dense in $L^{2}(0, T ; V)$.

Remark 3 (Initial Conditions) When $\delta>0$, owing to the time dynamics in the elasticity equation (15), an initial condition on the displacement $\mathbf{u}^{0} \in \mathbf{V}$ is prescribed. Since the mass balance equation (16) requires a specification of $d_{0}=$ $\nabla \cdot \mathbf{u}(0) \in L^{2}(\Omega)$ for the fluid content $\zeta=\nabla \cdot \mathbf{u}$, we introduce a compatibility condition between $d_{0}$ and $\mathbf{u}^{0}$ requiring that $\nabla \cdot \mathbf{u}^{0}=d_{0}$. On the other hand, when $\delta=0$, the momentum equation does not involve any time derivative on the displacement and therefore only the initial condition $d_{0}=\nabla \cdot \mathbf{u}(0) \in L^{2}(\Omega)$ would suffice. However, in obtaining the a priori estimates for the solutions described below (i.e., in the process of constructing the solutions-see Lemma 7 and Lemma 10) it will be necessary to consider only those $d_{0} \in L^{2}(\Omega)$ such that there exists an $\mathbf{u}^{0} \in \mathbf{V}$ satisfying $\nabla \cdot \mathbf{u}^{0}=d_{0}$, since terms of the form $\left\|\mathbf{u}^{0}\right\|_{\mathbf{v}}$ appear on the right hand side of the estimates. This is in line with [55], though for the approach taken in [51] for weak solutions, it is enough to specify $d_{0}$ independently of any reference to a preimage in $\mathbf{V}$.

Remark 4 The notion of data in Definition 3 is fundamentally different depending on whether the parameter $\delta$ is strictly positive or is equal to zero. When $\delta>0$, the notion of time differentiability for the solution is stronger than in the case $\delta=0$, as shown by the fact that identities (25)-(26) include time derivatives of $\mathbf{u}$, whereas identities (27)-(28) do not. As a consequence, time regularity requirements on the data are significantly weaker in the case $\delta>0$ than in the case $\delta=0$, as shown by the comparison between (32) and (31).

Remark 5 Volumetric and boundary forcing terms analogous to our $\mathbf{F}, S, \mathbf{g}, \psi$ are also included in $[6,51,55]$. In [55], the author does not consider viscoelastic ef-

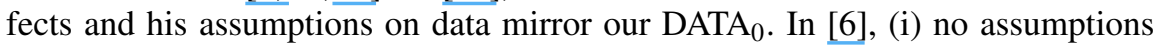


are placed directly on the body force $\mathbf{F}$, and (ii) homogeneous boundary conditions are imposed on pressure and displacement. We note that [6] appeals to [51] in dealing with $\mathbf{F}$ via a simple translation argument (see [51, p. 323-324]) and this argument is not applicable to the viscoelastic case $(\delta>0)$. Additionally, we emphasize that the regularity requirements mirror those of our DATA 0 when utilizing the translation described in [51] in order to obtain equivalence for well-posedness of the homogeneous and inhomogeneous cases of $\mathbf{F}$ and $\mathbf{g}$. Indeed, (i) the spatial and temporal regularity of $\mathbf{F}(t)$ must match that of $(-\mathscr{E})^{1 / 2}(\mathbf{u}(t))$ (where $\mathscr{E}$ is the elasticity operator introduced in Section 3.3 below), and, (ii) either $\nabla \cdot \mathbf{u}_{t}$ must be well defined in $L^{2}(\Omega)$, which does necessarily follow directly from the equations, or the boundary data $\mathbf{g}(t)$ must be differentiable in the sense of $H^{1}\left(0, T ; L^{2}\left(\Gamma_{N}\right)\right)$.

We now list our baseline assumptions on the domain, as well as the permeability function $k(\cdot)$ :

Assumption 3.1 We assume:

1. $\Gamma_{D}$ is a set of positive measure, so by Korn's inequality:

$$
E(\mathbf{u}(t)) \geq c\|\mathbf{u}(t)\|_{\left(H^{1}(\Omega)\right)^{3}}^{2} .
$$

2. $\Gamma_{D, p}$ is a set of positive measure, so by Poincare's inequality:

$$
\|v\|_{L^{2}(\Omega)} \leq C_{P}\|\nabla v\|_{L^{2}(\Omega)}, \forall v \in V .
$$

3. The scalar function $k(\cdot): \mathbb{R} \rightarrow \mathbb{R}$ is continuous on $\mathbb{R}$. We assume $k(s) \geq \kappa>0$ $\forall s \in \mathbb{R}$, so there is a constant $C(\kappa)$ so that:

$$
\|p\|_{1} \leq C(\kappa) E(p(t)) .
$$

Additionally, we assume: $k(s) \leq \hat{\kappa}<\infty \forall s \in \mathbb{R}$.

4. We assume the boundary $\Gamma$ is such that Lemma 2 holds. (See the following section and discussion.)

\subsection{Elasticity operator}

In the analysis of the momentum equation, we consider a given $p \in L^{2}(\Omega)$ (and thus $\nabla p \in \mathbf{V}^{\prime}$, with $\mathbf{V}^{\prime}$ denoting the dual space of $\mathbf{V}$ ) and produce a corresponding $\mathbf{u} \in \mathbf{V}$ which satisfies the stationary elasticity equation. Define $\mathscr{E}: \mathbf{V} \rightarrow \mathbf{V}^{\prime}$ to be the elasticity operator given by

$$
\mathscr{E}(\mathbf{u})=-\nabla \cdot(2 \varepsilon(\mathbf{u})+(\nabla \cdot \mathbf{u}) \mathbf{I}), \forall \mathbf{u} \in\left(C_{0}^{\infty}(\Omega)\right)^{3}
$$

with domain

$$
\mathscr{D}(\mathscr{E}) \equiv\left\{\mathbf{u} \in \mathbf{V}: \nabla \cdot(2 \varepsilon(\mathbf{u})+(\nabla \cdot \mathbf{u}) \mathbf{I}) \in L^{2}(\Omega)\right\} .
$$

Note that the boundary conditions for the operator $\mathscr{E}$ are built into the space $\mathbf{V}$; the operator $\mathscr{E}$ is specified by the bilinear form $a(\cdot, \cdot)$ on $\mathbf{V} \times \mathbf{V}$ as given by (24).

We remark that we can also write

$$
\mathscr{E}(\mathbf{u})=-\nabla \cdot \mathbf{T}_{e}(\mathbf{u}), \forall \mathbf{u} \in\left(C_{0}^{\infty}(\Omega)\right)^{3},
$$


where the purely elastic stress $\mathbf{T}_{e}(\mathbf{u})$ has been defined in (5), here including elastic constant normalized to unity for the purpose of simplifying the exposition of the theoretical analysis. It is known that $\mathscr{E}: \mathbf{V} \rightarrow \mathbf{V}^{\prime}$ is an isomorphism [12,51]. This resulting lemma is classical (see e.g., [12,29] and references therein), but in this functional setup we directly cite [51]:

Lemma 1 Given $p \in V$ (so $\left.\left.p\right|_{\Gamma_{N}} \in L^{2}\left(\Gamma_{N}\right)\right), \mathbf{g} \in\left(L^{2}\left(\Gamma_{N}\right)\right)^{3}$, and $\mathbf{F} \in\left(L^{2}(\Omega)\right)^{3}$, the elasticity problem

$$
\begin{cases}-\nabla \cdot(2 \varepsilon(\mathbf{u})+(\nabla \cdot \mathbf{u}) \mathbf{I})=-\nabla p-\mathbf{F} & \in \mathbf{V}^{\prime} \\ \mathbf{u}=\mathbf{0} & \text { on } \Gamma_{D} \\ \mathbf{T}_{e}(\mathbf{u}) \mathbf{n}=\mathbf{g}+\left.p\right|_{\Gamma_{N}} \mathbf{n} & \text { on } \Gamma_{N}\end{cases}
$$

is well-posed with a solution $\mathbf{u} \in \mathbf{V}$.

Mixed-type boundary conditions for the elasticity operator $\mathscr{E}$ are important for many applications.

Remark 6 In [6] the boundary is composed of a single Dirichlet (clamped) component upon which both pressure $p$ and displacement $\mathbf{u}$ are zero. In this case, or when $\overline{\Gamma_{N}} \cap \overline{\Gamma_{D}}=\emptyset$ (see [51]), elliptic theory recovers full $\left(H^{2}(\Omega)\right)^{3} \cap \mathbf{V}$ regularity of the displacement $\mathbf{u}$ when $p \in V$; from this, $\nabla \cdot \mathbf{u} \in H^{1}(\Omega)$, which is used freely in [6].

Unlike in [6] (and to a certain extent [51]), we will not obtain full $\left(H^{2}(\Omega)\right)^{3}$ regularity of the solution $\mathbf{u}$ accompanying (33). However, some elliptic regularity is recovered:

1. In [39, p.347], for the Lamé system in polyhedral domains, given $p \in V$, $\mathbf{F} \in\left(L^{2}(\Omega)\right)^{3}$, and $\mathbf{g} \in\left(H^{1 / 2}\left(\Gamma_{N}\right)\right)^{3}$ and under certain geometrical assumptions on the boundary $\Gamma$ (in particular that $\Gamma_{N}$ and $\Gamma_{D}$ do not meet tangentially and the supremum of their dihedral angles is strictly less than $\pi$, one obtains the regularity $\mathbf{u} \in\left(H^{3 / 2+\varepsilon}(\Omega)\right)^{3}$ for the displacement (an analogous result is obtained for 2-D polygonal domains [39]).

2. Additionally, in the limiting case, when one only assumes that $\Gamma$ is $C^{1,1}$, the results from [49] provide $H^{3 / 2-\varepsilon}(\Omega)$ regularity of solutions for scalar elliptic problems (with analogous assumptions on the data; in fact, the Neumann data can be taken only in $H^{-\varepsilon}(\Omega)$ ).

3. Other regularity theorems for the Lamé system with mixed boundary conditions are available in [37,47] (and references therein), for instance, in weighted Sobolev spaces.

Remark 7 In [39], geometrical assumptions provide maximal elliptic regularity (for data analogous to what is considered herein), that is, a $3 / 2+\varepsilon$ (Sobolev exponent). In the more general case of [49] (for scalar elliptic equations with appropriately regular data) elliptic regularity is recovered up to (not including) $3 / 2$. Such a result should also hold for the Lamé system. In the construction of solutions below, we only utilize elliptic regularity of $\left(H^{1+\varepsilon}(\Omega)\right)^{3}$ corresponding to solutions of (33). 
In the analyses $[6,51]$ the authors utilize a map $B$ which takes as input pressure information $p$ and gives $\nabla \cdot \mathbf{u}$ as output. Clearly the boundary conditions are an issue here. In [51] this $B$ map is defined on a direct sum space which incorporates boundary conditions (and allows for lower regularity of $p$ ); in [6] homogenous boundary conditions are considered to drastically simplify the analysis.

Here we analyze the problem in our setup, i.e., with mixed boundary conditions. We can consider a continuous map $B: V \rightarrow L^{2}(\Omega)$ such that

$$
B p=\nabla \cdot \mathbf{u}
$$

where $\mathbf{u}$ is the solution to (33). Based on the discussion above, we have:

Lemma 2 Given $p \in V$ the corresponding elliptic solver $\mathscr{E}^{-1}(-\nabla p+\mathbf{F})=\mathbf{u}$ lies in $\left(H^{1+\varepsilon}(\Omega)\right)^{3} \cap \mathbf{V}$ for some $\varepsilon>0$ (depending on the domain) with associated bound. Thus, we have $B p=\nabla \cdot \mathbf{u} \in H^{\varepsilon}(\Omega)$, which yields that

$$
B: V \rightarrow H^{\varepsilon}(\Omega) \text {, continuously. }
$$

Remark 8 This fact will be critical to invoke compactness results when passing to the limit in time on approximate solutions below.

There are additional properties of the $B$ operator (reminiscent of [51]) that we will take advantage of. The $B$ mapping is injective:

Lemma 3 For $p \in V$, if $B p=0$ (in the sense of $L^{2}(\Omega)$ ), then $p=0$.

Proof Suppose that $B p=0$ in $L^{2}(\Omega)$. Then $(B p, q)=0$ for all $q \in H_{0}^{1}(\Omega)$. But then $(\mathbf{u}, \nabla q)=0$ for all such $q$. This means that

$$
\left(\mathscr{E}^{-1}(\nabla p), \nabla q\right)=0
$$

for all $q \in H_{0}^{1}(\Omega)$. From this we infer that $\mathscr{E}^{-1}(\nabla p)=0 \in L^{2}(\Omega)$. Since the elasticity problem is well-posed, we have that $\nabla p=\mathbf{0}$. However, owing to the fact that $p \in V$, we must have that $p \equiv 0$. Thus $\operatorname{Ker}(B)=\{0\}$.

We also have the following additional result, which we state as a lemma (see [51, pp. 325-326]):

Lemma 4 Suppose that $p_{n} \in V$ (so the trace $\left.p\right|_{\Gamma}$ is defined) with $p_{n} \rightarrow p$ in $L^{2}(\Omega)$ (e.g., if $p_{n} \rightarrow p$ in $\left.V\right)$. Then $B p_{n} \rightarrow B p$ in $L^{2}(\Omega)$.

Remark 9 In considering limit passage of time-discretized approximate solutions we will not obviously have the analogous result; namely, if $p_{\Delta t} \rightarrow p \in L^{2}\left(0, T ; L^{2}(\Omega)\right)$ $B p_{\Delta t}$ does not necessarily converge strongly to $B p$ in the same sense. The presence of viscoelastic terms does not change this fact. Aubin's compactness criteria must be invoked, which requires Lemma 2. 


\subsection{Existence of solutions: main theorems}

To show existence of solutions to both (25)-(26) and (27)-(28) we follow these steps:

1. We introduce approximate problems corresponding to spatial and temporal discretizations in both cases $\delta=0$ and $\delta>0$. We again follow the approach presented in [55].

2. We adapt a technique described in [6] for solving a weak form of the fully discretized versions of (25)-(26) and (27)-(28).

3. A priori estimates are obtained for the discretized solutions (and later for the solutions themselves in Section 3.5), i.e., a priori bounds on the approximate solutions which are uniform with respect to the discretization parameters.

4. We then utilize compactness results to extract limit points which solve the weak form of the appropriate equations.

Remark 10 Various approximation techniques have been proposed to study the well-posedeness of PDE systems similar to that in (15)-(22). In [40], a method based on the Galerkin approximation is utilized. However, the physical boundary conditions we consider prevent us from easily solving the ODE system associated with (15)-(22), despite the fact that, at least for $\delta>0$, we obtain sufficient regularity for $\mathbf{u}_{t}$ (i.e., $\mathbf{u}$ is differentiable in time into $\left.\left(L^{2}(\Omega)\right)^{3}\right)$. A discrete-based approach is also presented in [6], but it is greatly streamlined and simplified via the homogeneous Dirichlet boundary conditions for both pressure and displacement. In our work, we follow closely the discrete-based approach presented in [55], where the model in (15)-(22) is considered under the assumption of constant permeability, and we utilize techniques from [6] to treat the nonlinearity arising from the fact that $k=k(\nabla \cdot \mathbf{u})$.

3.4.1 The viscoelastic case: $\delta>0$

Theorem 1 [Existence of VE-Solutions] Consider (15)-(22) with $\delta>0$. Let Assumption 3.1 hold, and consider data of the form:

$$
\begin{array}{cl}
\mathbf{F} \in L^{2}\left(0, T ;\left(L^{2}(\Omega)\right)^{3}\right), & S \in L^{2}\left(0, T ; L^{2}(\Omega)\right), \\
\mathbf{g} \in L^{2}\left(0, T ;\left(H^{1 / 2}\left(\Gamma_{N}\right)\right)^{3}\right), & \psi \in L^{2}\left(0, T ; L^{2}\left(\Gamma_{D, v}\right)\right) .
\end{array}
$$

Then, there exists a VE-solution (in the sense of (25)-(26)) satisfying

$$
\begin{aligned}
\sup _{t \in[0, T]} E(\mathbf{u}(t))+\int_{0}^{T} & {\left[E(p(t))+E(\mathbf{u}(t))+E\left(\mathbf{u}_{t}\right)\right] d t } \\
\leq & C_{1}\left[E(\mathbf{u}(0))+\left.\left(\frac{1}{1+\delta}\right) D A T A_{\delta}\right|_{0} ^{T}\right] \exp \left(\frac{C_{2} T}{1+\delta}\right) .
\end{aligned}
$$

\section{Step 1: The discretized problem}

Let us partition the time interval $[0, T]$ into $r$ sub-intervals, yielding $\Delta t=T / r$ and $t_{i}=i \Delta t, i=0,1, \ldots, r$. We will solve the problem iteratively, i.e., by solving an 
approximate problem on $\left(t_{i-1}, t_{i}\right]$ we will have data to feed into the problem on $\left(t_{i}, t_{i+1}\right]$. Define $\mathbf{g}^{i}$ by

$$
\mathbf{g}^{i} \equiv \frac{1}{\Delta t} \int_{t_{i-1}}^{t_{i}} \mathbf{g}(\mathbf{x}, t) d t
$$

with $\mathbf{F}^{i}, \psi^{i}, S^{i}$ defined analogously. We now define a (time-scaled) weak form of the temporal semi-discretized problem when $\delta>0$ as:

$$
\begin{aligned}
& \delta a\left(\mathbf{u}^{i}-\mathbf{u}^{i-1}, \mathbf{w}\right)+[\Delta t] a\left(\mathbf{u}^{i}, \mathbf{w}\right)-[\Delta t]\left(p^{i}, \nabla \cdot \mathbf{w}\right) \\
&= {[\Delta t]\left\langle\mathbf{g}^{i}, \mathbf{w}\right\rangle_{\Gamma_{N}}-[\Delta t]\left(\mathbf{F}^{i}, \mathbf{w}\right) } \\
& {[\Delta t]^{2}\left(k\left(\nabla \cdot \mathbf{u}^{i}\right) \nabla p^{i}, \nabla q\right) }+[\Delta t]\left(\nabla \cdot \mathbf{u}^{i}-\nabla \cdot \mathbf{u}^{i-1}, q\right) \\
&=-[\Delta t]^{2}\left\langle\psi^{i}, q\right\rangle_{\Gamma_{D, v}}+[\Delta t]^{2}\left(S^{i}, q\right) \\
& \mathbf{u}(0)=\mathbf{u}^{0}, \quad \nabla \cdot \mathbf{u}^{0}=d_{0}
\end{aligned}
$$

This means that for all $(\mathbf{w}, q) \in \mathbf{V} \times V$, we have

$$
\begin{aligned}
(\delta+\Delta t) a\left(\mathbf{u}^{i}, \mathbf{w}\right) & -[\Delta t]\left(p^{i}, \nabla \cdot \mathbf{w}\right) \\
= & \delta a\left(\mathbf{u}^{i-1}, \mathbf{w}\right)+[\Delta t]\left\langle\mathbf{g}^{i}, \mathbf{w}\right\rangle_{\Gamma_{N}}-[\Delta t]\left(\mathbf{F}^{i}, \mathbf{w}\right) \\
{[\Delta t]^{2}\left(k\left(\nabla \cdot \mathbf{u}^{i}\right) \nabla p^{i}, \nabla q\right) } & +[\Delta t]\left(\nabla \cdot \mathbf{u}^{i}, q\right) \\
= & {[\Delta t]\left(\nabla \cdot \mathbf{u}^{i-1}, q\right)-[\Delta t]^{2}\left\langle\psi^{i}, q\right\rangle_{\Gamma_{D, v}}+[\Delta t]^{2}\left(S^{i}, q\right), }
\end{aligned}
$$

along with the initial conditions (38). The problem in (39) and (40) is further discretized in space. To this end, let us consider a basis $B$ for $V$ and a basis $\mathbf{B}$ for $\mathbf{V}$, and let $V_{h}$ and $\mathbf{V}_{h}$ denote finite dimensional subspaces of $V$ and $\mathbf{V}$ corresponding to finite spans in $B$ and $\mathbf{B}$, respectively, each parametrized by a parameter $h$. We also set $\mathbb{V}_{h}=V_{h} \times \mathbf{V}_{h}$.

We will consider (36)-(38) on $\mathbb{V}_{h}$; that is, with $\mathbf{u}_{h}^{i-1}(i=2, . . r)$ as given data from the previous iteration, we can solve (39)-(40) on $\mathbb{V}_{h}$ yielding $\left(\mathbf{u}_{h}^{i}, p_{h}^{i}\right)$. The initial condition $\mathbf{u}_{h}^{0}$ is obtained as the projection of $\mathbf{u}^{0} \in \mathbf{V}$ onto $\mathbf{V}_{h}$, with its divergence providing $d_{0, h}$ via the compatibility condition. We note that $\left(\mathbf{u}_{h}^{1}, p_{h}^{1}\right)$ are obtained by solving (36)-(38) on $\mathbb{V}_{h}$ with $\mathbf{u}^{0}, \mathbf{g}^{0}, \mathbf{F}^{0}, \psi^{0}$, and $S^{0}$ given as datathis is to say $p^{0}$ is not explicitly solved for in this scheme.

\section{Step 2: Solving the fully discretized problem}

In [55], the assumption of constant permeability allows for a straightforward approach to the algebraic equations arising from the weak form of the discretized problem. Here, owing to the nonlinearity $k=k(\nabla \cdot \mathbf{u})$, the solution of (36)-(38) is nontrivial. We utilize a fixed point method, as in [6]. Given $\left(\mathbf{u}^{i-1}, p^{i-1}, \psi^{i}, \mathbf{g}^{i}, \mathbf{F}^{i}, S^{i}\right)$, the problem consists in finding $\left(p_{h}^{i}, \mathbf{u}_{h}^{i}\right) \in \mathbb{V}_{h}$ satisfying (36)-(38). More precisely:

Lemma 5 Consider data of the form $\left(\mathbf{u}^{i-1}, p^{i-1}, \psi^{i}, \mathbf{g}^{i}, \mathbf{F}^{i}, S^{i}\right)$-with $\left(p^{i-1}, \mathbf{u}^{i-1}\right) \in$ $\mathbb{V}_{h}$ (with the understanding that at time $t=0$, we take the projection of $\mathbf{u}^{0} \in \mathbf{V}$ onto $\left.\mathbf{V}_{h}\right)$. Then there is a solution $\left(\bar{p}_{h}^{i}, \overline{\mathbf{u}}_{h}^{i}\right) \in \mathbb{V}_{h}$ which satisfies (36)-(38) for all test functions $(\mathbf{w}, q) \in \mathbb{V}_{h}$. 
Proof (of Lemma 5) We define a map $G_{\delta}: \mathbb{V}_{h} \rightarrow \mathbb{V}_{h}$ by the bilinear form below (corresponding to the weak form of the discretized problem): for $\left(p^{i}, \mathbf{u}^{i}\right) \in \mathbb{V}_{h}$

$$
\begin{aligned}
\left(G_{\delta}\left[\begin{array}{c}
p^{i} \\
\mathbf{u}^{i}
\end{array}\right],\left[\begin{array}{c}
q \\
\mathbf{w}
\end{array}\right]\right)= & \delta+\Delta t) a\left(\mathbf{u}^{i}, \mathbf{w}\right)+[\Delta t]\left(\nabla \cdot \mathbf{u}^{i}, q\right) \\
& -[\Delta t]\left(p^{i}, \nabla \cdot \mathbf{w}\right)+[\Delta t]^{2}\left(k\left(\nabla \cdot \mathbf{u}^{i}\right) \nabla p^{i}, \nabla q\right) \\
& -\delta a\left(\mathbf{u}^{i-1}, \mathbf{w}\right)-[\Delta t]\left(\nabla \cdot \mathbf{u}^{i-1}, q\right) \\
& -[\Delta t]\left\langle\mathbf{g}^{i}, \mathbf{w}\right\rangle_{\Gamma_{N}}+[\Delta t]\left(\mathbf{F}^{i}, \mathbf{w}\right)+[\Delta t]^{2}\left\langle\psi^{i}, q\right\rangle_{\Gamma_{D, v}}-[\Delta t]^{2}\left(S^{i}, q\right)
\end{aligned}
$$

for all $(q, \mathbf{w}) \in \mathbb{V}_{h}$. (Note: $G_{\delta}$ defines an operator on the whole $\mathbb{V}_{h}$, namely on $\mathbf{V}_{h}$ and $V_{h}$ simultaneously.) We now note the following:

$$
\begin{aligned}
\left(G_{\delta}\left[\begin{array}{c}
p^{i} \\
\mathbf{u}^{i}
\end{array}\right],\left[\begin{array}{c}
p^{i} \\
\mathbf{u}^{i}
\end{array}\right]\right)= & (\delta+\Delta t) 2 E\left(\mathbf{u}^{i}\right)+[\Delta t]^{2} E\left(p^{i}\right) \\
& -\delta a\left(\mathbf{u}^{i-1}, \mathbf{u}^{i}\right)-[\Delta t]\left(\nabla \cdot \mathbf{u}^{i-1}, p^{i}\right) \\
& -[\Delta t]\left\langle\mathbf{g}^{i}, \mathbf{u}^{i}\right\rangle_{\Gamma_{N}}+[\Delta t]\left(\mathbf{F}^{i}, \mathbf{u}^{i}\right) \\
& +[\Delta t]^{2}\left\langle\psi^{i}, p^{i}\right\rangle_{\Gamma_{D, v}}-[\Delta t]^{2}\left(S^{i}, p^{i}\right)
\end{aligned}
$$

from which it follows that:

$$
\begin{aligned}
\left(G_{\delta}\left[\begin{array}{c}
p^{i} \\
\mathbf{u}^{i}
\end{array}\right],\left[\begin{array}{c}
p^{i} \\
\mathbf{u}^{i}
\end{array}\right]\right) \geq & C_{1}\left[(\delta+\Delta t)\left\|\mathbf{u}^{i}\right\|_{1}^{2}+\kappa[\Delta t]^{2}\left\|p^{i}\right\|_{1}^{2}\right] \\
& -C_{2}\left[[\Delta t]\left\|\mathbf{u}^{i-1}\right\|_{1}+[\Delta t]^{2}\left\|\psi^{i}\right\|_{L^{2}\left(\Gamma_{D, v}\right)}+[\Delta t]^{2}\left\|S^{i}\right\|_{L^{2}(\Omega)}\right]\left\|p^{i}\right\|_{1} \\
& -C_{3}\left[\delta\left\|\mathbf{u}^{i-1}\right\|_{1}+[\Delta t]\left\|\mathbf{g}^{i}\right\|_{L^{2}\left(\Gamma_{N}\right)}+[\Delta t]\left\|\mathbf{F}^{i}\right\|_{L^{2}(\Omega)}\right]\left\|\mathbf{u}^{i}\right\|_{1} .
\end{aligned}
$$

We have used Korn's lemma, Poincare's inequality, the trace theorem, and the lower bound on $k(\cdot)$ (embodied in the constants $C_{i}$ and $\kappa$ ). Finally, using Young's inequality, taking $\Delta t$ sufficiently small (depending on $\delta$ ), we have:

$$
\begin{aligned}
\left(G_{\delta}\left[\begin{array}{l}
p^{i} \\
\mathbf{u}^{i}
\end{array}\right],\left[\begin{array}{l}
p^{i} \\
\mathbf{u}^{i}
\end{array}\right]\right) \geq & C(\delta, \kappa) \cdot[\Delta t]^{2}\left\|\left[\begin{array}{l}
p^{i} \\
\mathbf{u}^{i}
\end{array}\right]\right\|_{\mathbb{V}_{h}}^{2} \\
& -c \cdot[\Delta t]\left[\left\|\mathbf{g}^{i}\right\|_{L^{2}\left(\Gamma_{N}\right)}^{2}+\left\|\mathbf{F}^{i}\right\|_{0}^{2}+\left\|\mathbf{u}^{i-1}\right\|_{1}^{2}\right] \\
& -c \cdot[\Delta t]^{2}\left[\left\|\psi^{i}\right\|_{L^{2}\left(\Gamma_{D, v}\right)}^{2}+\left\|S^{i}\right\|_{0}^{2}\right] .
\end{aligned}
$$

Thus the mapping $G_{\delta}: \mathbb{V}_{h} \rightarrow \mathbb{V}_{h}$ has the property that

$$
\left(G_{\delta}\left[\begin{array}{l}
p^{i} \\
\mathbf{u}^{i}
\end{array}\right],\left[\begin{array}{l}
p^{i} \\
\mathbf{u}^{i}
\end{array}\right]\right) \geq 0
$$

when

$$
\left\|\left[\begin{array}{l}
p^{i} \\
\mathbf{u}^{i}
\end{array}\right]\right\|_{\mathbb{V}_{h}}^{2} \geq \frac{c\left[\left\|\mathbf{g}^{i}\right\|_{L^{2}\left(\Gamma_{N}\right)}^{2}+\left\|\mathbf{F}^{i}\right\|_{0}^{2}+\left\|\mathbf{u}^{i-1}\right\|_{1}^{2}+[\Delta t]\left(\left\|\psi^{i}\right\|_{L^{2}\left(\Gamma_{D, v}\right)}^{2}+\left\|S^{i}\right\|_{0}^{2}\right)\right]}{C(\boldsymbol{\delta}, \kappa)[\Delta t]} .
$$


Continuity of $G_{\delta}$ on $\mathbb{V}_{h}$ follows straightforwardly from the definition (41) and from the uniform upper bound on the permeability $k(s) \leq \hat{\kappa}$. With these two properties of $G_{\delta}$ established on $\mathbb{V}_{h}$ (noting that $\mathbb{V}_{h}$ is finite dimensional), we may utilize a corollary to Brouwer's fixed point theorem [53, Corollary A.12.], which guarantees that there exists a point $\left(\bar{p}^{i}, \overline{\mathbf{u}}^{i}\right) \in \mathbb{V}_{h}$ satisfying:

$$
\left(G_{\delta}\left[\begin{array}{c}
\bar{p}^{i} \\
\overline{\mathbf{u}}^{i}
\end{array}\right],\left[\begin{array}{c}
q \\
\mathbf{w}
\end{array}\right]\right)=0
$$

for all $(q, \mathbf{w}) \in \mathbb{V}_{h}$. Moreover, $\left(\bar{p}^{i}, \overline{\mathbf{u}}^{i}\right)$ has the property that:

$$
\left\|\left[\begin{array}{c}
\bar{p}^{i} \\
\overline{\mathbf{u}}^{i}
\end{array}\right]\right\|_{\mathbb{V}_{h}}^{2} \leq \frac{c\left[\left\|\mathbf{g}^{i}\right\|_{L^{2}\left(\Gamma_{N}\right)}^{2}+\left\|\mathbf{F}^{i}\right\|_{0}^{2}+\left\|\mathbf{u}^{i-1}\right\|_{1}^{2}+[\Delta t]\left(\left\|\psi^{i}\right\|_{L^{2}\left(\Gamma_{D, v}\right)}^{2}+\left\|S^{i}\right\|_{0}^{2}\right)\right]}{C(\boldsymbol{\delta}, \kappa)[\Delta t]} .
$$

We have thus produced a weak solution of the discretized problem (36)-(38) on $\mathbb{V}_{h}$ (for each $i, i=1, \ldots, r$ ) from the data given at the previous iterate $i-1$. Moreover, this solution enjoys an a priori bound via (45).

Remark 11 The estimate in (45) appears singular as $\Delta t \rightarrow 0$. However, we need the fixed point argument only to guarantee the existence of a solution to the (nonlinear) finite dimensional discretized problem (36)-(38). We will appeal to other a priori estimates obtained via direct "multiplier" methods (see Lemma 7, and Lemma 10).

We note that the above result holds for any $h>0$, and hence we label the solutions

$$
\left(\bar{p}_{h}^{i}, \overline{\mathbf{u}}_{h}^{i}\right) \in \mathbb{V}_{h} \subset V \times \mathbf{V}
$$

with (45) providing a uniform bound in $h(>0)$; namely,

$$
\left\|\left[\begin{array}{c}
\bar{p}_{h}^{i} \\
\overline{\mathbf{u}}_{h}^{i}
\end{array}\right]\right\|_{V \times \mathbf{V}} \leq C_{i-1}(\delta, \kappa, \Delta t)
$$

where the subscript $i-1$ denotes dependence on the solution and data from the previous time step.

\section{Step 3: Limit passage in space}

Lemma 6 Consider data of the form $\left(\mathbf{u}^{i-1}, p^{i-1}, \psi^{i}, \mathbf{g}^{i}, \mathbf{F}^{i}, S^{i}\right)$-with $\left(p^{i-1}, \mathbf{u}^{i-1}\right) \in$ $\mathbb{V}$. Then there is a solution $\left(\bar{p}^{i}, \overline{\mathbf{u}}^{i}\right) \in V \times \mathbf{V}$ which satisfies (36)-(38) for all test functions $(\mathbf{w}, q) \in \mathbb{V}$.

Proof (of Lemma 6) From the uniform bound in the previous section we extract a subsequence (maintaining the same label) and weak limit point $\left(\bar{p}^{i}, \overline{\mathbf{u}}^{i}\right) \in V \times \mathbf{V}$ such that

$$
\left(\bar{p}_{h}^{i}, \overline{\mathbf{u}}_{h}^{i}\right) \rightarrow\left(\bar{p}^{i}, \overline{\mathbf{u}}^{i}\right) \text { in } V \times \mathbf{V} .
$$

We now proceed to show that these limit points are solutions to (39)-(40) considered on the entire space $V \times \mathbf{V}$, that is, for all test functions $q \in V$ and $\mathbf{w} \in \mathbf{V}$. We proceed to pass to the limit in (40). First, since $\overline{\mathbf{u}}_{h}^{i} \rightarrow \overline{\mathbf{u}}^{i}$ in $\mathbf{V} \equiv\left(H_{\Gamma_{D}}^{1}\right)^{3}$, we have that $\nabla \cdot \overline{\mathbf{u}}_{h}^{i} \rightarrow \nabla \cdot \overline{\mathbf{u}}^{i}$ in $L^{2}(\Omega)$. Therefore $\left|\left(\nabla \cdot \overline{\mathbf{u}}_{h}^{i}, q\right)-\left(\nabla \cdot \overline{\mathbf{u}}^{i}, q\right)\right| \rightarrow 0$ forall 
$q \in V$. Secondly, we deal with the nonlinear term $\left(k\left(\nabla \cdot \overline{\mathbf{u}}_{h}^{i}\right) \nabla \bar{p}_{h}^{i}, \nabla q\right)$. Noting that $\bar{p}_{h}^{i} \rightarrow \bar{p}^{i}$ in $V$, then, due to the compactness of the Sobolev embedding $V \hookrightarrow L^{2}(\Omega)$, we have that

$$
\bar{p}_{h}^{i} \rightarrow \bar{p}^{i} \text { in } L^{2}(\Omega) .
$$

We now show that $\nabla \cdot \overline{\mathbf{u}}_{h}^{i} \rightarrow \nabla \cdot \overline{\mathbf{u}}^{i}$ in $L^{2}(\Omega)$. Indeed, recalling that $\mathbf{u}^{i-1} \in \mathbf{V}$ is given as data, let $\mathbf{u}^{i, *} \in \mathbf{V}$ be the corresponding elasticity solution for $\bar{p}^{i}$, i.e., $\mathbf{u}^{i, *}$ solves (in the weak sense):

$$
\begin{cases}-(\boldsymbol{\delta}+\Delta t) \mathscr{E}\left(\mathbf{u}^{i, *}\right)=-[\Delta t] \nabla \bar{p}^{i}-\delta \mathscr{E}\left(\mathbf{u}^{i-1}\right)-[\Delta t] \mathbf{F}^{i} & \Omega \\ \mathbf{u}^{i, *}=0 & \Gamma_{D} \\ \mathbf{T}\left(\mathbf{u}^{i, *}\right) \mathbf{n}=[\Delta t] \mathbf{g}^{i}+[\Delta t]\left(\bar{p}^{i}\right) \mathbf{n} & \Gamma_{N}\end{cases}
$$

We also denote $\mathbf{u}_{h}^{i, *} \in \mathbf{V}$ as the solution to (46) corresponding to $\nabla \bar{p}_{h}^{i}$, with $\bar{p}_{h}^{i} \in$ $V_{h}$. We note that by Lemma 4 we have that $\nabla \cdot \mathbf{u}_{h}^{i, *} \rightarrow \nabla \cdot \mathbf{u}^{i, *}$ in $L^{2}(\Omega)$ (since $\bar{p}_{h}^{i} \rightarrow \bar{p}^{i}$ in $L^{2}(\Omega)$ ). Moreover, by considering the weak form of (46) (given $\bar{p}_{h}^{i}$ ) on $\mathbf{V}_{h}$ providing the solution $\mathbf{u}_{h}^{i, *}$, and recalling that $\overline{\mathbf{u}}_{h}^{i}$ also satisfies (39) with test functions from $\mathbf{V}_{h}$, we see that $\mathbf{u}_{h}^{i, *}=\overline{\mathbf{u}}_{h}^{i}$ (in the same sense) for all $h$. Since $\nabla \cdot \overline{\mathbf{u}}_{h}^{i} \rightarrow \nabla \cdot \overline{\mathbf{u}}^{i}$, and $\nabla \cdot \overline{\mathbf{u}}_{h}^{i}=\nabla \cdot \mathbf{u}_{h}^{i, *} \rightarrow \nabla \cdot \mathbf{u}^{i, *}$ in $L^{2}(\Omega)$, then $\nabla \cdot \mathbf{u}^{i, *}=\nabla \cdot \overline{\mathbf{u}}^{i} \in$ $L^{2}(\Omega)$ and $\nabla \cdot \overline{\mathbf{u}}_{h}^{i} \rightarrow \nabla \cdot \overline{\mathbf{u}}^{i}$, as desired.

By the bounds $0<\kappa \leq k(s) \leq \widehat{\kappa}$ for all $s \in \mathbb{R}$, as well as the continuity of $k(\cdot)$, we may utilize the fact that $k(\cdot): L^{2}(\Omega) \rightarrow L^{2}(\Omega)$ is a Nemytskii operator [50]. Thus $k\left(\nabla \cdot \overline{\mathbf{u}}_{h}^{i}\right) \rightarrow k\left(\nabla \cdot \overline{\mathbf{u}}^{i}\right)$.

We choose a test function $q \in V \cap W^{1, \infty}(\Omega)$ and consider:

$$
\begin{aligned}
\mid\left(k\left(\nabla \cdot \overline{\mathbf{u}}^{i}\right) \nabla \bar{p}^{i}, \nabla q\right)- & \left(k\left(\nabla \cdot \overline{\mathbf{u}}_{h}^{i}\right) \nabla \bar{p}_{h}^{i}, \nabla q\right) \mid \\
= & \left|\left(k\left(\nabla \cdot \overline{\mathbf{u}}^{i}\right) \nabla\left[\bar{p}^{i}-\bar{p}_{h}^{i}\right], \nabla q\right)-\left(\left[k\left(\nabla \cdot \overline{\mathbf{u}}_{h}^{i}\right)-k\left(\nabla \cdot \overline{\mathbf{u}}^{i}\right)\right] \nabla \bar{p}_{h}^{i}, \nabla q\right)\right| \\
\leq & \widehat{\kappa}\left|\left(\nabla\left[\bar{p}^{i}-\bar{p}_{h}^{i}\right], \nabla q\right)\right|+\left.|| \nabla q\right|_{L^{\infty}(\Omega)}|| \nabla \bar{p}_{h}^{i}|| \cdot\left|k\left(\nabla \cdot \overline{\mathbf{u}}^{i}\right)-k\left(\nabla \cdot \overline{\mathbf{u}}_{h}^{i}\right)\right| \\
& \rightarrow 0,
\end{aligned}
$$

since $\bar{p}_{h}^{i} \rightarrow \bar{p}^{i}$ in $V$ and $k\left(\nabla \cdot \overline{\mathbf{u}}_{h}^{i}\right) \rightarrow k\left(\nabla \cdot \overline{\mathbf{u}}^{i}\right)$. By the density of $V \cap W^{1, \infty}(\Omega)$ in $V$, we have shown that the weak limit points $\left(\bar{p}^{i}, \overline{\mathbf{u}}^{i}\right)$ satisfy (39)-(40) for all $(q, \mathbf{w}) \in V \times \mathbf{V}$ with given data $\left(p^{i-1}, \mathbf{u}^{i-1}\right) \in \mathbb{V}$ and $\mathbf{g}^{i}, \mathbf{F}^{i}, \psi^{i}$ and $S^{i}$.

In this way, we can iteratively define a solution $\left\{\left(\bar{p}^{i}, \overline{\mathbf{u}}^{i}\right)\right\}_{i=1}^{r}$ for all discrete time levels $t_{i}=i \Delta t$. We must now pass to the limit in time, that is considering $\Delta t \rightarrow 0$.

\section{Step 4: Limit passage in time}

The key step for obtaining solutions is the following set of upper bounds that are uniform in $r$. 
Lemma 7 For each $i=1, \ldots, r$ solutions to (36)-(38) on $V \times \mathbf{V}$ enjoy the estimates

$$
\begin{array}{r}
{[\Delta t] \sum_{i=1}^{r}\left\|\bar{p}^{i}\right\|_{1}^{2} \leq C} \\
\left\|\overline{\mathbf{u}}^{i}\right\|_{1}^{2} \leq C \\
{[\Delta t] \sum_{i=1}^{r}\left\|\overline{\mathbf{u}}^{i}\right\|_{1}^{2} \leq C} \\
{[\Delta t] \sum_{i=1}^{r}\left\|\frac{\overline{\mathbf{u}}^{i}-\overline{\mathbf{u}}^{i-1}}{\Delta t}\right\|_{1}^{2} \leq C}
\end{array}
$$

where the constant $C$ above depends on $T, E\left(\mathbf{u}^{0}\right)$, and DATA $\left.\right|_{0} ^{T}($ see below (121)).

Proof (of Lemma 7) The proof of estimates (47)-(50) relies upon the utilization of the discrete test functions $p^{i}$ and $\left[\mathbf{u}^{i}-\mathbf{u}^{i-1}\right]$ in (39)-(40). We explicitly show these calculations in Lemma 10 corresponding to the analogous Step 4 for the more delicate $\delta=0$ case.

Remark 12 For $\delta>0$, a priori estimates may also be obtained in the continuous setting, as shown in Section 3.5. This has the advantage of producing so-called "energy identities" as intermediate points in the estimation. Note that, in contrast, for $\delta=0$ one only obtains energy inequalities by limit passage on discrete estimates (as below).

Remark 13 The final uniform estimate (50) on the difference quotient is one of the ways in which the $\delta>0$ case distinguishes from the $\delta=0$ case, where no such estimate holds. Having this bound provides additional regularity for the velocity $\mathbf{u}_{t}$, even though one must associate the weak limit of the difference quotients with the time derivative of the displacement.

We extend the solution (as piecewise constant) to the whole time interval $(0, T]$ using the more convenient notation

$$
\begin{aligned}
& p^{[r]}=\bar{p}^{i} \quad \text { in } \quad\left(t_{i-1}, t_{i}\right], \quad i=1, \ldots, r \\
& \mathbf{u}^{[r]}=\overline{\mathbf{u}}^{i} \text { in }\left(t_{i-1}, t_{i}\right], \quad i=1, \ldots, r .
\end{aligned}
$$

We also utilize this notation for the data, i.e., we construct $\mathbf{F}^{[r]}, \mathbf{g}^{[r]}, S^{[r]}$ and $\psi^{[r]}$ as above on $[0, T]$.

The a priori estimates above yield that the piecewise (in time) constant solution to (36)-(38) on $V \times \mathbf{V}$ enjoys the bounds

$$
\begin{array}{r}
\left\|p^{[r]}\right\|_{L^{2}(0, T ; V)} \leq C \\
\left\|\mathbf{u}^{[r]}\right\|_{L^{2}(0, T ; \mathbf{V})} \leq C \\
\left\|\left(\mathbf{u}^{[r]}\right)_{\Delta t}\right\|_{L^{2}(0, T ; \mathbf{V})} \leq C \\
\sup _{t \in[0, T]}\left\|\mathbf{u}^{[r]}(t)\right\| \mathbf{v} \leq C,
\end{array}
$$


with $\left(\mathbf{u}^{i}\right)_{\Delta t} \equiv[\Delta t]^{-1}\left[\mathbf{u}^{i}-\mathbf{u}^{i-1}\right]$ being the difference quotient with respect to a fixed $\Delta t$. These estimates are uniform in $[r]$ (i.e., as $\Delta t \rightarrow 0$ ) and therefore we can extract weak limit points $p \in L^{2}(0, T ; V), \mathbf{u} \in L^{\infty}((0, T] ; \mathbf{V})$, and $\mathbf{u}^{\sharp} \in L^{2}(0, T ; \mathbf{V})$, with $\mathbf{u}^{\sharp}$ being the weak limit for the sequence $\left(\mathbf{u}^{[r]}\right)_{\Delta t} \in L^{2}(0, T ; \mathbf{V})$.

We note one additional estimate (not following from energy methods) which is the result of Lemma 2:

$$
\left\|\mathbf{u}^{[r]}\right\|_{L^{2}\left(0, T ;\left(H^{1+\varepsilon}(\Omega)\right)^{3}\right)} \leq C
$$

with $C$ being equivalent to those above. Following [55], we provide the following definition which will be used when testing (25) and (26):

Definition 4 Let $f(t) \in C^{\infty}([0, T])$. Define:

$$
\begin{aligned}
f^{i} & \equiv f\left(t_{i}\right), i=1, \ldots, r, \\
f^{r+1} & \equiv f^{r}=f(T), \\
f^{[r]} & \equiv f^{i+1} \quad \text { in }\left(t_{i}, t_{i+1}\right], i=0, \ldots, r-1, \\
\left(f^{[r]}\right)_{\Delta t}^{+} & \equiv \frac{f^{i+2}-f^{i+1}}{\Delta t} \quad \text { in }\left(t_{i}, t_{i+1}\right], i=0, \ldots, r-1,
\end{aligned}
$$

satisfying the following properties:

$$
\left\|f^{[r]}-f\right\|_{L^{2}(0, T)} \leq C \cdot[\Delta t], \quad\left\|\left(f^{[r]}\right)_{\Delta t}^{+}-f^{\prime}\right\|_{L^{2}(0, T)} \leq C \cdot[\Delta t],
$$

where $f^{\prime}$ denotes the derivative of $f$ with respect to its sole scalar argument $t$.

We know that the sequence $\left\{\left(\bar{p}^{i}, \overline{\mathbf{u}}^{i}\right)\right\}$ satisfies the system (39)-(40) for any $(q, \mathbf{w}) \in \mathbb{V}$. Let $q \in W^{1, \infty}(\Omega) \cap V$ and multiply the aforementioned relations by $f^{i}$, simplify, and sum $i=1, . ., r$ to obtain:

$$
\begin{aligned}
\delta \sum_{i=1}^{r} a\left(\left(\overline{\mathbf{u}}^{i}\right)_{\Delta t}, \mathbf{w}\right) f^{i} \cdot[\Delta t] & +\sum_{i=1}^{r} a\left(\overline{\mathbf{u}}^{i}, \mathbf{w}\right) f^{i} \cdot[\Delta t]-\sum_{i=1}^{r}\left(\bar{p}^{i}, \nabla \cdot \mathbf{w}\right) f^{i} \cdot[\Delta t] \\
& =\sum_{i=1}^{r}\left\langle\mathbf{g}^{i}, \mathbf{w}\right\rangle_{\Gamma_{N}} f^{i} \cdot[\Delta t]-\sum_{i=1}^{r}\left(\mathbf{F}^{i}, \mathbf{w}\right) f^{i} \cdot[\Delta t] \\
\sum_{i=1}^{r}\left(k\left(\nabla \cdot \overline{\mathbf{u}}^{i}\right) \nabla \bar{p}^{i}, \nabla q\right) f^{i} \cdot[\Delta t] & +\sum_{i=1}^{r}\left(\nabla \cdot\left(\overline{\mathbf{u}}^{i}\right)_{\Delta t}, q\right) f^{i} \cdot[\Delta t] \\
& =-\sum_{i=1}^{r}\left\langle\psi^{i}, q\right\rangle_{\Gamma_{D, v}} f^{i} \cdot[\Delta t]+\sum_{i=1}^{r}\left(S^{i}, q\right) f^{i} \cdot[\Delta t] .
\end{aligned}
$$


We can identify the sums as integrals in time:

$$
\begin{aligned}
\delta \int_{0}^{T} a\left(\left(\overline{\mathbf{u}}^{[r]}\right)_{\Delta t}, \mathbf{w}\right) f^{[r]} d t & +\int_{0}^{T} a\left(\overline{\mathbf{u}}^{[r]}, \mathbf{w}\right) f^{[r]} d t-\int_{0}^{T}\left(\bar{p}^{[r]}, \nabla \cdot \mathbf{w}\right) f^{[r]} d t \\
& =\int_{0}^{T}\left\langle\mathbf{g}^{[r]}, \mathbf{w}\right\rangle_{\Gamma_{N}} f^{[r]} d t-\int_{0}^{T}\left(\mathbf{F}^{[r]}, \mathbf{w}\right) f^{[r]} d t \\
\int_{0}^{T}\left(k\left(\nabla \cdot \overline{\mathbf{u}}^{[r]}\right) \nabla \bar{p}^{[r]}, \nabla q\right) f^{[r]} d t & +\int_{0}^{T}\left(\nabla \cdot\left(\overline{\mathbf{u}}^{[r]}\right)_{\Delta t}, q\right) f^{[r]} d t \\
& =-\int_{0}^{T}\left\langle\psi^{[r]}, q\right\rangle_{\Gamma_{D, v}} f^{[r]} d t+\int_{0}^{T}\left(S^{[r]}, q\right) f^{[r]} d t .
\end{aligned}
$$

Using the estimates in (58), and adding and subtracting appropriate terms, it is possible to pass to the limit on the linear terms, thereby identifying weak limit points. For those terms not involving the quotient $\left(\mathbf{u}^{[r]}\right)_{\Delta t}$, this proceeds exactly as in [55, pp. 202-204]. More attention is required when passing to the limit on the nonlinear term showing that

$$
\int_{0}^{T}\left(k\left(\nabla \cdot \overline{\mathbf{u}}^{[r]}\right) \nabla \bar{p}^{[r]}, \nabla q\right) f^{[r]} d t \rightarrow \int_{0}^{T}(k(\nabla \cdot \mathbf{u}) \nabla p, \nabla q) f(t) d t .
$$

Remark 14 This is the step in the proof where the nonlinearity most significantly affects the limit passage in the construction of weak solutions. In this step, the elliptic regularity in Lemma 2 is crucial. We require that $B: V \rightarrow H^{\varepsilon}(\Omega)$ in order to gain compactness via the Aubin-Lions Lemma.

To do this, we will consider a particular choice of "antiderivative" of $\left(\mathbf{u}^{[r]}\right)_{\Delta t}$ (following [6, p. 1260]) which will allow us to use the Aubin-Lions Lemma for a stronger convergence of $\nabla \cdot \mathbf{u}^{[r]}$ as $r \rightarrow \infty$. Given the estimates in (53), we have:

Lemma $\mathbf{8}$ For the sequence $\mathbf{u}^{[r]} \in \mathbf{V}$ (as in (53)-(56)) such that $\mathbf{u}^{[r]} \rightarrow \mathbf{u}$ in $L^{2}(0, T ; \mathbf{V})$, we also have that $\nabla \cdot \mathbf{u}^{[r]} \rightarrow \nabla \cdot \mathbf{u}$ in $L^{2}\left(0, T ; L^{2}(\Omega)\right)$.

Proof (of Lemma 8) We introduce the piecewise linear function:

$$
L\left[\nabla \cdot \mathbf{u}^{[r]}\right]=L\left[\nabla \cdot \overline{\mathbf{u}}^{i}\right], \quad \text { on } \quad\left(t_{i-1}, t_{i}\right], i=1, \ldots, r
$$

where

$$
\begin{aligned}
L\left[\nabla \cdot \overline{\mathbf{u}}^{i}\right] & =\left[\frac{\nabla \cdot \overline{\mathbf{u}}^{i}-\nabla \cdot \overline{\mathbf{u}}^{i-1}}{\Delta t}\right]\left(t-t_{i-1}\right)+\nabla \cdot \overline{\mathbf{u}}^{i-1} \\
& =(\nabla \cdot \overline{\mathbf{u}})_{\Delta t}\left(t-t_{i-1}\right)+\nabla \cdot \overline{\mathbf{u}}^{i-1}, \quad \text { on }\left(t_{i-1}, t_{i}\right] .
\end{aligned}
$$

With this notation, we have:

$$
\frac{d}{d t}\left(L\left[\nabla \cdot \mathbf{u}^{[r]}\right]\right)=\left(\nabla \cdot \mathbf{u}^{[r]}\right)_{\Delta t}
$$


Owing to Lemma 7, we immediately obtain the uniform bound in $r$ :

$$
\left\|\frac{d}{d t}\left(L\left[\nabla \cdot \mathbf{u}^{[r]}\right]\right)\right\|_{L^{2}\left(0, T ; L^{2}(\Omega)\right)}=\left\|\left(\nabla \cdot \mathbf{u}^{[r]}\right)_{\Delta t}\right\|_{L^{2}\left(0, T ; L^{2}(\Omega)\right)} \leq C .
$$

Now we note that:

$$
\left\|L\left[\nabla \cdot \overline{\mathbf{u}}^{i}\right]\right\|_{\varepsilon} \leq[\Delta t] \cdot\left\|\left(\nabla \cdot \overline{\mathbf{u}}^{i}\right)_{\Delta t}\right\|_{\varepsilon}+\left\|\nabla \cdot \overline{\mathbf{u}}^{i-1}\right\|_{\varepsilon} .
$$

Moreover, via the continuous mapping $B: L^{2}(\Omega) \rightarrow H^{\varepsilon}(\Omega)$ (see Section 3.3), with $\nabla p^{[r]} \in L^{2}\left(0, T ; L^{2}(\Omega)\right)$, Lemma 7 implies that

$$
\left\|L\left[\nabla \cdot \mathbf{u}^{[r]}\right]\right\|_{L^{2}\left(0, T ; H^{\varepsilon}(\Omega)\right)} \leq C,
$$

where $C$ has the same dependencies as in (53)-(56). Thus, we know that there exist $\mathbf{v} \in L^{2}\left(0, T ; H^{\varepsilon}(\Omega)\right)$ and $\mathbf{v}^{\prime} \in L^{2}\left(0, T ; L^{2}(\Omega)\right)$ such that

$$
L\left[\nabla \cdot \mathbf{u}^{[r]}\right] \rightarrow \mathbf{v}
$$

and

$$
\frac{d}{d t}\left(L\left[\nabla \cdot \mathbf{u}^{[r]}\right]\right) \rightarrow \mathbf{v}^{\prime}
$$

By the Aubin-Lions Lemma, possibly along a subsequence, we have $L[\nabla$. $\left.\mathbf{u}^{[r]}\right] \rightarrow \mathbf{v}$ in the sense of $L^{2}\left(0, T ; L^{2}(\Omega)\right)$. From the piecewise structure of $L\left[\nabla \cdot \mathbf{u}^{[r]}\right]$ we have that

$$
L\left[f^{[r]}(t)\right] \rightarrow f(t) \text { as } r \rightarrow \infty
$$

for any $f(t)$ piecewise continuous. Thus, due to the uniqueness of the limit $\mathbf{v}=\nabla$. $\mathbf{u}$, the weak convergence $\mathbf{u}^{[r]} \rightarrow \mathbf{u} \in \mathbf{V}$ is improved to strong convergence (possibly along a subsequence):

$$
\nabla \cdot \mathbf{u}^{[r]} \rightarrow \nabla \cdot \mathbf{u} \text { in } L^{2}\left(0, T ; L^{2}(\Omega)\right) .
$$

We now consider the difference

$$
\begin{aligned}
& \int_{0}^{T}\left(k\left(\nabla \cdot \overline{\mathbf{u}}^{[r]}\right) \nabla \bar{p}^{[r]}, \nabla q\right) f^{[r]} d t-\int_{0}^{T}(k(\nabla \cdot \mathbf{u}) \nabla p, \nabla q) f(t) d t \\
= & \int_{0}^{T}\left(k\left(\nabla \cdot \mathbf{u}^{[r]}\right) \nabla p^{[r]}, \nabla q\right)\left[f^{[r]}-f\right] d t \\
& +\int_{0}^{T}\left(k\left(\nabla \cdot \mathbf{u}^{[r]}\right) \nabla\left[p^{[r]}-p\right], \nabla q\right) f d t \\
& +\int_{0}^{T}\left(\left[k\left(\nabla \cdot \mathbf{u}^{[r]}\right)-k(\nabla \cdot \mathbf{u})\right] \nabla p, \nabla q\right) f d t .
\end{aligned}
$$

We note that, as $r \rightarrow \infty$, from the properties of $f^{[r]}$ it follows that $\left|\int_{0}^{T}\left(k\left(\nabla \cdot \mathbf{u}^{[r]}\right) \nabla p^{[r]}, \nabla q\right)\left[f^{[r]}-f\right] d t\right| \leq \widehat{\kappa}\left\|p^{[r]}\right\|_{L^{2}(0, T ; V)}\|q\|_{V}\left\|f-f^{[r]}\right\|_{L^{2}(0, T)} \rightarrow 0$, 
from the weak convergence $p^{[r]} \rightarrow p$ in $L^{2}(0, T ; V)$ it follows that

$$
\left.\left|\int_{0}^{T}\left(k\left(\nabla \cdot \mathbf{u}^{[r]}\right) \nabla\left[p^{[r]}-p\right], \nabla q\right) f d t\right| \leq C(\widehat{\kappa}) \mid\left(\left[p^{[r]}-p\right], q f\right)\right)_{L^{2}(0, T ; V)} \mid \rightarrow 0,
$$

and by the Nemytskii property of $k(\cdot)$, since $\nabla \cdot \mathbf{u}^{[r]} \rightarrow \nabla \cdot \mathbf{u}$ strongly in $L^{2}\left(0, T ; L^{2}(\Omega)\right)$, and considering that $q \in V \cap W^{1, \infty}(\Omega), f \in C^{\infty}([0, T])$ it follows that

$$
\begin{aligned}
& \left|\int_{0}^{T}\left(\left[k\left(\nabla \cdot \mathbf{u}^{[r]}\right)-k(\nabla \cdot \mathbf{u})\right] \nabla p, \nabla q\right) f d t\right| \\
& \quad \leq C(q, f)|| k\left(\nabla \cdot \mathbf{u}^{[r]}\right)-k(\nabla \cdot \mathbf{u})\left\|_{L^{2}\left(0, T ; L^{2}(\Omega)\right)}\right\| \nabla p \|_{L^{2}\left(0, T ; L^{2}(\Omega)\right)} \rightarrow 0 .
\end{aligned}
$$

\section{Step 5: Limit point identification}

Thus, we have the following identity for the weak limits (identified above), which holds for all test functions of the form $\mathbf{w} f$ and $q f$ with $w \in \mathbf{V}, q \in V \cap W^{1, \infty}(\Omega)$, and $f \in C^{\infty}([0, T])$ :

$$
\begin{aligned}
\delta \int_{0}^{T} a\left(\mathbf{u}^{\sharp}, \mathbf{w}\right) f d t & +\int_{0}^{T} a(\mathbf{u}, \mathbf{w}) f d t-\int_{0}^{T}(p, \nabla \cdot \mathbf{w}) f d t \\
& =\int_{0}^{T}\langle\mathbf{g}, \mathbf{w}\rangle_{\Gamma_{N}} f d t-\int_{0}^{T}(\mathbf{F}, \mathbf{w}) f d t \\
\int_{0}^{T}(k(\nabla \cdot \mathbf{u}) \nabla p, \nabla q) f d t & +\int_{0}^{T}\left(\nabla \cdot \mathbf{u}^{\sharp}, q\right) f d t \\
& =-\int_{0}^{T}\langle\psi, q\rangle_{\Gamma_{D, v}} f d t+\int_{0}^{T}(S, q) f d t .
\end{aligned}
$$

We must now identify the weak limit for the difference quotient (in time) $\mathbf{u}^{\sharp}$ with the distributional derivative in time of $\mathbf{u}$. Now, consider the test function $\mathbf{w} f^{i}$, as above. Then

$$
\sum_{i=1}^{r} a\left(\overline{\mathbf{u}}^{i}-\overline{\mathbf{u}}^{i-1}, \mathbf{w}\right) f^{i}=a\left(\overline{\mathbf{u}}^{r}, \mathbf{w}\right) f^{r}-a\left(\mathbf{u}^{0}, \mathbf{w}\right) f^{1}-\sum_{i=1}^{r-1} a\left(\overline{\mathbf{u}}^{i}, \mathbf{w}\right)\left(f^{i+1}-f^{i}\right) .
$$

Due to the fact that $\left(f^{[r]}\right)_{\Delta t}^{+}=0$ on $\left(t_{r-1}, t_{r}\right]$, we have that

$$
\sum_{i=1}^{r-1} a\left(\overline{\mathbf{u}}^{i}, \mathbf{w}\right)\left(f^{i+1}-f^{i}\right)=\Delta t \sum_{i=1}^{r-1} a\left(\overline{\mathbf{u}}^{i}, \mathbf{w}\right) \frac{f^{i+1}-f^{i}}{\Delta t}=\int_{0}^{T} a\left(\mathbf{u}^{[r]}, \mathbf{w}\right)\left[\left(f^{[r]}\right)_{\Delta t}^{+}\right] d t .
$$

Again, using the linear nature of this term and the boundedness of the sequences in (53), we see that as $r \rightarrow \infty$ :

$$
\begin{aligned}
\delta \sum_{i=1}^{r} a\left(\left(\overline{\mathbf{u}}^{i}\right)_{\Delta t}, \mathbf{w}\right) f^{i} \cdot[\Delta t] & \rightarrow \\
& \delta a(\mathbf{u}(T), \mathbf{w}) f(T)-\delta a\left(\mathbf{u}^{0}, \mathbf{w}\right) f(0)-\delta \int_{0}^{T} a(\mathbf{u}, \mathbf{w}) f^{\prime} d t .
\end{aligned}
$$


But, since this holds for all $f \in C_{0}^{\infty}(0, T)$, this implies that

$$
\delta \sum_{i=1}^{r} a\left(\left(\overline{\mathbf{u}}^{i}\right)_{\Delta t}, \mathbf{w}\right) f^{i} \cdot[\Delta t] \rightarrow \delta \int_{0}^{T} a\left(\mathbf{u}_{t}, \mathbf{w}\right) f d t,
$$

where $\mathbf{u}_{t}$ is the distributional derivative in time. From this we can infer that:

$$
\int_{0}^{T} a\left(\mathbf{u}_{t}, \mathbf{w}\right) f d t=\int_{0}^{T} a\left(\mathbf{u}^{\sharp}, \mathbf{w}\right) f d t .
$$

\section{Step 6: Properties of the solution}

Since functions of the form $\mathbf{w} f$ with $w \in \mathbf{V}$ and $f \in C^{\infty}([0, T])$ are dense in $L^{2}([0, T] ; \mathbf{V})$, it follows that $\mathbf{u}_{t}=\mathbf{u}^{\sharp}$ in the sense of $L^{2}\left(0, T ;\left(L^{2}(\Omega)\right)^{3}\right)$. Moreover, since $\mathbf{u}^{\sharp}$ is the weak limit of the sequence $\left(\mathbf{u}^{[r]}\right)_{\Delta t} \in L^{2}(0, T ; \mathbf{V})$, we have that $\mathbf{u}^{\sharp} \in L^{2}(0, T ; \mathbf{V})$, which, by uniqueness of limits, implies that $\mathbf{u}_{t} \in L^{2}(0, T ; \mathbf{V})$ as well.

Remark 15 This identification and additional regularity for $\mathbf{u}_{t} \in L^{2}(0, T ; \mathbf{V})$ is possible because $\delta$ is strictly positive and, indeed, it cannot be attained in the case $\delta=0$ considered below. The original work in [55], as well as the model considered in [6], only deal with the case $\delta=0$, and consequently identify $\nabla \cdot \mathbf{u}_{t}$ in the weaker space $L^{2}\left(0, T ; V^{\prime}\right)$, as in Step 5 of Section 3.4 .2 below.

Remark 16 The test function $\left(f^{[r]}\right)_{\Delta t}^{+}$is used in both viscoelastic and purely elastic cases, but for different reasons. When $\delta>0$, it is needed to identify the weak limit of the sequence in (50). In contrast, when $\delta=0$, it is used to perform the summation by parts in the time-discretized pressure equation (40).

Thus, we have constructed a solution $\mathbf{u} \in L^{2}(0, T ; \mathbf{V}), \mathbf{u}_{t} \in L^{2}(0, T ; \mathbf{V})$ and $p \in$ $L^{2}(0, T ; V)$ which satisfies (25)-(26). Additionally, we note that $\mathbf{u} \in H^{1}(0, T ; \mathbf{V})$ and so $\nabla \cdot \mathbf{u} \in H^{1}\left(0, T ; L^{2}(\Omega)\right)$. Thus, by [18], it follows that $\mathbf{u} \in C([0, T] ; \mathbf{V})$ and $\nabla \cdot \mathbf{u} \in C\left([0, T] ; L^{2}(\Omega)\right)$. We note that the property $\mathbf{u} \in L^{\infty}([0, T] ; \mathbf{V})$ actually follows from the a priori bound in (56) in the limit; additionally, this can be seen in Section 3.5 utilizing specific test functions: as $\mathbf{u}_{t} \in L^{2}(0, T ; \mathbf{V})$, and test functions of the form $\mathbf{w} f(\cdot)$ (as above) are dense in this space, we may consider both $\mathbf{u}$ and $\mathbf{u}_{t}$ as valid test functions in (25)-(26). This provides energy estimates, as well as energy identities, for solutions (the calculations and formal statements have been detailed in Section 3.5).

Remark 17 Obtaining a priori estimates is more subtle in the $\delta=0$ case as we cannot utilize $\mathbf{u}_{t}$ as a test function in (27)-(28), since functions in $L^{2}\left(0, T ;\left(L^{2}(\Omega)\right)^{3}\right)$ are not valid "multipliers" for the elasticity/momentum equation.

\section{Step 7: Recovery of initial condition}

To recover the initial condition from the constructed solution we start from the momentum equation (25). For any $\mathbf{w} \in \mathbf{V}$, we can define

$$
\begin{aligned}
G(t) & \equiv \delta a(\mathbf{u}(t), \mathbf{w}) \\
H(t) & \equiv-\left(a(\mathbf{u}(t), \mathbf{w})+(p(t), \nabla \cdot \mathbf{w})+\langle\mathbf{g}(t), \mathbf{w}\rangle_{\Gamma_{N}}-(\mathbf{F}(t), \mathbf{w})\right. \\
F(t) & \equiv \int_{0}^{t} H(\tau) d \tau
\end{aligned}
$$


and note that $F(t)$ is absolutely continuous on $[0, T]$ with $F^{\prime}(t)=H(t)$ a.e. $(0, T)$. Utilizing these definitions in (25), we obtain

$$
\int_{0}^{T}\left(G^{\prime}(t)-F^{\prime}(t)\right) f(t) d t=0 \quad \forall f \in C_{0}^{\infty}(0, T),
$$

and this implies that $G$ and $F$ differ by a constant, i.e. $G-F=c$. By considering $f \in C^{\infty}([0, T])$ with $f(0)=1$ and $f(T)=0$, recalling (72)-(73), and integrating by parts in time in (25), we obtain

$$
\begin{array}{rl}
-\delta \int_{0}^{T} & a(\mathbf{u}, \mathbf{w}) f^{\prime} d t-\delta a\left(\mathbf{u}^{0}, \mathbf{w}\right)= \\
& \quad-\int_{0}^{T} a(\mathbf{u}, \mathbf{w}) f d t+\int_{0}^{T}(p, \nabla \cdot \mathbf{w}) f d t+\int_{0}^{T}\langle\mathbf{g}, \mathbf{w}\rangle_{\Gamma_{N}} d t-\int_{0}^{T}(\mathbf{F}, \mathbf{w}) f d t
\end{array}
$$

for all $\mathbf{w} \in \mathbf{V}$. This can be rewritten as

$$
-\int_{0}^{T} G f^{\prime} d t+\int_{0}^{T} H f d t=\delta a\left(\mathbf{u}^{0}, \mathbf{w}\right) .
$$

Integrating by parts in time and using (77) we have:

$$
\int_{0}^{T} H f d t+\int_{0}^{T} F^{\prime} f d t-\left.[(F(t)+c) f(t)]\right|_{0} ^{T}=\delta a\left(\mathbf{u}^{0}, \mathbf{w}\right) .
$$

By identifying $F^{\prime}=H$ a.e. $t$, choosing $T=0$ and recalling that $f(0)=1$, from (78) it follows that $c=\delta a\left(\mathbf{u}^{0}, \mathbf{w}\right)$ and, consequently

$$
\begin{aligned}
\delta a(\mathbf{u}(t), \mathbf{w})- & \delta a\left(\mathbf{u}^{0}, \mathbf{w}\right)= \\
& \int_{0}^{T}\left[-\left(a(\mathbf{u}(t), \mathbf{w})+(p(t), \nabla \cdot \mathbf{w})+\langle\mathbf{g}(t), \mathbf{w}\rangle_{\Gamma_{N}}-(\mathbf{F}(t), \mathbf{w})\right] d t\right.
\end{aligned}
$$

for all $\mathbf{w} \in \mathbf{V}$ and a.e. $(0, T)$. Choosing $T=0$, we see that

$$
a(\mathbf{u}(0), \mathbf{w})=a\left(\mathbf{u}^{0}, \mathbf{w}\right), \mathbf{w} \in \mathbf{V},
$$

which yields that $\mathbf{u}(0)=\mathbf{u}^{0}$ in the sense of $\mathbf{V}$. Additionally, this yields that $\nabla$. $\mathbf{u}(0)=\nabla \cdot \mathbf{u}^{0}$, and, since $\nabla \cdot \mathbf{u}^{0}=d_{0}$ by the compatibility of initial conditions, we have satisfied both initial conditions.

This completes the proof of Theorem 1.

3.4.2 The elastic case: $\delta=0$

Theorem 2 [Existence of E-Solutions] Consider (15)-(22) with $\delta=0$. Let Assumption 3.1 hold, and consider data of the form:

$$
\begin{array}{cl}
\mathbf{F} \in H^{1}\left(0, T ;\left(L^{2}(\Omega)\right)^{3}\right), & S \in L^{2}\left(0, T ; L^{2}(\Omega)\right), \\
\mathbf{g} \in H^{1}\left(0, T ;\left(H^{1 / 2}\left(\Gamma_{N}\right)\right)^{3}\right), & \psi \in L^{2}\left(0, T ; L^{2}\left(\Gamma_{D, v}\right)\right) .
\end{array}
$$

Then there exists an E-solution (in the sense of (27)-(28)) satisfying

$$
\sup _{t \in[0, T]} E(\mathbf{u}(t))+\int_{0}^{T}[E(p(t))+E(\mathbf{u}(t))] d t \leq C_{1}\left[E(\mathbf{u}(0))+\left.D A T A_{0}\right|_{0} ^{T}\right] e^{C_{2} T} .
$$




\section{Step 1: The discretized problem}

We utilize the same partition of $[0, T]$ into $r$ sub-intervals, yielding $\Delta t=T / r$ and $t_{i}=i \Delta t, i=0,1, \ldots, r$. As in the case $\delta>0$, we define

$$
\psi^{i} \equiv[\Delta t]^{-1} \int_{t_{i-1}}^{t_{i}} \psi(x, t) d t
$$

with $S^{i}$ defined analogously. However, due to their higher time-regularity, we define

$$
\widehat{\mathbf{g}}^{i} \equiv \mathbf{g}\left(x, t_{i}\right),
$$

with $\widehat{\mathbf{F}}^{i}$ defined analogously. We now define a weak form of the temporal semidiscretized problem when $\delta=0$ as:

$$
\begin{aligned}
a\left(\mathbf{u}^{i}, \mathbf{w}\right)-\left(p^{i}, \nabla \cdot \mathbf{w}\right) & =\left\langle\widehat{\mathbf{g}}^{i}, \mathbf{w}\right\rangle_{\Gamma_{N}}-\left(\widehat{\mathbf{F}}^{i}, \mathbf{w}\right) \\
{[\Delta t]\left(k\left(\nabla \cdot \mathbf{u}^{i}\right) \nabla p^{i}, \nabla q\right) } & +\left(\nabla \cdot \mathbf{u}^{i}, q\right) \\
& =\left(\nabla \cdot \mathbf{u}^{i-1}, q\right)-[\Delta t]\left\langle\psi^{i}, q\right\rangle_{\Gamma_{D, v}}+[\Delta t]\left(S^{i}, q\right) \\
\nabla \cdot \mathbf{u}(0) & =d_{0}
\end{aligned}
$$

for all $(\mathbf{w}, q) \in \mathbf{V} \times V$.

Remark 18 Depending on whether $\delta>0$ or $\delta=0$, the resulting natural choice for the time scaling of the temporal semi-discretized weak problem noticeably differs. This is clear when comparing (36)-(38) with (81)-(83).

\section{Step 2: Solving the fully discretized problem}

The solution of the discretized problem in the case $\delta=0$ mirrors that of $\delta>0$. In the $\delta=0$ case, we again take the projection of $\mathbf{u}^{0}$ onto $\mathbf{V}_{h}$, resulting in $\mathbf{u}_{h}^{0}$; since $\nabla \cdot \mathbf{u}^{0}=d_{0}$, we set $d_{0, h}=\nabla \cdot \mathbf{u}_{h}^{0}$ (see Remark 3). In (81)-(83), we observe that $\left(\mathbf{u}_{h}^{1}, p_{h}^{1}\right)$ are obtained from the data $\nabla \cdot \mathbf{u}_{h}^{0}=d_{0, h}, \psi^{0}, S^{0}, \widehat{\mathbf{g}}^{0}$, and $\widehat{\mathbf{F}}^{0}$.

We similarly define a map $G_{0}: \mathbb{V}_{h} \rightarrow \mathbb{V}_{h}$ by the bilinear form below: for $\left(p^{i}, \mathbf{u}^{i}\right) \in \mathbb{V}_{h}$

$$
\begin{aligned}
\left(G_{0}\left[\begin{array}{c}
p^{i} \\
\mathbf{u}^{i}
\end{array}\right],\left[\begin{array}{c}
q \\
\mathbf{w}
\end{array}\right]\right)= & a\left(\mathbf{u}^{i}, \mathbf{w}\right)-\left(p^{i}, \nabla \cdot \mathbf{w}\right)+[\Delta t]\left(k\left(\nabla \cdot \mathbf{u}^{i}\right) \nabla p^{i}, \nabla q\right) \\
& +\left(\nabla \cdot \mathbf{u}^{i}, q\right)-\left(\nabla \cdot \mathbf{u}^{i-1}, q\right) \\
& +[\Delta t]\left\langle\psi^{i}, q\right\rangle_{\Gamma_{D, v}}-[\Delta t]\left(S^{i}, q\right)-\left\langle\widehat{\mathbf{g}}^{i}, \mathbf{w}\right\rangle_{\Gamma_{N}}-\left(\widehat{\mathbf{F}}^{i}, \mathbf{w}\right)
\end{aligned}
$$

for all $(q, \mathbf{w}) \in \mathbb{V}_{h}$. The analysis of $G_{0}$ in relation to the corresponding problem on $\mathbb{V}_{h}$ (and associated estimates) proceeds precisely as in Step 2 for the $\delta>0$ case. Thus there exists a point $\left(\bar{p}_{h}^{i}, \overline{\mathbf{u}}_{h}^{i}\right) \in \mathbb{V}_{h}$ satisfying:

$$
\left(G_{0}\left[\begin{array}{c}
\bar{p}_{h}^{i} \\
\overline{\mathbf{u}}_{h}^{i}
\end{array}\right],\left[\begin{array}{c}
q \\
\mathbf{w}
\end{array}\right]\right)=0
$$


for all $(q, \mathbf{w}) \in \mathbb{V}$. Moreover, $\left(\bar{p}_{h}^{i}, \overline{\mathbf{u}}_{h}^{i}\right)$ has the property that:

$$
\left\|\left[\begin{array}{c}
\bar{p}_{h}^{i} \\
\overline{\mathbf{u}}_{h}^{i}
\end{array}\right]\right\|_{\mathbb{V}_{h}}^{2} \leq \frac{c\left[\left\|\widehat{\mathbf{g}}^{i}\right\|_{L^{2}\left(\Gamma_{N}\right)}^{2}+\left\|\widehat{\mathbf{F}}^{i}\right\|_{0}^{2}+\left\|\mathbf{u}^{i-1}\right\|_{1}^{2}+[\Delta t]\left(\left\|\psi^{i}\right\|_{L^{2}\left(\Gamma_{D, v}\right)}^{2}+\left\|S^{i}\right\|_{0}^{2}\right)\right]}{C(\kappa)[\Delta t]} .
$$

We have also, then, produced a weak solution of the approximate problem (81)(83) on $\mathbb{V}_{h}$ (for each $i, i=1, \ldots, r$ ) from the data given for $i-1$, and this solution enjoys a uniform bound in $\mathbb{V}_{h} \subset V \times \mathbf{V}$ with respect to $h$ via (85).

\section{Step 3: Limit passage in space}

Since the additional time regularity due to the viscoelastic term does not influence the passage to the limit in space, we can proceed analogously to what described in Step 3 for the $\delta>0$ case, thus obtaining a solution to (81)-(83) on $\mathbb{V}$ as stated in the following Lemma.

Lemma 9 Consider data of the form $\left(\mathbf{u}^{i-1}, p^{i-1}, \psi^{i}, \widehat{\mathbf{g}}^{i}, \widehat{\mathbf{F}}^{i}, S^{i}\right)$-with $\left(p^{i-1}, \mathbf{u}^{i-1}\right) \in$ $\mathbb{V}$. Then there is a solution $\left(\bar{p}^{i}, \overline{\mathbf{u}}^{i}\right) \in V \times \mathbf{V}$ that satisfies (81)-(83) for all test functions $(\mathbf{w}, q) \in \mathbb{V}$.

\section{Step 4: Limit passage in time}

The passage to the limit in time is more subtle in the $\delta=0$ case, owing to the natural lack of smoothness in time for solutions. Analogously to Lemma 7, the key step is obtaining the following set of upper bounds that are uniform in $r$.

Lemma 10 For each $i=1, \ldots, r$ solutions to (81)-(83) on $V \times \mathbf{V}$ enjoy the estimates

$$
\begin{array}{r}
{[\Delta t] \sum_{i=1}^{r}\left\|\bar{p}^{i}\right\|_{1}^{2} \leq C} \\
\left\|\overline{\mathbf{u}}^{i}\right\|_{1}^{2} \leq C \\
{[\Delta t] \sum_{i=1}^{r}\left\|\overline{\mathbf{u}}^{i}\right\|_{1}^{2} \leq C}
\end{array}
$$

where the constant $C$ above depends on $T, E\left(\mathbf{u}^{0}\right)$, and DATA $\left.0\right|_{0} ^{T}$ (as in (124)).

Proof (of Lemma 10) The following identities will be useful for the analysis:

$$
\begin{gathered}
a\left(\mathbf{w}^{i}, \mathbf{w}^{i}-\mathbf{w}^{i-1}\right)=\frac{1}{2} a\left(\mathbf{w}^{i}, \mathbf{w}^{i}\right)-\frac{1}{2} a\left(\mathbf{w}^{i-1}, \mathbf{w}^{i-1}\right)+\frac{1}{2} a\left(\mathbf{w}^{i}-\mathbf{w}^{i-1}, \mathbf{w}^{i}-\mathbf{w}^{i-1}\right) \\
\sum_{i=1}^{j}\left(\mathbf{G}^{i}, \mathbf{w}^{i}-\mathbf{w}^{i-1}\right)=\left(\mathbf{G}^{j}, \mathbf{w}^{j}\right)-\left(\mathbf{G}^{1}, \mathbf{w}^{0}\right)-\sum_{i=1}^{j-1}\left(\mathbf{G}^{i+1}-\mathbf{G}^{i}, \mathbf{w}^{i}\right)
\end{gathered}
$$

where $\mathbf{G}$ and $\mathbf{w}$ are arbitrary functions.

For each $i=1, \ldots, r$, let us test (81) for the solution $\left(\bar{p}^{i}, \overline{\mathbf{u}}^{i}\right)$ with $\mathbf{w}=\overline{\mathbf{u}}^{i}-\overline{\mathbf{u}}^{i-1}$ :

$$
a\left(\overline{\mathbf{u}}^{i},\left[\overline{\mathbf{u}}^{i}-\overline{\mathbf{u}}^{i-1}\right]\right)-\left(\bar{p}^{i}, \nabla \cdot\left[\overline{\mathbf{u}}^{i}-\overline{\mathbf{u}}^{i-1}\right]\right)=\left\langle\widehat{\mathbf{g}}^{i},\left[\overline{\mathbf{u}}^{i}-\overline{\mathbf{u}}^{i-1}\right]\right\rangle_{\Gamma_{N}}-\left(\widehat{\mathbf{F}}^{i},\left[\overline{\mathbf{u}}^{i}-\overline{\mathbf{u}}^{i-1}\right]\right) .
$$


Using (89) and simplifying we have:

$$
\begin{aligned}
& \frac{1}{2} a\left(\overline{\mathbf{u}}^{i}, \overline{\mathbf{u}}^{i}\right)+\frac{1}{2} a\left(\overline{\mathbf{u}}^{i}-\overline{\mathbf{u}}^{i-1}, \overline{\mathbf{u}}^{i}-\overline{\mathbf{u}}^{i-1}\right)-\left(\bar{p}^{i}, \nabla \cdot \overline{\mathbf{u}}^{i}\right) \\
& \quad=-\left(\bar{p}^{i}, \nabla \cdot \overline{\mathbf{u}}^{i-1}\right)+\frac{1}{2} a\left(\overline{\mathbf{u}}^{i-1}, \overline{\mathbf{u}}^{i-1}\right)+\left\langle\widehat{\mathbf{g}}^{i},\left[\overline{\mathbf{u}}^{i}-\overline{\mathbf{u}}^{i-1}\right]\right\rangle_{\Gamma_{N}}-\left(\widehat{\mathbf{F}}^{i},\left[\overline{\mathbf{u}}^{i}-\overline{\mathbf{u}}^{i-1}\right]\right) .
\end{aligned}
$$

Testing (83) with $q=\bar{p}^{i}$, we have:

$$
\begin{aligned}
{[\Delta t]\left(k\left(\nabla \cdot \overline{\mathbf{u}}^{i}\right) \nabla \bar{p}^{i}, \nabla \bar{p}^{i}\right) } & +\left(\nabla \cdot \overline{\mathbf{u}}^{i}, \bar{p}^{i}\right) \\
& =\left(\nabla \cdot \overline{\mathbf{u}}^{i-1}, \bar{p}^{i}\right)-[\Delta t]\left\langle\psi^{i}, \bar{p}^{i}\right\rangle_{\Gamma_{D, v}}+[\Delta t]\left(S^{i}, \bar{p}^{i}\right) .
\end{aligned}
$$

Adding (91) and (92), we have the identity:

$$
\begin{aligned}
& \frac{1}{2} a\left(\overline{\mathbf{u}}^{i}, \overline{\mathbf{u}}^{i}\right)+\frac{1}{2} a\left(\overline{\mathbf{u}}^{i}-\overline{\mathbf{u}}^{i-1}, \overline{\mathbf{u}}^{i}-\overline{\mathbf{u}}^{i-1}\right)+[\Delta t]\left(k\left(\nabla \cdot \overline{\mathbf{u}}^{i}\right) \nabla \bar{p}^{i}, \nabla \bar{p}^{i}\right) \\
& =\frac{1}{2} a\left(\overline{\mathbf{u}}^{i-1}, \overline{\mathbf{u}}^{i-1}\right)-[\Delta t]\left\langle\psi^{i}, \bar{p}^{i}\right\rangle_{\Gamma_{D, v}}+[\Delta t]\left(S^{i}, \bar{p}^{i}\right) \\
& \quad+\left\langle\widehat{\mathbf{g}}^{i},\left[\overline{\mathbf{u}}^{i}-\overline{\mathbf{u}}^{i-1}\right]\right\rangle_{\Gamma_{N}}-\left(\widehat{\mathbf{F}}^{i},\left[\overline{\mathbf{u}}^{i}-\overline{\mathbf{u}}^{i-1}\right]\right) .
\end{aligned}
$$

From this key identity, we perform a summation on the index $i$, with $i=1, \ldots, j$, and utilize (90). This results in:

$$
\begin{aligned}
\sum_{i=1}^{j}\left\{E\left(\overline{\mathbf{u}}^{i}\right)\right. & \left.+E\left(\overline{\mathbf{u}}^{i}-\overline{\mathbf{u}}^{i-1}\right)\right\}+\sum_{i=1}^{j} E\left(\bar{p}^{i}\right)[\Delta t] \\
= & \sum_{i=1}^{j} E\left(\overline{\mathbf{u}}^{i-1}\right)+\sum_{i=1}^{j}\left\{\left(S^{i}, \bar{p}^{i}\right)-\left\langle\psi^{i}, \bar{p}^{i}\right\rangle_{\Gamma_{D, v}}\right\}[\Delta t] \\
& -\left(\widehat{\mathbf{F}}^{j}, \overline{\mathbf{u}}^{j}\right)+\left(\widehat{\mathbf{F}}^{1}, \overline{\mathbf{u}}^{0}\right)-\sum_{i=1}^{j-1}\left(\left[\widehat{\mathbf{F}}^{i+1}-\widehat{\mathbf{F}}^{i}\right], \overline{\mathbf{u}}^{i}\right) \\
& +\left\langle\widehat{\mathbf{g}}^{j}, \overline{\mathbf{u}}^{j}\right\rangle_{\Gamma_{N}}-\left\langle\widehat{\mathbf{g}}^{1}, \overline{\mathbf{u}}^{0}\right\rangle_{\Gamma_{N}}+\sum_{i=1}^{j-1}\left\langle\left\langle\widehat{\mathbf{g}}^{i+1}-\widehat{\mathbf{g}}^{i}\right], \overline{\mathbf{u}}^{i}\right\rangle_{\Gamma_{N}} .
\end{aligned}
$$

We will now utilize the structure (and regularity assumptions) of $\widehat{\mathbf{g}}^{i}, \widehat{\mathbf{F}}^{i}, \psi^{i}$, and $S^{i}$ to obtain a priori bounds, uniform in $[r]$. Using (i) Cauchy-Schwarz in space, (ii) the trace theorem, (iii) Bochner's Theorem and Cauchy-Schwarz in time, (iv) Young's inequality as $|a b| \leq \varepsilon a^{2}+\frac{C}{\varepsilon} b^{2}$, and (v) the lower bound on $k(\cdot)$ (see Assumption 3.1), we obtain:

$$
\begin{aligned}
\left|\left\langle\psi^{i}, \bar{p}^{i}\right\rangle[\Delta t]\right| & =\left|[\Delta t]^{-1}\left\langle\int_{t_{i-1}}^{t_{i}} \psi(t) d t, \bar{p}^{i}\right\rangle_{\Gamma_{D, v}}[\Delta t]\right| \\
& \leq C\left\|\int_{t_{i-1}}^{t_{i}} \psi(t) d t||_{0, \Gamma_{D, v}}\right\| \bar{p}^{i} \|_{1} \\
& \leq C[\Delta t]^{1 / 2}\|\psi\|\left\|_{L^{2}\left(t_{i-1}, t_{i} ; L^{2}\left(\Gamma_{D, v}\right)\right)}\right\| \bar{p}^{i} \|_{1} \\
& \leq C_{\varepsilon}\|\psi\|_{L^{2}\left(t_{i-1}, t_{i} ; L^{2}\left(\Gamma_{D, v}\right)\right)}^{2}+\varepsilon E\left(\bar{p}^{i}\right)[\Delta t] .
\end{aligned}
$$


The term $\left(S^{i}, \bar{p}^{i}\right)_{\Omega}$ is handled similarly (using Poincare's inequality, rather than the trace theorem). By the regularity of $\mathbf{g}$, it follows that:

$$
\widehat{\mathbf{g}}^{i+1}-\widehat{\mathbf{g}}^{i}=\int_{t_{i-1}}^{t_{i}} \mathbf{g}_{t}(x, t) d t \text { a.e. on } \Gamma_{N} .
$$

Using Korn's inequality, following analogous steps as above we obtain:

$$
\begin{aligned}
\left|\left\langle\left\langle\widehat{\mathbf{g}}^{i+1}-\widehat{\mathbf{g}}^{i}\right], \overline{\mathbf{u}}^{i}\right\rangle_{\Gamma_{N}}\right| & =\left|\left\langle\int_{t_{i}}^{t_{i+1}} \mathbf{g}^{\prime}(t) d t, \overline{\mathbf{u}}^{i}\right\rangle_{\Gamma_{N}}\right| \\
& \leq C\left\|\int_{t_{i}}^{t_{i+1}} \mathbf{g}^{\prime}(t) d t\right\|_{0, \Gamma_{N}}\left\|\overline{\mathbf{u}}^{i}\right\|_{1} \\
& \leq C[\Delta t]^{1 / 2}\left\|\mathbf{g}^{\prime}\right\|_{L^{2}\left(t_{i}, t_{i+1} ;\left(L^{2}\left(\Gamma_{N}\right)\right)^{3}\right)}\left\|\overline{\mathbf{u}}^{i}\right\|_{1} \\
& \leq C\left\{\left\|\mathbf{g}^{\prime}\right\|_{L^{2}\left(t_{i}, t_{i+1} ;\left(L^{2}\left(\Gamma_{N}\right)\right)^{3}\right)}^{2}+E\left(\overline{\mathbf{u}}^{i}\right)[\Delta t]\right\} .
\end{aligned}
$$

The term $\left(\widehat{\mathbf{F}}^{i+1}-\widehat{\mathbf{F}}^{i}, \overline{\mathbf{u}}^{i}\right)$ is handled similarly. Summing the previous results, and simplifying (96) we have:

$$
\begin{aligned}
\sum_{i=1}^{j} E\left(\overline{\mathbf{u}}^{i}\right)+\sum_{i=1}^{j} E\left(\bar{p}^{i}\right)[\Delta t] \leq & \mathscr{C}\left\{E\left(\mathbf{u}^{0}\right)+\sum_{i=1}^{j-1} E\left(\overline{\mathbf{u}}^{i}\right)+\sum_{i=1}^{j-1} E\left(\overline{\mathbf{u}}^{i}\right)[\Delta t]+\varepsilon \sum_{i=1}^{j-1} E\left(\bar{p}^{i}\right)\right. \\
& +\left\|\mathbf{g}^{\prime}\right\|_{L^{2}\left(0, T ;\left(L^{2}\left(\Gamma_{N}\right)\right)^{3}\right)}^{2}+\left\|\mathbf{F}^{\prime}\right\|_{L^{2}\left(0, T ;\left(L^{2}(\Omega)\right)^{3}\right)} \\
& +\|\mathbf{g}\|_{C\left([0, T] ;\left(L^{2}\left(\Gamma_{N}\right)\right)^{3}\right)}^{2}+\|\mathbf{F}\|_{C\left([0, T] ;\left(L^{2}(\Omega)\right)^{3}\right)}^{2} \\
& \left.+\|\psi\|_{L^{2}\left(0, T ; L^{2}\left(\Gamma_{D, v}\right)\right)}^{2}+\|S\|_{L^{2}\left(0, T ; L^{2}(\Omega)\right)}^{2}\right\} .
\end{aligned}
$$

Simplifying, using the embedding $H^{1}\left(0, T ;\left(L^{2}(D)\right)^{3}\right) \hookrightarrow C\left([0, T] ;\left(L^{2}(D)\right)^{3}\right)$, and possibly scaling $\varepsilon$ (at the cost of up-scaling $\mathscr{C}$ ), we then have:

$$
E\left(\overline{\mathbf{u}}^{j}\right)+\sum_{i=1}^{j} E\left(\bar{p}^{i}\right)[\Delta t] \leq \mathscr{C}_{1}+\mathscr{C}_{2} \sum_{i=1}^{j-1} E\left(\overline{\mathbf{u}}^{i}\right)[\Delta t]
$$

where $\mathscr{C}_{1}$ is a scalar multiple of

$$
\begin{gathered}
E\left(\mathbf{u}^{0}\right)+\|\mathbf{g}\|_{H^{1}\left(0, T ;\left(L^{2}\left(\Gamma_{N}\right)\right)^{3}\right)}^{2}+\|\mathbf{F}\|_{H^{1}\left(0, T ;\left(L^{2}(\Omega)\right)^{3}\right)}^{2}+ \\
\|\psi\|_{L^{2}\left(0, T ; L^{2}\left(\Gamma_{D, v}\right)\right)}^{2}+\|S\|_{L^{2}\left(0, T ; L^{2}(\Omega)\right)}^{2}
\end{gathered}
$$

and $\mathscr{C}_{2}$ is a constant which does not depend on $\mathbf{u}^{0}$ or $[\Delta t]$.

Remark 19 The $\mathscr{C}_{i}$ depend on: the Poincare constant for $\Omega$, the Korn constant, the trace constant, and the lower bound on the permeability $\kappa$. (See Assumption 3.1 and Section 3.6.) 
Finally, we employ the discrete version of Gronwall's Lemma on (99) to obtain:

$$
E\left(\overline{\mathbf{u}}^{j}\right) \leq \mathscr{C}_{1} e^{\mathscr{C}_{2} j}
$$

from which the final conclusion of Lemma 10 follows.

Extending the solution to the whole time interval $(0, T]$ in a piecewise fashion, as before, we have:

$$
\begin{array}{ll}
p^{[r]}=\bar{p}^{i} \quad \text { in } \quad\left(t_{i-1}, t_{i}\right], \quad i=1, \ldots, r \\
\mathbf{u}^{[r]}=\overline{\mathbf{u}}^{i} \text { in }\left(t_{i-1}, t_{i}\right], \quad i=1, \ldots, r .
\end{array}
$$

The a priori estimates above yield that the spatially and temporally discretized solution to (81)-(83) on $V \times \mathbf{V}$ enjoys the bounds

$$
\begin{array}{r}
\left\|p^{[r]}\right\|_{L^{2}(0, T ; V)} \leq C \\
\left\|\mathbf{u}^{[r]}\right\|_{L^{2}(0, T ; \mathbf{V})} \leq C \\
\sup _{t \in[0, T]}\left\|\mathbf{u}^{[r]}(t)\right\| \mathbf{v} \leq C,
\end{array}
$$

which are uniform as $r \rightarrow \infty(\Delta t \rightarrow 0)$. Again, from the elliptic regularity associated with the $B$ mapping, we also have the estimate

$$
\left\|\mathbf{u}^{[r]}\right\|_{L^{2}\left(0, T ;\left(H^{1+\varepsilon}(\Omega)\right)^{3}\right)} \leq C
$$

where the $C$ here is as above. From the bounds in (102)-(104) we identify weak limit points $\mathbf{u} \in L^{2}(0, T ; \mathbf{V})$ and $p \in L^{2}(0, T ; V)$. In (81)-(83), we now consider test functions $\mathbf{w} f$ and $q f$ with $\mathbf{w} \in \mathbf{V}, q \in V$, and $f \in C^{\infty}([0, T])$. We multiply by the appropriate test function and sum each relation from $i=1$ to $i=r$, utilizing the notation introduced in Definition 4.

Note that:

$\sum_{i=1}^{r}\left(\nabla \cdot \overline{\mathbf{u}}^{i}-\nabla \cdot \overline{\mathbf{u}}^{i-1}, q\right) f^{i}=\left(\nabla \cdot \overline{\mathbf{u}}^{r}, q\right) f^{r}-\left(\nabla \cdot \mathbf{u}^{0}, q\right) f^{1}-\sum_{i=1}^{r-1}\left(\nabla \cdot \overline{\mathbf{u}}^{i}, q\right)\left(f^{i+1}-f^{i}\right)$.

Now, due to the fact that $\left(f^{[r]}\right)_{\Delta t}^{+}=0$ on $\left(t_{r-1}, t_{r}\right]$, we have that

$\sum_{i=1}^{r-1}\left(\nabla \cdot \overline{\mathbf{u}}^{i}, q\right)\left(f^{i+1}-f^{i}\right)=\Delta t \sum_{i=1}^{r-1}\left(\nabla \cdot \overline{\mathbf{u}}^{i}, q\right) \frac{f^{i+1}-f^{i}}{\Delta t}=\int_{0}^{T}\left(\nabla \cdot \mathbf{u}^{[r]}, q\right)\left[\left(f^{[r]}\right)_{\Delta t}^{+}\right] d t$.

We then identify the sums as appropriate integrals of piecewise functions on $[0, T]$; thus, (82) becomes:

$$
\begin{aligned}
& \int_{0}^{T}\left(k\left(\nabla \cdot \overline{\mathbf{u}}^{[r]}\right) \nabla p^{[r]}, \nabla q\right) f^{[r]} d t-\int_{0}^{T}\left(\nabla \cdot \overline{\mathbf{u}}^{[r]}, q\right)\left[\left(f^{[r]}\right)_{\Delta t}^{+}\right] d t \\
& \quad=\int_{0}^{T}\left(S^{[r]}, q\right) f^{[r]} d t-\int_{0}^{T}\left\langle\psi^{[r]}, q\right\rangle f^{[r]} d t+\left(\nabla \cdot \mathbf{u}^{0}, q\right) f^{1}-\left(\nabla \cdot \overline{\mathbf{u}}^{r}(T), q\right) f^{r} .
\end{aligned}
$$


Limit passage on the linear terms in both (81) and (82) proceeds exactly as before, using the properties in Definition 4. However, owing to the loss of regularity in the case $\delta=0$, we need to recover an estimate on

$$
\frac{d}{d t} L\left[\nabla \cdot \overline{\mathbf{u}}^{[r]}\right]
$$

to secure limit passage on the nonlinear term (the analogue of Lemma 8).

Lemma 11 For the sequence $\overline{\mathbf{u}}^{[r]} \in \mathbf{V}$ (as in (102)-(104)) such that $\overline{\mathbf{u}}^{[r]} \rightarrow \mathbf{u}$ in $L^{2}(0, T ; \mathbf{V})$, we also have that $\nabla \cdot \overline{\mathbf{u}}^{[r]} \rightarrow \nabla \cdot \mathbf{u}$ in $L^{2}\left(0, T ; L^{2}(\Omega)\right)$.

Proof (of Lemma 11) Consider $q \in V$ :

$$
\left|\left(\frac{d}{d t}\left(L\left[\nabla \cdot \overline{\mathbf{u}}^{i}\right]\right), q\right)_{L^{2}(\Omega)}\right|=\left|\left(\left(\nabla \cdot \overline{\mathbf{u}}^{i}\right)_{\Delta t}, q\right)\right| .
$$

Directly from (83), we see that

$$
\left|\left(\left(\nabla \cdot \overline{\mathbf{u}}^{i}\right)_{\Delta t}, q\right)\right| \leq[\Delta t]\left(\left.\widehat{\kappa}|| p^{i}\left\|_{1}+\right\| \psi^{i}\left\|_{L^{2}\left(I_{D, v}\right)}+\right\| S^{i}\right|_{L^{2}(\Omega)}\right)\|q\|_{V} .
$$

Summing on $i=1, \ldots, r$, we infer that $\frac{d}{d t} L\left[\nabla \cdot \overline{\mathbf{u}}^{[r]}\right] \in L^{2}\left(0, T ; V^{\prime}\right)$ and

$$
\left\|\frac{d}{d t} L\left[\nabla \cdot \mathbf{u}^{[r]}\right]\right\|_{L^{2}\left(0, T ; V^{\prime}\right)} \leq C(\widehat{\kappa}) \cdot\left[\left\|p^{i}\right\|_{L^{2}(0, T ; V)}+\left\|\psi^{i}\right\|_{L^{2}\left(0, T ; L^{2}\left(I_{D, v}\right)\right)}+\left\|S^{i}\right\|_{L^{2}\left(0, T ; L^{2}(\Omega)\right)}\right] .
$$

Thus we have secured the bounds associated with:

$$
\begin{array}{r}
L\left[\nabla \cdot \overline{\mathbf{u}}^{[r]}\right] \in L^{2}\left(0, T ; H^{\varepsilon}(\Omega)\right) \\
\frac{d}{d t} L\left[\nabla \cdot \overline{\mathbf{u}}^{[r]}\right] \in L^{2}\left(0, T ; V^{\prime}\right) .
\end{array}
$$

Again, as in the proof of Lemma 8, we utilize the Aubin-Lions Lemma to guarantee that $\nabla \cdot \overline{\mathbf{u}}^{[r]} \rightarrow \nabla \cdot \mathbf{u}$ in the sense of $L^{2}\left(0, T ; L^{2}(\Omega)\right)$.

At this point, limit passage as $r \rightarrow \infty$ proceeds as in Step 4 in the $\delta>0$ case, and we have that (27)-(28) is satisfied for any $f \in C_{0}^{\infty}(0, T), q \in V$ and $\mathbf{w} \in \mathbf{V}$.

\section{Step 5: Properties of the solution}

The bounds (102)-(104) provide the solution $(p, \mathbf{u})$ with the properties that: $\mathbf{u} \in$ $L^{\infty}([0, T] ; \mathbf{V})$, and thus $\nabla \cdot \mathbf{u} \in L^{\infty}\left([0, T] ; L^{2}(\Omega)\right)$, as well as $p \in L^{2}(0, T ; V)$ (see Section 3.5 for more details). In light of (105) and the bounds in (102)-(104), we also see that the following estimate holds for all $q \in V$ and $f \in C_{0}^{\infty}(0, T)$ :

$$
\begin{aligned}
& \left|\int_{0}^{T}(\nabla \cdot \mathbf{u}, q) f^{\prime} d t\right| \leq \\
& \quad\left[\widehat{\kappa}\|p\|_{L^{2}(0, T ; V)}+\|S\|_{L^{2}\left(0, T ; L^{2}(\Omega)\right)}+\|\psi\|_{L^{2}\left(0, T ; L^{2}\left(\Gamma_{D, v}\right)\right.}\right]\|q\|_{L^{2}(0, T ; V)}\|f\|_{C([0, T])} \\
& \quad+\left(\sup _{[0, T]}\|\mathbf{u}\| \mathbf{v}\right)\|q\|_{V}\|\| f \|_{C([0, T])} .
\end{aligned}
$$


This estimate implies that $\nabla \cdot \mathbf{u}_{t} \in L^{2}\left(0, T ; V^{\prime}\right)$, and by the density of the set $\left\{\nabla q: q \in H_{0}^{1}(\Omega)\right\}$ in $\left(L^{2}(\Omega)\right)^{3}$, we also have (via Stokes' Theorem) that $\mathbf{u}_{t} \in$ $L^{2}\left(0, T ;\left(L^{2}(\Omega)\right)^{3}\right)$. Combining this with the fact that $\mathbf{u} \in L^{2}(0, T ; \mathbf{V})$, we have by [18] that $\mathbf{u} \in C\left(0, T ;\left(L^{2}(\Omega)\right)^{3}\right)$. Additionally, as $\nabla \cdot \mathbf{u}_{t} \in L^{2}\left(0, T ; V^{\prime}\right)$ with $\nabla \cdot \mathbf{u} \in$ $L^{2}\left(0, T ; L^{2}(\Omega)\right) \subset L^{2}\left(0, T ; V^{\prime}\right)$, we know by $\left[18\right.$, p. 302] that $\nabla \cdot \mathbf{u} \in C\left([0, T] ; V^{\prime}\right)$.

By the membership of $\mathbf{u}$ in $L^{2}(0, T ; \mathbf{V})$, taking test functions of the form $\mathbf{w} f(\cdot)$ (as above-which are dense in this space), we may consider $\mathbf{u}$ as a valid test function in (27). However, $\mathbf{u}_{t}$ is not a valid test function for the elasticity equation; thus, a priori estimates on solutions must be handled in the discrete setting and obtained via limit passage. Energy estimates have been detailed in Section 3.5, where the final energy estimate on solutions is shown and (124) results.

\section{Step 6: Recovering the initial condition}

We follow [55] to recover the initial condition starting from the pressure equation (28). For any $q \in V$, we can define

$$
\begin{aligned}
G(t) & \equiv(\nabla \cdot \mathbf{u}(t), q) \\
H(t) & \equiv-(k(\nabla \cdot \mathbf{u}) \nabla p, \nabla q)-\langle\psi, q\rangle_{\Gamma_{D, v}}+(S, q) \\
F(t) & \equiv \int_{0}^{t} H(\tau) d \tau
\end{aligned}
$$

and note that $F(t)$ is absolutely continuous on $[0, T]$ with $F^{\prime}(t)=H(t)$ a.e. $(0, T)$.

Utilizing these definitions in (28) and performing integration by parts, for all $f \in C_{0}^{\infty}(0, T)$ we obtain

$$
\int_{0}^{T}\left(G(t) f^{\prime}(t)+F^{\prime}(t) f(t)\right) d t=\int_{0}^{T}\left((G(t)-F(t)) f^{\prime}(t)\right) d t=0 .
$$

Thus $G$ and $F$ differ by a constant: $G-F=c$.

We return to (105) and consider $f \in C^{\infty}([0, T])$ with $f(0)=1$ and $f(T)=0$; completing the limit passage here we see that for such $f$ :

$$
\begin{aligned}
\int_{0}^{T}(k(\nabla \cdot \mathbf{u}) \nabla p, \nabla q) f d t-\int_{0}^{T}(\nabla \cdot \mathbf{u}, q) f^{\prime} d t \\
=\int_{0}^{T}(S, q) f d t-\int_{0}^{T}\langle\psi, q\rangle_{\Gamma_{D, v}} f d t+\left(\nabla \cdot \mathbf{u}^{0}, q\right)
\end{aligned}
$$

for all $q \in V$. This can be rewritten as

$$
\int_{0}^{T} H f d t+\int_{0}^{T} G f^{\prime}=-\left(\nabla \cdot \mathbf{u}^{0}, q\right) .
$$

Integrating by parts, we have:

$$
\int_{0}^{T} H f d t-\int_{0}^{T} F^{\prime} f d t+\left.[(F(t)+c) f(t)]\right|_{0} ^{T}=-\left(\nabla \cdot \mathbf{u}^{0}, q\right) .
$$

Then it follows (by choosing $T=0$ ) that $c=\left(\nabla \cdot \mathbf{u}^{0}, q\right)$. Identifying $F^{\prime}=H$ a.e. and since $G=F+c$, we have

$$
(\nabla \cdot \mathbf{u}(t), q)-\left(\nabla \cdot \mathbf{u}^{0}, q\right)=\int_{0}^{T}\left[-(k(\nabla \cdot \mathbf{u}) \nabla p, \nabla q)-\langle\psi, q\rangle_{\Gamma_{D, v}}+(S, q)\right] d t,
$$


for all $q \in V$ and a.e. $(0, T)$. This implies that the initial condition $\nabla \cdot \mathbf{u}(0)=$ $\nabla \cdot \mathbf{u}^{0}=d_{0}$ is satisfied for solutions to (27)-(28).

This completes the proof of Theorem 2.

\subsection{A priori estimates}

The energy estimates derived in this section are attained in two different ways depending on the parameter $\delta$. For $\delta>0$, the estimates are obtained by utilizing $\mathbf{u}, \mathbf{u}_{t}$, and $p$ as test functions in (25)-(26). Utilizing $\mathbf{u}$ and $\mathbf{u}_{t}$ (in the appropriate sense) as test functions for $\delta>0$ is functionally justified after the solutions have been constructed; this is not the case for $\delta=0$, as $a\left(\mathbf{u}, \mathbf{u}_{t}\right)$ cannot be written with $\mathbf{u} \in L^{2}(0, T ; \mathbf{V})$ and $\mathbf{u}_{t} \in L^{2}\left(0, T ; \mathbf{V}^{\prime}\right)$ only. Hence, for $\delta=0$ we use a priori estimates on the discrete solutions $\left(p^{i}, \mathbf{u}^{i}\right) \in V \times \mathbf{V}$ to (27)-(28) and then pass to the limit.

\subsubsection{Estimates for $\delta>0$}

Thanks to the regularity of constructed solutions, the calculations below hold in the appropriate functional setting (not just in the sense of distributions).

\subsubsection{Energy identities: $\delta>0$}

Using the test functions $\mathbf{u}, \mathbf{u}_{t}$, and $p$ in (25)-(26), we obtain the following formal identities:

$$
\begin{aligned}
2 E(\mathbf{u})+\delta \frac{d}{d t} E(\mathbf{u})-(p, \nabla \cdot \mathbf{u}) & =-(\mathbf{F}, \mathbf{u})+\langle\mathbf{g}, \mathbf{u}\rangle_{\Gamma_{N}} \\
\frac{d}{d t} E(\mathbf{u})+2 \delta E\left(\mathbf{u}_{t}\right)-\left(p, \nabla \cdot \mathbf{u}_{t}\right) & =-\left(\mathbf{F}, \mathbf{u}_{t}\right)+\left\langle\mathbf{g}, \mathbf{u}_{t}\right\rangle_{\Gamma_{N}} \\
\left(\nabla \cdot \mathbf{u}_{t}, p\right)+E(p) & =(S, p)-\langle\psi, p\rangle_{\Gamma_{D, v}} .
\end{aligned}
$$

Using Assumption 3.1 and a combination of trace theorem, Young's inequality, and Gronwall's inequality, we obtain the a priori estimate:

$$
E(\mathbf{u}(t)) \leq\left[C_{1} E(\mathbf{u}(0))+\left.\left(\frac{C_{1}}{1+\delta}\right) \operatorname{DATA}_{\delta}\right|_{0} ^{t}\right] \exp \left(\frac{C_{2} t}{1+\delta}\right) .
$$

Immediately from (120) it follows that

$$
\int_{0}^{T} E(\mathbf{u}) d t \leq\left[C_{1} E(\mathbf{u}(0))+\left.\left(\frac{C_{1}}{1+\delta}\right) \operatorname{DATA}_{\delta}\right|_{0} ^{T}\right] \frac{1+\delta}{C_{2}}\left[\exp \left(\frac{C_{2} T}{1+\delta}\right)-1\right],
$$

and finally

$$
\begin{aligned}
\sup _{t \in[0, T]} E(\mathbf{u}(t))+\int_{0}^{T}\left[E(p(t))+E(\mathbf{u}(t))+E\left(\mathbf{u}_{t}\right)\right] d t & \\
\leq & C_{1}\left[E(\mathbf{u}(0))+\left.\left(\frac{1}{1+\delta}\right) \operatorname{DATA}_{\delta}\right|_{0} ^{T}\right] \exp \left(\frac{C_{2} T}{1+\delta}\right) .
\end{aligned}
$$


3.5.3 Estimates for $\delta=0$

In what follows below, we may utilize $\mathbf{u} \in L^{2}(0, T ; \mathbf{V})$ and $p \in L^{2}(0, T ; V)$ as valid test functions. We cannot, however, utilize $\mathbf{u}_{t} \in L^{2}\left(0, T ; \mathbf{V}^{\prime}\right)$ as a test function on (27). The final a priori estimates on solutions are justified by considering solutions (discretized in time) to (81)-(83), and completing the limit passage as in Step 4 of Section 3.4.2 after making the appropriate calculations. Note that this will yield cancellation of the terms involving $\left(p^{[r]},\left(\nabla \cdot \mathbf{u}^{[r]}\right)_{\Delta t}\right)$, but in the limit passage we will obtain only inequalities.

\subsubsection{Energy identities: $\delta=0$}

By testing equation (28) with $p$, integrating in time we obtain:

$$
\int_{0}^{T}\left(\nabla \cdot \mathbf{u}_{t}, p\right)_{\left(V^{\prime}, V\right)} d t+\int_{0}^{T} E(p) d t=\int_{0}^{T}\left[(S, p)-\langle\psi, p\rangle_{\Gamma_{D, v}}\right] d t .
$$

By testing equation (27) with $\mathbf{u}$ and integrating in time we obtain:

$$
2 \int_{0}^{T} E(\mathbf{u}) d t=\int_{0}^{T}(p, \nabla \cdot \mathbf{u}) d t+\int_{0}^{T}\left[\langle\mathbf{g}, \mathbf{u}\rangle_{\Gamma_{N}}-(\mathbf{F}, \mathbf{u})\right] d t .
$$

\subsubsection{Final estimate: $\delta=0$}

Consider the discrete pre-Grownall estimate in (99) applied to the discretized solution $\left(\bar{p}^{i}, \overline{\mathbf{u}}^{i}\right)$. Utilizing Gronwall's lemma, identifying sums up to $r$ with integrals of $\left(p^{[r]}, \mathbf{u}^{[r]}\right) \in V \times \mathbf{V}$, and using the weak lower semi-continuity of the norm, we have our final a priori estimate on $[0, T]$ in the $\delta=0$ case:

$$
\sup _{t \in[0, T]} E(\mathbf{u}(t))+\int_{0}^{T}[E(p(t))+E(\mathbf{u}(t))] d t \leq C_{1}\left[E(\mathbf{u}(0))+\left.\mathrm{DATA}_{0}\right|_{0} ^{T}\right] e^{C_{2} T}
$$

Remark 20 (A Priori Estimate: A Stronger Solution for $\delta=0$ ) Let us formally admit $\mathbf{u}_{t}$ and $p_{t}$ as test functions in (27) and (28). In the case of constant permeability, this yields estimates for "strong" solutions as in [40,51]. The key difference in this case is the structure of the nonlinear term which does not allow pointwise control of the pressure. The "formal identity" below follows from differentiating (15) with $\delta=0$ and utilizing the test functions $\mathbf{u}_{t}$ and $p_{t}$ :

$$
2 E\left(\mathbf{u}_{t}(t)\right)+\frac{1}{2} \int_{\Omega} k(\nabla \cdot \mathbf{u}) \frac{d}{d t}\left(|\nabla p|^{2}\right) d \Omega=\left(\mathbf{F}, \mathbf{u}_{t}\right)+\left\langle\mathbf{g}, \mathbf{u}_{t}\right\rangle_{\Gamma_{N}}-\left\langle\psi, p_{t}\right\rangle_{\Gamma_{D, v}} .
$$

3.6 Sharp estimates (with respect to constants)

In this section we present the estimates obtained above with specific control of the constants associated with permeability, Poincare's inequality, Korn's inequality, 
trace theorem, and Young's inequality. Recall the system (15)-(16) from Section 3. We adjust the notation to be:

$$
\begin{aligned}
E_{e}(\mathbf{u}(t)) & =\frac{1}{2}\left[\lambda_{e}\|\nabla \cdot \mathbf{u}(t)\|^{2}+2 \mu_{e}\|\nabla \mathbf{u}\|^{2}+2 \mu_{e}\left(\nabla \mathbf{u}, \nabla \mathbf{u}^{T}\right)\right] \\
E_{v}(\mathbf{u}(t)) & =\frac{1}{2}\left[\lambda_{v}\|\nabla \cdot \mathbf{u}(t)\|^{2}+2 \mu_{v}\|\nabla \mathbf{u}\|^{2}+2 \mu_{v}\left(\nabla \mathbf{u}, \nabla \mathbf{u}^{T}\right)\right] \\
E(p(t)) & =(k(\nabla \cdot \mathbf{u}) \nabla p, \nabla p) .
\end{aligned}
$$

We note the following inequalities:

$$
\begin{array}{rlrl}
\|p\|_{1} \leq \frac{C(P)}{\kappa} E(p), & & \|\mathbf{u}\|_{1} \leq C(K) E(\mathbf{u}) \\
\operatorname{tr}[\mathbf{u}] \leq C(\gamma)\|\mathbf{u}\|_{1}, & \operatorname{tr}[p] \leq C(\gamma)\|p\|_{1},
\end{array}
$$

where $C(P)$ denotes the Poincaré constant, $C(K)$ denotes the constant associated with Korn's inequality, and $C(\gamma)$ denotes the constant associated with the trace theorem.

Lemma 12 Let $\delta>0$. Then we have the estimate:

$$
\begin{gathered}
\sup _{t \in[0, T]}\left[E_{e}(\mathbf{u}(t))+\delta E_{v}(\mathbf{u}(t))\right]+\int_{0}^{T}\left[E_{e}(\mathbf{u})+\delta E_{v}\left(\mathbf{u}_{t}\right)\right] d t+\int_{0}^{T} E(p) d t \\
\leq \mathscr{C}\left[e^{\mathscr{K}_{1} T}+e^{\mathscr{K}_{2} T}\right]
\end{gathered}
$$

where

$$
\begin{aligned}
\mathscr{C} \equiv & {\left[C E_{e}(\mathbf{u}(0))+\delta C E_{v}(\mathbf{u}(0))\right] } \\
& +C\left(\gamma, P, \kappa^{-1}\right) \int_{0}^{T}\left[\|\mathbf{F}\|_{0}^{2}+\|\mathbf{g}\|_{L^{2}\left(\Gamma_{N}\right)}^{2}+\|S\|_{0}^{2}+\|\psi\|_{L^{2}\left(\Gamma_{D, v}\right)}^{2}\right] \\
\mathscr{K}_{1} \equiv & C\left(\gamma, K, \mu_{e}, \lambda_{e}\right) \\
\mathscr{K}_{2} \equiv & C\left(\gamma, K, \mu_{v}, \lambda_{v}, \delta^{-1}\right) .
\end{aligned}
$$

Lemma 13 Let $\delta=0$. Then we have the estimate:

$$
\sup _{t \in[0, T]} E_{e}(\mathbf{u}(t))+\int_{0}^{T}\left[E(p)+E_{e}(\mathbf{u})\right] d t \leq \mathscr{C} e^{\mathscr{K} T}
$$

where

$$
\begin{aligned}
\mathscr{C} \equiv & C\left(\gamma, K, \mu_{e}, \lambda_{e}\right) E_{e}(\mathbf{u}(0)) \\
& +C\left(\gamma, K, \mu_{e}, \lambda_{e}\right) \sup _{[0, T]}\left(\|\mathbf{g}(t)\|_{L^{2}\left(\Gamma_{N}\right)}^{2}+\|\mathbf{F}(t)\|_{0}^{2}\right) \\
& +C\left(\gamma, P, \kappa^{-1}\right) \int_{0}^{T}\left(\|\mathbf{g}\|_{L^{2}\left(\Gamma_{N}\right)}^{2}+\left\|\mathbf{g}_{t}\right\|_{L^{2}\left(\Gamma_{N}\right)}^{2}+\|\psi\|_{L^{2}\left(\Gamma_{D, v}\right)}^{2}\right) \\
& +C\left(\gamma, P, \kappa^{-1}\right) \int_{0}^{T}\left(\|\mathbf{F}\|_{0}^{2}+\left\|\mathbf{F}_{t}\right\|_{0}^{2}+\|S\|_{0}^{2}\right)
\end{aligned}
$$

and

$$
\mathscr{K} \equiv C\left(\gamma, K, P, \mu_{e}, \lambda_{e}, \kappa^{-1}\right) .
$$




\section{Numerical study}

In this section we perform a numerical study of one-dimensional poro-elastic and poro-visco-elastic models to investigate how the data regularity given in Definition 3 influences the theoretical estimates obtained in Section 3.6.

Various numerical approaches have been proposed for the solution of poroelastic models, whereas less attention has been devoted to the poro-visco-elastic case. Time discretization is typically performed via a Backward Euler method; spatial discretization has been addressed by means of various techniques, including finite difference schemes $[23,24]$ and finite element methods. Within the context of finite element methods, two main approaches have been proposed. The first approach is a two-field formulation of the problem in which the pair $(\mathbf{u}, p)$ is approximated using the Taylor-Hood finite element space [45]. The second approach is a four-field formulation emanating from a least-squares variational principle in which, together with the original pair $(\mathbf{u}, p)$, also the stress $\mathbf{T}$ and the Darcy fluid velocity $\mathbf{v}$ are treated as independent variables of the problem. In the four-field formulation, the Taylor-Hood finite element space is still used to approximate $\mathbf{u}$ and $p$, whereas the Raviart-Thomas finite element space $[4,46,48]$ is utilized to approximate the pair $(\mathbf{T}, \mathbf{v})$. We refer to [55] for a theoretical analysis of the first approach and to [31] for a description of the implementation of both approaches and a comparison in the solution of several benchmark case studies in plane strain conditions. We also refer to $[41,42]$ for another finite element approach in which the Raviart-Thomas finite element space for the approximation of $\mathbf{v}$ and $p$ is coupled with a Discontinuous Galerkin finite element formulation to treat the elastic part of the Biot model in the incompressible limit.

In the present article, we adopt the Backward Euler scheme for time advancement and the four-field finite element approach for spatial discretization. Our formulation is an extension of the four-field method based on the use of dual mixed hybridized finite elements $($ see $[2,4]$ ), with the addition of a solid pressure parameter to weakly enforce the dependence of the material porosity on the divergence of the solid displacement (see also [9]). The four-field approach is adopted to properly compute the gradients involved in the energy estimates; a hybridization procedure is used to reduce the number of degrees of freedom involved in the numerical computations. Our scheme is illustrated and implemented in the one-dimensional case to allow, on the one hand, a preliminary validation against analytical solutions in both linear and nonlinear models, and, on the other hand, to perform a tractable and immediate verification of the theoretical energy estimates obtained in Section 3.6 as a function of time regularity of problem data. The convergence analysis of the numerical scheme and its extension to multiple spatial dimensions go beyond the scope of the present article and are currently object of an ongoing research activity.

\subsection{The one-dimensional model}

We consider the nonlinear boundary value/initial value problem (15)-(22) from Section 2 in the computational domain $\Omega=\left(x_{\text {start }}, x_{\text {end }}\right)$ of length $L=x_{\text {end }}-x_{\text {start }}$ with boundary $\partial \Omega=\left\{x_{\text {start }}, x_{\text {end }}\right\}$ and outward unit normal vector $n$ such that $n\left(x_{\text {start }}\right)=-1$ and $n\left(x_{\text {end }}\right)=+1$. We also define the computational time domain 
$t \in\left(t_{\text {start }}, t_{\text {end }}\right)$ of length $T=t_{\text {end }}-t_{\text {start }}$, in such a way that the one-dimensional (1D) system to be solved in the space-time domain $\mathscr{Q}_{T}:=\Omega \times\left(t_{\text {start }}, t_{\text {end }}\right)$ is:

$$
\begin{aligned}
\frac{\partial \sigma}{\partial x} & =-F, \\
\frac{\partial \zeta}{\partial t}+\frac{\partial v}{\partial x} & =S,
\end{aligned}
$$

with the constitutive equations:

$$
\begin{aligned}
\sigma & =2 \mu_{e} \frac{\partial u}{\partial x}-\wp+\delta \frac{\partial}{\partial t}\left[2 \mu_{v} \frac{\partial u}{\partial x}-\frac{\lambda_{v}}{\lambda_{e}} \wp\right]-p \\
\frac{\wp}{\lambda_{e}}+\frac{\partial u}{\partial x} & =0 \\
\zeta & =-\frac{\wp}{\lambda_{e}} \\
v & =-k\left(-\frac{\wp}{\lambda_{e}}\right) \frac{\partial p}{\partial x} .
\end{aligned}
$$

Throughout this section, we use the symbol $\sigma$ to indicate the one-dimensional analogue of the total stress tensor $\mathbf{T}$ defined in Equations (4)-(5). System (138)(143) must be completed by suitable initial and boundary conditions. Similarly to the general case described in Section 2, we prescribe

$$
u\left(x, t_{\text {start }}\right)=u^{0}(x) \quad \forall x \in \Omega,
$$

and we consider the following sets of boundary conditions:

$$
\begin{array}{cc}
\sigma n(x, t)=g(x, t), \quad v(x, t) n(x)=0 & \forall x \in \Gamma_{N}, \forall t \in\left(t_{\text {start }}, t_{\text {end }}\right), \\
u(x, t)=0, \quad p(x, t)=0 & \forall x \in \Gamma_{D, p}, \forall t \in\left(t_{\text {start }}, t_{\text {end }}\right), \\
u(x, t)=0, \quad v(x, t) n(x)=\psi(x, t) & \forall x \in \Gamma_{D, v}, \forall t \in\left(t_{\text {start }}, t_{\text {end }}\right) .
\end{array}
$$

Note that $\Gamma_{N} \cup \Gamma_{D, p} \cup \Gamma_{D, v}=\partial \Omega=\left\{x_{\text {start }}, x_{\text {end }}\right\}$, and that $\Gamma_{N}, \Gamma_{D, p}$ and $\Gamma_{D, v}$ can be empty (but not all of them simultaneously). Two differences appear by comparing the $1 \mathrm{D}$ equations (138)-(143) with the multi-dimensional version (15)-(22). The first difference is that the Lamé and viscous parameters are not scaled to unity as to maintain the physical parameters of the problem. The second difference is the introduction of the elastic pressure parameter $\&$ that can be replaced in (9) to write the porosity as

$$
\phi=\phi_{0}-\frac{\mathscr{P}}{\lambda} .
$$

The use of (148) in (7) and (8) allows to evaluate the permeability without explicitly computing the derivative of the displacement field, thereby avoiding the well-known degradation of the accuracy associated with numerical differentiation (see [44], Chapt. 8). This aspect is treated in Section 4.3. Interestingly, the variable $\wp$ is widely utilized in computational mechanics as it serves as Lagrange multiplier to enforce material incompressibility (see [25]). Mathematically, this amounts to a robust numerical treatment of the limit $\lambda_{e} \rightarrow+\infty$ and allows to 
avoid the occurrence of the locking phenomenon in the finite element discretization (see [27] in the case of linear elasticity and Stokes flow). Volumetric locking also affects the numerical treatment of poroelastic models. We refer to [41,42] for a numerical approach to overcome locking based on the combined use of Discontinuous Galerkin and mixed finite elements. Notice also that no boundary conditions are required for the elastic pressure parameter $\wp$ because the total stress is already prescribed on $\Gamma_{N}$ in (145).

\subsection{Numerical algorithm}

The numerical algorithm for the solution of the 1D problem described above is composed by three main steps: (i) temporal semi-discretization; (ii) fixed-point iteration; and (iii) dual mixed hybridized finite element approximation. The details of each step are given in following subsections.

\subsubsection{Temporal semi-discretization}

We divide $\left[t_{\text {start }}, t_{\text {end }}\right]$ into a finite number $r \geq 1$ of subintervals $\left[t_{i-1}, t_{i}\right], i=1, \ldots, r$ of uniform length $\Delta t=T / r$, as in Sections 3.4.1 and 3.4.2. For any smooth function (in time) $\mathscr{W}=\mathscr{W}(x, t)$, we let $\mathscr{W}^{i}:=\mathscr{W}\left(x, t_{i}\right), i=0, \ldots, r$; otherwise, should $\mathscr{W}$ be discontinuous (in time) at $t=t_{i}, i \in[1, r]$, we let $\mathscr{W}^{i}:=\mathscr{W}\left(x, t_{i}^{-}\right), i=1, \ldots, r$. We note that these definitions agree with those introduced in Sections 3.4.2 (functions with $H^{1}$-time regularity) and 3.4.1 (functions with $L^{2}$-time regularity). Using the Backward Euler (BE) method for the time discretization, we are led to the solution of the following sequence of $r$ nonlinearly coupled boundary value problems: Given $u^{i}$ and $\wp^{i}, i=0, \ldots, r-1$, solve:

$$
\begin{aligned}
\frac{\partial \sigma^{i+1}}{\partial x}= & -F^{i+1} \\
\sigma^{i+1}= & 2 \mu_{e} \frac{\partial u^{i+1}}{\partial x}-\wp^{i+1}-p^{i+1}+\delta \frac{1}{\Delta t}\left[2 \mu_{v} \frac{\partial u^{i+1}}{\partial x}-\frac{\lambda_{v}}{\lambda_{e}} \wp^{i+1}\right] \\
& -\delta \frac{1}{\Delta t}\left[2 \mu_{v} \frac{\partial u^{i}}{\partial x}-\frac{\lambda_{v}}{\lambda_{e}} \wp^{i}\right] \\
\frac{\wp^{i+1}}{\lambda_{e}}+\frac{\partial u^{i+1}}{\partial x}= & 0 \\
-\frac{\wp^{i+1}}{\lambda_{e} \Delta t}+\frac{\partial v^{i+1}}{\partial x}= & S^{i+1}-\frac{\wp^{i}}{\lambda_{e} \Delta t}, \\
v^{i+1}= & -k\left(-\frac{\wp^{i+1}}{\lambda_{e}}\right) \frac{\partial p^{i+1}}{\partial x}
\end{aligned}
$$

for $x$ in $\Omega$, with

$$
\begin{array}{rcl}
\sigma^{i+1} n=g^{i+1} & v^{i+1} n=0 & \text { on } \Gamma_{N} \\
u^{i+1}=0 & p^{i+1}=0 & \text { on } \Gamma_{D, p} \\
u^{i+1}=0 & v^{i+1} n=\psi^{i+1} & \text { on } \Gamma_{D, v} .
\end{array}
$$




\subsubsection{Fixed-point iteration}

We adopt a Picard iteration to numerically deal with the nonlinear dependence of the permeability $k$ on $-\wp / \lambda_{e}$ in (153). This approach is similar to that used in [8]. Given $u^{(0)}=u^{i}$ and $\wp^{(0)}=\wp^{j}$, for each $j \geq 0$ until convergence, solve:

$$
\begin{aligned}
& \frac{\partial \sigma^{(j+1)}}{\partial x}=-F^{i+1} \\
& \sigma^{(j+1)}=2 \mu_{e} \frac{\partial u^{(j+1)}}{\partial x}-\wp^{(j+1)}-p^{(j+1)}+\delta \frac{1}{\Delta t}\left[2 \mu_{v} \frac{\partial u^{(j+1)}}{\partial x}-\frac{\lambda_{v}}{\lambda_{e}} \wp^{(j+1)}\right] \\
&-\delta \frac{1}{\Delta t}\left[2 \mu_{v} \frac{\partial u^{i}}{\partial x}-\frac{\lambda_{v}}{\lambda_{e}} \wp^{i}\right] \\
& \frac{\wp^{(j+1)}}{\lambda_{e}}+\frac{\partial u^{(j+1)}}{\partial x}= \\
&-\frac{\wp^{(j+1)}}{\lambda_{e} \Delta t}+\frac{\partial v^{(j+1 / 2)}}{\partial x}= \\
& v^{i+1}-\frac{\wp^{j}}{\lambda_{e} \Delta t}, \\
&=-k\left(-\frac{\wp^{(j)}}{\lambda_{e}}\right) \frac{\partial p^{(j+1)}}{\partial x}
\end{aligned}
$$

for $x$ in $\Omega$, with

$$
\begin{array}{rcl}
\sigma^{(j+1)} n=g^{i+1} & v^{(j+1)} n=0 & \text { on } \Gamma_{N} \\
u^{(j+1)}=0 & p^{(j+1)}=0 & \text { on } \Gamma_{D, p} \\
u^{(j+1)}=0 & v^{(j+1)} n=\psi^{i+1} & \text { on } \Gamma_{D, v} .
\end{array}
$$

The boxed term in (162) characterizes the adopted Picard iteration, where the permeability at the iteration level $j+1$ is computed using the previously available elastic pressure $\wp^{(j)}$. The algorithm described above is a (semi-implicit) variant of the staggered (or loosely coupled) algorithm proposed and successfully utilized in [9] for the numerical study of a problem similar to that considered in this work. It is well known that the use and analysis of solution algorithms for the treatment of solid-fluid interacting problems is a nontrivial subject and would require a deeper investigation. Since such an investigation is not the main focus of this article, we postpone the examination of different solution maps to a future research.

\subsection{The Dual Mixed Hybridized (DMH) finite element discretization}

The choice of a suitable spatial discretization is a crucial and extremely delicate issue for the problem at hand. This is due to the fact that our numerical study aims at interpreting the theoretical estimates obtained in Section 3.6 which require the evaluation of gradient quantities under different regularity conditions (in time) of input data. Thus, it is extremely important to approximate gradients accurately. 
It is well-known that numerical differentiation is a very delicate process usually affected by a degradation in the approximation accuracy (see, e.g., [44] Chapters 8 and 10). For this reason, the use of a dual mixed method where the dual variables (the total stress $\sigma$ and the discharge velocity $v$ ) are treated as independent variables as well as the primal unknowns (the solid displacement $u$ and the fluid pressure $p$ ) appears to be a better option compared to a displacement-based method where the sole primal variables are directly discretized.

In particular, we propose here a dual mixed hybridized (DMH) finite element method which generalizes to the poro-elastic and poro-visco-elastic cases the approach proposed in [20,19] for linear incompressible elasticity and Stokes equations. We adopt the lowest-order Raviart-Thomas (RT) finite element pair [46] for the dual and primal variables which provides:

i) equal-order optimal accuracy for the approximation of the pairs $\sigma, u$ and $v, p$ in the graph norm of the space $H(\operatorname{div}, \Omega) \times L^{2}(\Omega)$, where

$$
H(\operatorname{div}, \Omega):=\left\{\tau: \Omega \rightarrow \mathbb{R} \mid \tau \in L^{2}(\Omega), \frac{\partial \tau}{\partial x} \in L^{2}(\Omega)\right\}
$$

(coinciding with $H^{1}(\Omega)$ in the 1D case);

i) exact satisfaction of self-equilibrium at each element level;

iii) exact satisfaction of the action-reaction principle at the discrete level for each internal and boundary interelement;

iv) weak satisfaction of Dirichlet boundary conditions.

To overcome the limitation in (iv) and to substantially reduce the computational effort, we resort, in coding, to the hybridization technique (see [48]) that makes (in 1D) the DM-RT method completely equivalent to a standard nodal displacement formulation (for more details on hybridization, we refer to $[2,4]$ ).

\subsubsection{Finite element spaces}

Let $h>0$ be the spatial discretization parameter. We introduce the family of triangulations $\left\{\mathscr{T}_{h}\right\}_{h>0}$ defined for each $h$ as the partition of $\Omega$ into subintervals $K_{k}=\left(x_{k-1}, x_{k}\right), k=1, \ldots, \mathscr{K}_{h}, \mathscr{K}_{h} \geq 1$, in such a way that $\cup_{K_{k} \in \mathscr{T}_{h}} K_{k}=\Omega$. On each $K_{k}$ we denote by $\partial K_{k}$ the boundary of the interval and associate with $\partial K_{k}$ the unit normal vector $n_{k}$ such that $n_{k}=-1$ at $x=x_{k-1}$ and $n_{k}=+1$ at $x=x_{k}$. The length of $K_{k}$ is $h_{k}$ and we set $h:=\max _{K_{k} \in \mathscr{T}_{h}} h_{k}$. For a given integer $q \geq 0$ we denote by $\mathbb{P}_{q}\left(K_{k}\right)$ the set of polynomials of degree $\leq q$ defined on $K_{k}$. Let us define the following finite element spaces:

$$
\begin{aligned}
U_{h} & =\left\{u_{h} \in L^{2}(\Omega) \text { such that } u_{h} \in \mathbb{P}_{0}\left(K_{k}\right) \forall K_{k} \in \mathscr{T}_{h}\right\}, \\
V_{h} & =\left\{j_{h} \in L^{2}(\Omega) \text { such that } j_{h} \in \mathbb{P}_{1}\left(K_{k}\right) \forall K_{k} \in \mathscr{T}_{h}\right\}, \\
M_{h} & =\left\{\mu_{h} \in \mathbb{R} \text { such that }\left|\mu_{k}\right|<+\infty \forall x_{k} \in \mathscr{T}_{h}\right\} .
\end{aligned}
$$

Moreover, to account for Dirichlet boundary conditions, we introduce the following subspaces of $M_{h}$ :

$$
\begin{gathered}
M_{h, 0}^{u}=\left\{\mu_{h} \in M_{h} \text { such that } \mu_{h}=0 \text { on } \Gamma_{D}\right\}, \\
M_{h, 0}^{p}=\left\{\mu_{h} \in M_{h} \text { such that } \mu_{h}=0 \text { on } \Gamma_{D, p}\right\},
\end{gathered}
$$


where $\Gamma_{D}=\Gamma_{D, p} \cup \Gamma_{D, v}$. Let $\mathbf{U}_{h}:=\left[\sigma_{h}, u_{h}, \widehat{u}_{h}\right]^{T}$ and $\mathbf{P}_{h}:=\left[v_{h}, p_{h}, \widehat{p}_{h}\right]^{T}$ denote the discrete elastic and fluid variables.

Let also $\mathscr{U}_{h}^{u}:=V_{h} \times U_{h} \times M_{h, 0}^{u}$ and $\mathscr{U}_{h}^{p}:=V_{h} \times U_{h} \times M_{h, 0}^{p}$ be the finite element spaces for the triplets $\mathbf{U}_{h}$ and $\mathbf{P}_{h}$, respectively. The pairs $\sigma_{h}, u_{h}$ (resp., $v_{h}, p_{h}$ ) are the approximation of $\sigma, u$ (resp., $v, p$ ) in the interior of each element $K_{k} \in \mathscr{T}_{h}$. The variables $\widehat{u}_{h}$ (resp., $\widehat{p}_{h}$ ) are the approximation of $u$ (resp., $p$ ) at each node of $\mathscr{T}_{h}$. The fundamental property of $\widehat{u}_{h}$ (resp., $\widehat{p}_{h}$ ) is that they are single-valued at each node $x_{k}, k=0, \ldots, \mathscr{K}_{h}$ whereas $u_{h}$ (resp., $p_{h}$ ) experience finite jump discontinuities at each node. As shown below, the variables $\widehat{u}_{h}$ (resp., $\widehat{p}_{h}$ ) are the Lagrange multipliers of the continuity constraint of $\sigma_{h}$ (resp., $v_{h}$ ) at each internal node $x_{k}$, $k=1, \ldots, \mathscr{K}_{h}-1$. The dual-mixed hybridized finite element approximation of (158)- (166) is:

Find $\left(\mathbf{U}_{h}, \wp_{h}, \mathbf{P}_{h}\right) \in\left(\mathscr{U}_{h}^{u} \times U_{h} \times \mathscr{U}_{h}^{p}\right)$ such that:

$$
\begin{aligned}
& A\left(m_{u}^{-1} \sigma_{h}, \tau_{h}\right)+B\left(u_{h}, \tau_{h}\right)-C\left(\widehat{u}_{h}, \tau_{h}\right)+\frac{m_{p}}{m_{u}} D\left(\wp_{h}, \tau_{h}\right) \\
& +\frac{1}{m_{u}} D\left(p_{h}, \tau_{h}\right)=q_{h}^{i} \quad \forall \tau_{h} \in V_{h} \\
& \frac{1}{\lambda_{e}}\left(\wp_{h}, \xi_{h}\right)_{h}+G\left(\xi_{h}, \widehat{u}_{h}\right)=0 \quad \forall \xi_{h} \in U_{h} \\
& B\left(\xi_{h}, \sigma_{h}\right)=-\left(F^{i+1}, \xi_{h}\right)_{h} \quad \forall \xi_{h} \in U_{h} \\
& C\left(\mu_{h}, \sigma_{h}\right)=\left.g^{i+1} \mu_{h}\right|_{\Gamma_{N}} \quad \forall \mu_{h} \in M_{h, 0}^{u} \\
& A\left(k^{-1} v_{h}, \tau_{h}\right)-B\left(p_{h}, \tau_{h}\right)+C\left(\widehat{p}_{h}, \tau_{h}\right)=0 \quad \forall \tau_{h} \in V_{h} \\
& -\frac{1}{\lambda_{e} \Delta t}\left(\wp_{h}, \xi_{h}\right)_{h}+B\left(\xi_{h}, v_{h}\right)=\left(S^{i+1}, \xi_{h}\right)_{h}-\frac{1}{\lambda_{e} \Delta t}\left(\wp_{h}^{i}, \xi_{h}\right)_{h} \quad \forall \xi_{h} \in U_{h} \\
& C\left(\mu_{h}, v_{h}\right)=\left.\psi^{i+1} \mu_{h}\right|_{D, v} \quad \forall \mu_{h} \in M_{h, 0}^{p}
\end{aligned}
$$

where:

$$
\begin{array}{r}
m_{u}:=2\left(\mu_{e}+\delta \mu_{v} / \Delta t\right), \quad m_{p}:=\left(1+\delta \lambda_{v} /\left(\Delta t \lambda_{e}\right)\right) \\
(f, g)_{h}:=\sum_{K_{k} \in \mathscr{T}_{h}} \int_{K_{k}} f g d x, \\
q_{h}^{i}:=\frac{\delta}{\Delta t} \frac{H_{V}}{\lambda_{e}} \frac{1}{m_{u}} D\left(\wp_{h}^{i}, \tau_{h}\right), \quad H_{V}:=\lambda_{v}+2 \mu_{v},
\end{array}
$$


and the bilinear forms $A, B, C, D$ and $G$ are defined as:

$$
\begin{aligned}
A\left(m_{u}^{-1} J_{h}, \tau_{h}\right) & =\sum_{K_{k} \in \mathscr{T}_{h}} \int_{K_{k}} m_{u}^{-1} J_{h} \tau_{h} d x & \forall\left(J_{h}, \tau_{h}\right) \in\left(V_{h} \times V_{h}\right) \\
B\left(q_{h}, J_{h}\right) & =\sum_{K_{k} \in \mathscr{T}_{h}} \int_{K_{k}} q_{h} \frac{\partial J_{h}}{\partial x} d x & \forall\left(q_{h}, J_{h}\right) \in\left(U_{h} \times V_{h}\right) \\
C\left(\mu_{h}, J_{h}\right) & =\sum_{K_{k} \in \mathscr{T}_{h}} \int_{\partial K_{k}} \mu_{h} J_{h} n_{k} d s & \forall\left(\mu_{h}, J_{h}\right) \in\left(M_{h} \times V_{h}\right) \\
D\left(q_{h}, \tau_{h}\right) & =\sum_{K_{k} \in \mathscr{T}_{h}} \int_{K_{k}} q_{h} \tau_{h} d x & \forall\left(q_{h}, \tau_{h}\right) \in\left(U_{h} \times V_{h}\right) \\
G\left(\mu_{h}, \xi_{h}\right) & =\sum_{K_{k} \in \mathscr{T}_{h}} \int_{\partial K_{k}} \xi_{h} \mu_{h} n_{k} d s & \forall\left(\xi_{h}, \mu_{h}\right) \in\left(U_{h} \times M_{h}\right) .
\end{aligned}
$$

The seven equations (172)-(178) constitute a linear algebraic system for the seven scalar dependent variables in $\mathbf{U}_{h}, \wp_{h}$ and $\mathbf{P}_{h}$. The Dirichlet conditions on $\Gamma_{D}$ are included in the standard essential manner through the definitions (170)-(171). The spaces $U_{h}$ and $V_{h}$ are made of discontinuous functions over $\mathscr{T}_{h}$ and are used to approximate the primal and dual variables inside each element $K_{i}$. The spaces $M_{h, 0}^{u}$ and $M_{h, 0}^{p}$ are made of functions defined only at the nodes of $\mathscr{T}_{h}$ and are used to approximate the primal variables at each node. In particular, the function $\widehat{u}_{h}$ (resp., $\widehat{p}_{h}$ ) is the Lagrange multiplier that enforces the interelement continuity at $x=x_{k}, k=1, \ldots, \mathscr{K}_{h}-1$, of the normal component $\sigma_{h} n_{k}$ (resp., $v_{h} n_{k}$ ) and the Neumann boundary condition on $\Gamma_{N}$. In mechanical terms, $\widehat{u}_{h}$ and $\widehat{p}_{h}$ are referred to as interelement connectors (see [10] and the references cited therein).

\subsubsection{Static Condensation}

Looking at the structure of the discrete problem (172)-(178), one is tempted to conclude that there is a proliferation of unknowns which reflects into a very expensive (and complicated) numerical coding. However, all equations except (175) and (178) are completely local and, consequently, for each element $K_{k} \in \mathscr{T}_{h}$ the internal variables $\sigma_{h}$ and $u_{h}$, as well as $v_{h}$ and $p_{h}$, can be eliminated in favor of the nodal variables $\widehat{u}_{h}$ and $\widehat{p}_{h}$ and the problem data. This elimination procedure is referred to as static condensation and is the fundamental step that makes the hybridized method efficient and computationally competitive with standard displacement-based approaches. Static condensation can also be given an abstract form based on the concepts of local lifting and local solver which allows to interpret the elimination procedure as a (discrete) weak variational formulation of the original differential problem where the unknown is the hybrid variable. Such interpretation confers to the hybridization strategy a solid mathematical foundation and allows to apply standard functional analysis techniques to study well posedness and convergence of the hybridized finite element approximation (see [14] and [13] for a discussion in the case of second-order elliptic problems with diffusive and advective-diffusive operators). 
The application of static condensation to the problem at hand is far from trivial, since we deal with a non-scalar problem coupling fluid and solid equations. Details on the implementation are given below.

Consider a generic element $K_{k} \in \mathscr{T}_{h}$ and omit for notational brevity the suffix $K_{k}$ from all the involved quantities (whenever possible). Equation (172) can be written in matrix form as

$$
m_{u}^{-1} \mathbf{A} \sigma+\mathbf{B}^{T} \mathbf{u}-\mathbf{C}^{T} \widehat{\mathbf{u}}+m_{u}^{-1} m_{p} \mathbf{D} \wp+m_{u}^{-1} \mathbf{D} \mathbf{p}=\delta m_{u}^{-1} \frac{H_{V}}{\Delta t \lambda_{e}} \mathbf{D} \wp^{i}
$$

The quantities $\sigma$ and $\mathbf{u}$ are the column vectors (of dimension 2 and 1, respectively) containing the degrees of freedom for the restrictions $\left.\sigma_{h}\right|_{K_{k}}$ and $\left.u_{h}\right|_{K_{k}}$. The quantity $\widehat{\mathbf{u}}$ is the column vector (of dimension 2) containing the degrees of freedom of the restriction $\widehat{u}_{\partial K_{k}}$. The quantities $\wp$ and $\mathbf{p}$ are the column vectors (of dimension 1) containing the degrees of freedom of the restrictions $\left.\wp_{h}\right|_{K_{k}}$ and $\left.p_{h}\right|_{K_{k}}$, respectively. The quantities $m_{u}^{-1} \mathbf{A}, \mathbf{B}, \mathbf{C}$ and $\mathbf{D}$ are the matrices (of dimension $2 \times 2,1 \times 2,2 \times 2$ and $2 \times 1$, respectively) corresponding to the restrictions to the element $K_{k}$ of the bilinear forms $A, B, C$ and $D$, respectively.

Starting from the solid phase, we see that matrix $\mathbf{A}$ is invertible so that

$$
\sigma=m_{u} \mathbf{A}^{-1}\left[\mathbf{C}^{T} \widehat{\mathbf{u}}-\mathbf{B}^{T} \mathbf{u}\right]-\mathbf{A}^{-1} \mathbf{D}\left[m_{p} \wp+\mathbf{p}\right]+\mathbf{r}^{i}
$$

where $\mathbf{r}^{i}:=\delta \frac{H_{V}}{\Delta t \lambda_{e}} \mathbf{A}^{-1} \mathbf{D} \wp^{i}$. If $\lambda_{e}<+\infty$, we can eliminate $\wp_{h}$ in favor of $\widehat{u}_{h}$ in equation (173) to obtain

$$
\wp=-\lambda_{e} \mathbf{G} \widehat{\mathbf{u}}
$$

where $\mathbf{G}$ is the $1 \times 2$ matrix defined as $h_{k}^{-1}[-1+1]$ such that the matrix-vector product $\mathbf{G} \widehat{\mathbf{u}}$ is the constant approximation of $-\partial u / \partial x$ over the element $K_{k}$. Otherwise, if $\lambda_{e}=+\infty$, equation (173) becomes

$$
\int_{\partial K_{k}} \widehat{u}_{h} n_{k} d s=0 \quad \forall K_{k} \in \mathscr{T}_{h}
$$

which is the natural way to express material incompressibility in local weak form. As commonly done in biomechanical calculations, we assume $\lambda_{e}<+\infty$ (although close to the incompressibile limit) so that we use (180) into (179) to obtain

$$
\sigma=\mathscr{M} \widehat{\mathbf{u}}-m_{u} \mathbf{A}^{-1} \mathbf{B}^{T} \mathbf{u}-\mathbf{A}^{-1} \mathbf{D} \mathbf{p}+\mathbf{r}^{i},
$$

having set

Eq. (174) yields

$$
\mathscr{M}:=\mathbf{A}^{-1}\left(m_{u} \mathbf{C}+\lambda_{e} m_{p} \mathbf{D G}\right)
$$

$$
\mathbf{B} \sigma=-\mathbf{b}^{i+1},
$$

having set

$$
\mathbf{b}^{i+1}=h_{k} F^{i+1}\left(\bar{x}_{k}\right) .
$$

Moving to the fluid phase, Eq. (176) yields

$$
\mathbf{v}=-k^{(j)} \mathbf{A}^{-1}\left[\mathbf{C} \widehat{\mathbf{p}}-\mathbf{B}^{T} \mathbf{p}\right]
$$


where $k^{(j)}:=k_{\text {ref }} f\left(\phi_{0}-\left.\wp^{(j)}\right|_{K_{k}} / \lambda_{e}\right)$, while Eq. (177) yields

$$
\mathbf{B v}-\frac{h_{k}}{\lambda_{e} \Delta t} \wp=\mathbf{l}^{i+1}
$$

having set

$$
\mathbf{l}^{i+1}=h_{k} S^{i+1}\left(\bar{x}_{k}\right)-\frac{h_{k}}{\lambda_{e} \Delta t} \wp^{i} .
$$

Substituting (180) and (184) into (185) we are able to express the internal fluid pressure $\left.p_{h}\right|_{K_{k}}$ as a function of the sole hybrid variables $\left.\widehat{u}_{h}\right|_{\partial K_{k}}$ and $\left.\widehat{p}_{h}\right|_{\partial K_{k}}$ through the following relation

$$
k^{(j)} \mathbf{B} \mathbf{A}^{-1} \mathbf{B}^{T} \mathbf{p}=k^{(j)} \mathbf{B} \mathbf{A}^{-1} \mathbf{C} \widehat{\mathbf{p}}-\frac{h_{k}}{\Delta t} \mathbf{G} \widehat{\mathbf{u}}+\mathbf{l}^{i+1} .
$$

Because of Assumption 3.1 on the permeability $k$, the $1 \times 1$ matrix $\mathscr{B}_{p}:=k^{(j)} \mathbf{B A}^{-1} \mathbf{B}^{T}$ is symmetric and positive definite so that the above relation yields

$$
\mathbf{p}=\mathscr{Q} \widehat{\mathbf{p}}+\mathscr{R} \widehat{\mathbf{u}}+\mathscr{B}_{p}^{-1} \mathbf{l}^{i+1},
$$

having set

$$
\mathscr{Q}:=k^{(j)} \mathscr{B}_{p}^{-1} \mathbf{B} \mathbf{A}^{-1} \mathbf{C}, \quad \mathscr{R}:=-\frac{h_{k}}{\Delta t} \mathscr{B}_{p}^{-1} \mathbf{G} .
$$

We conclude the elimination procedure for the fluid phase by substituting (186) into (184) to obtain

$$
\begin{array}{r}
\mathbf{v}=\mathscr{L}_{p p} \widehat{\mathbf{p}}+\mathscr{L}_{p u} \widehat{\mathbf{u}}+\mathbf{b}_{p} \\
\mathscr{L}_{p p}=-k^{(j)} \mathbf{A}^{-1}\left[\mathbf{C}-\mathbf{B}^{T} \mathscr{Q}\right] \\
\mathscr{L}_{p u}=k^{(j)} \mathbf{A}^{-1} \mathbf{B}^{T} \mathscr{R} \\
\mathbf{b}_{p}=k^{(j)} \mathbf{A}^{-1} \mathbf{B}^{T} \mathscr{B}_{p}^{-1} \mathbf{l}^{i+1} .
\end{array}
$$

We proceed similarly for the solid phase by substituting (182) and (186) into (183) and obtain

$m_{u} \mathbf{B} \mathbf{A}^{-1} \mathbf{B}^{T} \mathbf{u}=\mathbf{B}\left[\mathscr{M}-\mathbf{A}^{-1} \mathbf{D} \mathscr{R}\right] \widehat{\mathbf{u}}-\mathbf{B} \mathbf{A}^{-1} \mathbf{D} \mathbf{Q} \widehat{\mathbf{p}}+\mathbf{B}\left[\mathbf{r}^{i+1}-\mathbf{A}^{-1} \mathbf{D} \mathscr{B}_{p}^{-1} \mathbf{l}^{i+1}\right]+\mathbf{b}^{i+1}$.

The $1 \times 1$ matrix $\mathscr{B}_{u}:=m_{u} \mathbf{B A}^{-1} \mathbf{B}^{T}$ is symmetric and positive definite so that the above relation yields

$$
\mathbf{u}=\widetilde{\mathscr{R}} \widehat{\mathbf{u}}+\widetilde{\mathscr{Q}} \widehat{\mathbf{p}}+\mathscr{B}_{u}^{-1} \mathbf{f}^{i+1},
$$

having set

$$
\widetilde{\mathscr{Q}}:=-\mathscr{B}_{u}^{-1} \mathbf{B} \mathbf{A}^{-1} \mathbf{D} \mathscr{Q} \quad \widetilde{\mathscr{R}}:=\mathscr{B}_{u}^{-1} \mathbf{B}\left[\mathscr{M}-\mathbf{A}^{-1} \mathbf{D} \mathscr{R}\right]
$$

and

$$
\mathbf{f}^{i+1}:=\mathbf{b}^{i+1}+\mathbf{B}\left[\mathbf{r}^{i}-\mathbf{A}^{-1} \mathbf{D} \mathscr{B}_{p}^{-1} \mathbf{l}^{i+1}\right]
$$


We conclude the elimination procedure for the solid phase by substituting (186) and (191) into (182) to obtain

$$
\begin{array}{r}
\sigma=\mathscr{L}_{u u} \widehat{\mathbf{u}}+\mathscr{L}_{u p} \widehat{\mathbf{p}}+\mathbf{b}_{u} \\
\mathscr{L}_{u u}=\mathscr{M}-\mathbf{A}^{-1}\left[m_{u} \mathbf{B}^{T} \widetilde{R}+\mathbf{D} \mathscr{R}\right] \\
\mathscr{L}_{u p}=-\mathbf{A}^{-1}\left[m_{u} \mathbf{B}^{T} \widetilde{\mathscr{Q}}+\mathbf{D} \mathscr{Q}\right] \\
\mathbf{b}_{u}=\mathbf{r}^{i}-m_{u} \mathbf{A}^{-1} \mathbf{B}^{T} \mathscr{B}_{u}^{-1} \mathbf{f}^{i+1}-\mathbf{A}^{-1} \mathbf{D} \mathscr{B}_{p}^{-1} \mathbf{l}^{i+1} .
\end{array}
$$

The above illustrated static condensation procedure corresponds to Gaussian elimination, at the level of the element $K_{k} \in \mathscr{T}_{h}$, of the internal variables $\left.\sigma_{h}\right|_{K_{k}},\left.u_{h}\right|_{K_{k}}$ and $\left.v_{h}\right|_{K_{k}},\left.p_{h}\right|_{K_{k}}$ in favor of $\left.\widehat{u}_{h}\right|_{\partial K_{k}}$ and $\left.\widehat{p}_{h}\right|_{\partial K_{k}}$ (see [2]). Over the last years, the use of static condensation has been extended also to the class of Discontinuous Galerkin (DG) methods, giving rise to the so-called Hybridized DG finite element formulation. A complete overview and analysis of HDG methods applied to the solution of an elliptic model problem in multiple spatial dimensions can be found in [15].

\subsection{The Linear Algebraic System}

Having eliminated all the internal variables, it only remains to enforce the continuity of interelement normal stress and normal Darcy's velocity and enforce the Neumann boundary conditions, see (175) and (178). Without loss of generality, we show these steps in the case where $\Gamma_{D, p}=\left\{x_{\text {start }}\right\}, \Gamma_{N}=\left\{x_{\text {end }}\right\}$ and $\Gamma_{D, v}=\emptyset$. For each element $K_{k} \in \mathscr{T}_{h}, k=1, \ldots, \mathscr{K}_{h}$, and for any function $\eta_{h} \in \mathbb{P}_{1}\left(K_{k}\right)$ we set $\eta_{h}(x)=\eta^{1} \tau_{1}(x)+\eta^{2} \tau_{2}(x)$ where $\eta^{1}, \eta^{2}$ are the degrees of freedom of $\eta_{h}$ and $\tau_{1}, \tau_{2}$ are the two (local) Lagrange basis functions ("hat functions") associated with nodes $x_{k-1}$ and $x_{k}$, respectively. Conditions (175) and (178) give rise to the following $2 M_{h}$ equations:

$$
\begin{array}{rlrl}
\sigma_{K_{k-1}}^{2}\left(\widehat{u}_{k-1}, \widehat{u}_{k}, \widehat{p}_{k-1}, \widehat{p}_{k}\right) & =\sigma_{K_{k}}^{1}\left(\widehat{u}_{k}, \widehat{u}_{k+1}, \widehat{p}_{k}, \widehat{p}_{k+1}\right) & k & =1, \ldots, \mathscr{K}_{h}-1(1 \\
\sigma_{K_{k}}^{2}\left(\widehat{u}_{k-1}, \widehat{u}_{k}, \widehat{p}_{k-1}, \widehat{p}_{k}\right) & =g^{i+1} & k & =\mathscr{K}_{h}, \\
v_{K_{k-1}}^{2}\left(\widehat{u}_{k-1}, \widehat{u}_{k}, \widehat{p}_{k-1}, \widehat{p}_{k}\right) & =v_{K_{k}}^{1}\left(\widehat{u}_{k}, \widehat{u}_{k+1}, \widehat{p}_{k}, \widehat{p}_{k+1}\right) & k & =1, \ldots, \mathscr{K}_{h}-1 \\
v_{K_{k}}^{2}\left(\widehat{u}_{k-1}, \widehat{u}_{k}, \widehat{p}_{k-1}, \widehat{p}_{k}\right) & =0 & k & =\mathscr{K}_{h} .
\end{array}
$$

Looking at the above relations, we see that conditions (175) and (178) are nonlocal because, for each $k=1, \ldots, \mathscr{K}_{h}-1$, they couple the degrees of freedom $\widehat{u}_{k-1}, \widehat{u}_{k}, \widehat{u}_{k+1}$ with $\widehat{p}_{k-1}, \widehat{p}_{k}, \widehat{p}_{k+1}$, giving rise to the following linear algebraic block system

$$
\left[\begin{array}{ll}
\mathbf{M}_{u u} & \mathbf{M}_{u p} \\
\mathbf{M}_{p u} & \mathbf{M}_{p p}
\end{array}\right]\left[\begin{array}{l}
\widehat{\mathbf{u}} \\
\widehat{\mathbf{p}}
\end{array}\right]=\left[\begin{array}{l}
\mathbf{b}_{u} \\
\mathbf{b}_{p}
\end{array}\right]
$$

in which $\mathbf{M}_{u u}, \mathbf{M}_{u p}, \mathbf{M}_{p u}$ and $\mathbf{M}_{p p}$ are tridiagonal square matrices of size equal to $\mathscr{K}_{h}, \widehat{\mathbf{u}}$ and $\widehat{\mathbf{p}}$ are the vectors of nodal unknowns $\widehat{u}_{k}=\widehat{u}_{h}\left(x_{k}\right)$ and $\widehat{p}_{k}=\widehat{p}_{h}\left(x_{k}\right)$, both of size $\mathscr{K}_{h}$, and $\mathbf{b}_{u}, \mathbf{b}_{p}$ are the load vectors, both of size $\mathscr{K}_{h}$. 
Some comments about the solution of the linear system (200) are in order. First, we notice that matrix $\mathbf{M}_{u u}$ is symmetric and positive definite, whereas $\mathbf{M}_{p p}$ is symmetric and negative definite. These properties ensure that system (200) is uniquely solvable in the stationary case (equivalent to setting $1 / \Delta t=0$ ). Second, to prove that system (200) is uniquely solvable also in the time-dependent case we can follow the same arguments based on the saddle-point theory as in [21], Chapter 3. Third, to enforce the homogeneous Dirichlet boundary on $\Gamma_{D, p}$ we do not eliminate the rows and columns associated with the respective unknowns $\left(\widehat{u}_{h}\left(x_{\text {start }}\right)\right.$ and $\left.\widehat{p}_{h}\left(x_{\text {start }}\right)\right)$, rather, we set (using Matlab notation):

$$
\begin{array}{cccc}
\mathbf{M}_{u u}(1,:)=0, & \mathbf{M}_{u u}(1,1)=1, & \mathbf{M}_{u p}(1,:)=0, & \mathbf{b}_{u}(1)=0 \\
\mathbf{M}_{p u}(1,:)=0, & \mathbf{M}_{p p}(1,:)=0, & \mathbf{M}_{p p}(1,1)=1, & \mathbf{b}_{p}(1)=0 .
\end{array}
$$

This simplifies considerably the coding and can be extended in a straightforward manner to multi-dimensions. Since we adopt a direct solver in numerical computations (the \command in Matlab) the use of (201)-(202) amounts to enforcing exactly the boundary conditions $u\left(x_{\text {start }}, t\right)=p\left(x_{\text {start }}, t\right)=0$ for all $t \in\left(t_{\text {start }}, t_{\text {end }}\right)$.

\subsection{Validation of the numerical method}

The validity of the DMH method described in the previous sections is assessed by means of four test cases (denoted by $\mathrm{V}_{1}, \mathrm{~V}_{2}, \mathrm{~V}_{3}$ and $\mathrm{V}_{4}$, defined in Sections 4.5.1-4.5.4), where numerical and analytical solutions are compared for various spatial and temporal discretizations. In the following, we will consider uniform spatial and temporal grid size parameters defined as $h=L / \mathscr{K}_{h}$ and $\Delta t=T / r$, respectively. The accuracy of the approximation provided by the hybrid variables $\widehat{u}_{h}$ and $\widehat{p}_{h}$ is measured by computing the errors $u-u_{h}^{*}$ and $p-p_{h}^{*}$, where $u_{h}^{*}$ and $p_{h}^{*}$ are the conforming $\mathbb{P}_{1}$-interpolants of the nodal values $\widehat{u}_{k}$ and $\widehat{p}_{k}, k=0, \ldots, \mathscr{K}_{h}$, computed by solving the DMH linear algebraic system (200). Standard error estimates valid for 2 nd order elliptic problems predict an optimal convergence rate of $\mathscr{O}\left(h^{2}\right)$ in the $L^{2}$ norm for $u_{h}^{*}$ and $p_{h}^{*}$ and for $\sigma_{h}$ and $v_{h}$, whereas the expected convergence rate for $u_{h}$ and $p_{h}$ is only $\mathscr{O}(h)$ in the $L^{2}$ norm (for all the theoretical details, see $[2,4,48])$. In the following, for any function $w=w(x, t)$, we consider the norms

$$
\|w\|_{Q}:=\sup _{t \in\left[t_{\text {start }}, t_{\text {end }}\right]}\|w(t)\|_{L^{2}(\Omega)}, \quad\|w\|_{\infty}:=\sup _{t \in\left[t_{\text {start }}, t_{\text {end }}\right]}\|w(t)\|_{\infty} .
$$

If $w$ does not depend on time, we simply have

$$
\|w\|_{Q}=\left(\int_{x_{\text {start }}}^{x_{\text {end }}} w^{2}(x) d x\right)^{1 / 2}, \quad\|w\|_{\infty}=\sup _{x \in\left(x_{\text {start }}, x_{\text {end }}\right)}|w(x)| .
$$

Even though the poro-elastic and poro-visco-elastic models considered in this article go beyond the elliptic theory, it is still very interesting to compare the results we obtain with those available in simpler cases. To facilitate this comparison, we consider four test cases of increasing complexity, as summarized in Table 1 and detailed in the following sub-sections. 


\begin{tabular}{|c|c|c|}
\hline Test Case & Permeability & Data \\
\hline $\mathrm{V}_{1}$ & constant & constant in space and time \\
$\mathrm{V}_{2}$ & constant & varying in space and time \\
$\mathrm{V}_{3}$ & varying as in (7) & varying in space, constant in time \\
$\mathrm{V}_{4}$ & varying as in (7) & varying in space and time \\
\hline
\end{tabular}

Table 1 Summary of the main features of the four test cases used for the numerical validation of the DMH method.

\subsubsection{Validation test case $V_{1}$}

Let us consider problem (138)-(143) with $\delta=0$ in the domain $\Omega=(0,1)$, so that $L=1 \mathrm{~cm}$, with the boundary conditions $u=p=0$ at $x_{\text {start }}=0$, and $\sigma n=g_{1}$ and $v n=\psi_{1}$ at $x_{\text {end }}=1$. We assume volumetric and boundary source terms to be constant and given by

$$
F_{1}=0.3 \text { dyne } \mathrm{cm}^{-3}, \quad S_{1}=0.3 \mathrm{~s}^{-1}, \quad g_{1}=-0.3 \text { dyne }^{-2}, \quad \psi_{1}=-3 \mathrm{cms}^{-1} .
$$

We also assume that porosity and permeability are constant and given by $\phi=\phi_{0}=$ 0.5 and $k=k_{r e f}=1 \mathrm{~cm}^{3} \mathrm{sg}^{-1}$, respectively. In this case, the problem admits the exact solution:

$$
\begin{gathered}
u(x)=\frac{x}{H_{A}}\left[F_{1}\left(L-\frac{x}{2}\right)+g_{1}\right]-\frac{x^{2}}{2 H_{A} k_{r e f}}\left[\psi_{1}-S_{1}\left(L-\frac{x}{3}\right)\right], \\
p(x)=\frac{x}{k_{r e f}}\left[S_{1}\left(L-\frac{x}{2}\right)-\psi_{1}\right], \quad \wp(x)=-\frac{\lambda_{e}}{H_{A}}(\sigma(x)+p(x)), \\
\sigma(x)=g_{1}+F_{1}(L-x), \quad v(x)=\psi_{1}+S_{1}(x-L),
\end{gathered}
$$

where $H_{A}=\lambda_{e}+2 \mu_{e}$ is the aggregate elastic modulus, with $\lambda_{e}=\mu_{e}=1 \mathrm{dyne} \mathrm{cm}^{-2}$. Since the exact solution is stationary, we solve directly the stationary problem by setting $1 / \Delta t=0$. We consider decreasing grid sizes $h=L / \mathscr{K}_{h}$, with $\mathscr{K}_{h}=$ $[5,10,20,40,80,160,320]^{T}$.

Numerical results (not reported here) show that $\sigma_{h}$ and $v_{h}$ are exact up to machine precision and system conditioning. This accuracy is mathematically to be ascribed to the fact that both $\sigma$ and $v$ belong to the finite element space (168). Mechanically, it expresses the evidence that the DMH scheme satisfies the linear stress patch test (see [27] and [56] for a discussion of this important issue). The optimal accuracy of the hybrid variables is demonstrated in Fig. 1. Fig. 2 shows the behavior of the approximation of the elastic pressure parameter. Linear convergence is achieved in the $L^{2}$ norm, consistently with the fact that we are using a locally constant approximation of $\wp$ whereas second-order accuracy is obtained in the $L^{\infty}$ norm. This result is consistent with theoretical conclusions valid in the elliptic case where superconvergence of the internal variables is achieved at the center of mass of each element $K_{k}$ (cf. [2] and [4] for the proof).

\subsubsection{Validation test case V2}

Let us now consider problem (138)-(143) with $\delta=1$ in the domain $\Omega=(0,1)$, so that $L=1 \mathrm{~cm}$, with the boundary conditions $u=p=0$ at $x_{\text {start }}=0$, and $\sigma n=$ 


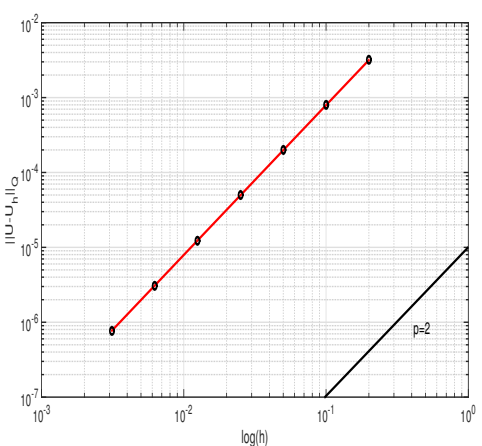

(a) $\left\|u-u_{h}^{*}\right\|_{Q}$

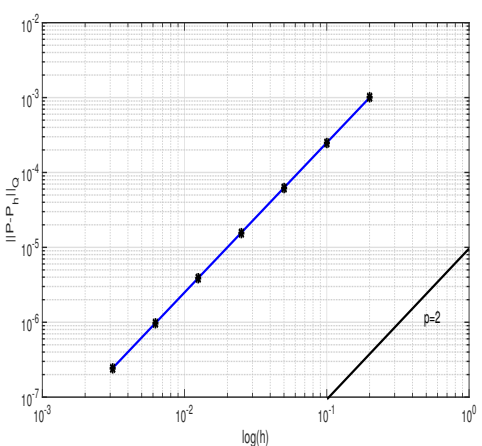

(b) $\left\|p-p_{h}^{*}\right\|_{Q}$

Fig. 1 Validation test $\mathrm{V}_{1}$. Discretization errors for the hybrid variables. The convergence rate is optimal and equal to $\mathscr{O}\left(h^{2}\right)$ with respect to $h$.

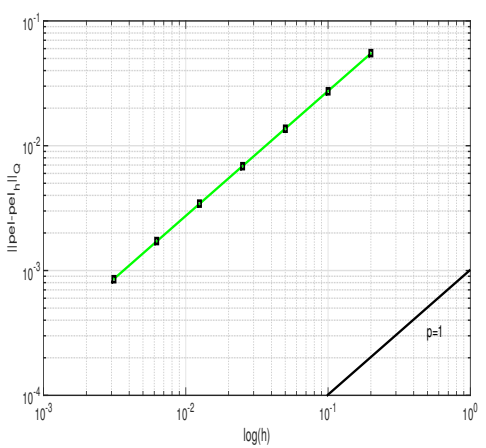

(a) $\left\|\wp-\wp_{h}\right\|_{Q}$

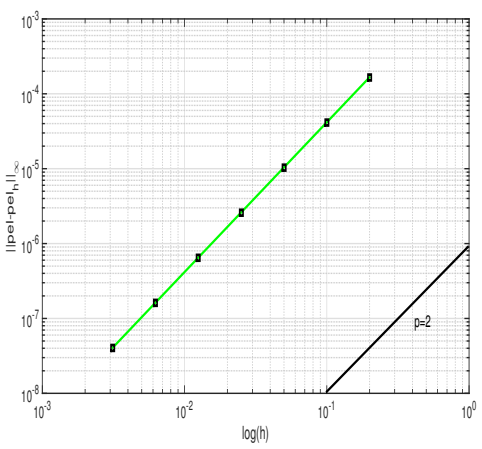

(b) $\left\|\wp-\wp_{h}\right\|_{\infty}$

Fig. 2 Validation test $V_{1}$. Approximation of the elastic pressure. Superconvergence $\left(\mathscr{O}\left(h^{2}\right)\right)$ is obtained at mesh midpoints.

$g_{2}(t)$ and $v n=\psi_{2}(t)$ at $x_{\text {end }}=1$. Now volumetric and boundary source terms are not constant, neither in space nor in time. Thus, considering the time interval $\left(t_{\text {start }}, t_{\text {end }}\right)=(0, T)$, with $T=0.1 \mathrm{~s}$, and the spatial and temporal shape functions $\chi(x)=\sin \left(\omega_{x} x\right)$ and $\tau(t)=\sin ^{2}\left(\omega_{t} t\right)$, with $\omega_{x}=8 / L$ and $\omega_{t}=8 / T$, we assume that the data are given by:

$$
\begin{aligned}
F_{2}(x, t) & =-\left\{U_{r e f} \chi^{\prime \prime}(x)\left[H_{A} \tau(t)+\delta H_{V} \tau^{\prime}(t)\right]-P_{r e f} \tau(t) \chi^{\prime}(x)\right\}, \\
S_{2}(x, t) & =U_{r e f} \tau^{\prime}(t) \chi^{\prime}(x)-k_{r e f} P_{r e f} \chi^{\prime \prime}(x) \tau(t), \\
g_{2}(t) & =U_{r e f} \chi^{\prime}(x)\left[H_{A} \tau(t)+\delta H_{V} \tau^{\prime}(t)\right]-P_{r e f} \tau(t) \chi(L), \\
\psi_{2}(t) & =-k_{r e f} P_{r e f} \tau(t) \chi^{\prime}(L),
\end{aligned}
$$

with $U_{r e f}=0.1 \mathrm{~cm}, P_{r e f}=0.3 \mathrm{dyne}^{-2}, H_{A}=\lambda_{e}+2 \mu_{e}=3 \mathrm{dyne} \mathrm{cm}^{-2}, \lambda_{e}=$ $\mu_{e}=1$ dyne $\mathrm{cm}^{-2}$ and $H_{V}=\lambda_{v}+2 \mu_{v}=0.5774$ dyne $\mathrm{cm}^{-2}$. As in test case $\mathrm{V}_{1}$, we assume that porosity and permeability are constant and given by $\phi=\phi_{0}=0.5$ 
and $k=k_{r e f}=1 \mathrm{~cm}^{3} \mathrm{sg}^{-1}$, respectively. In this case the problem admits the exact solution:

$$
\begin{aligned}
u(x, t) & =U_{r e f} \chi(x) \tau(t), \\
p(x, t) & =P_{r e f} \chi(x) \tau(t), \\
\sigma(x, t) & =U_{r e f} \chi^{\prime}(x)\left[H_{A} \tau(t)+\delta H_{V} \tau^{\prime}(t)\right]-P_{r e f} \chi(x) \tau(t), \\
v(x, t) & =-k_{r e f} P_{r e f} \chi^{\prime}(x) \tau(t), \\
\wp(x, t) & =-\lambda_{e} U_{r e f} \chi^{\prime}(x) \tau(t),
\end{aligned}
$$

which now depends on both space and time. We compute the numerical approximation of the solution considering uniform spatial and temporal grid size parameters defined as $h=L / \mathscr{K}_{h}$ and $\Delta t=T / r$, with $\mathscr{K}_{h}=[5,10,20,40,80,160,320]^{T}$ and $r=[5,10,20,40,80,160,320]^{T}$.

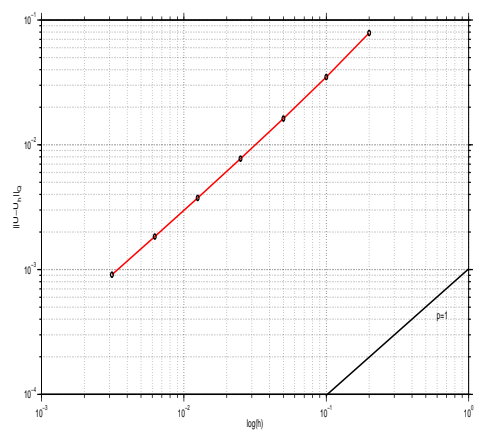

(a) $\left\|u-u_{h}^{*}\right\|_{Q}$

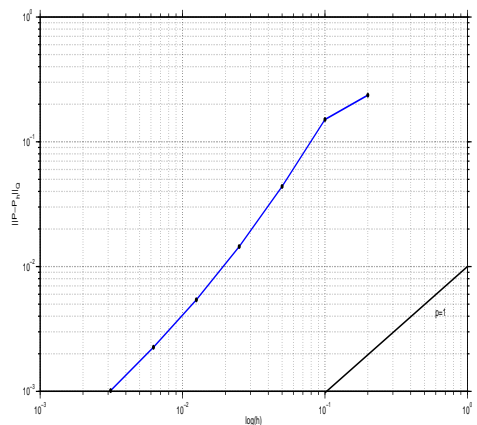

(b) $\left\|p-p_{h}^{*}\right\|_{Q}$

Fig. 3 Validation test $V_{2}$. Discretization errors for the hybrid variables. The convergence rate is sub-optimal for both variables and equal to $\mathscr{O}(h)$ with respect to $h$.

In this case, as illustrated in Figures 3-5, all the approximate variables converge to the corresponding exact ones with linear rate with respect to $h$, except the variable $\sigma_{h}$ (approximate total stress) which continues to converge with an optimal rate $\left(\mathscr{O}\left(h^{2}\right)\right)$. The degradation of the convergence order of the DMH method is to be ascribed to the choice of the Backward Euler method as time-advancing scheme which is well-known to be only first-order accurate in time [44]. It is remarkable to notice that the stress variable is not affected by such a degradation, since a time derivative is present in the constitutive equation for the stress but not in the equation for the balance of linear momentum (138). This is not the case for the discharge velocity because the time derivative of the fluid content $\zeta$ appears directly in the fluid mass balance equation (139).

\subsubsection{Validation test case $V_{3}$}

Let us consider again problem (138)-(143) with $\delta=0$ in the domain $\Omega=(-1,1)$, so that $L=2 \mathrm{~cm}$, with the boundary conditions $u=p=0$ at $x_{\text {start }}=-1$, and 


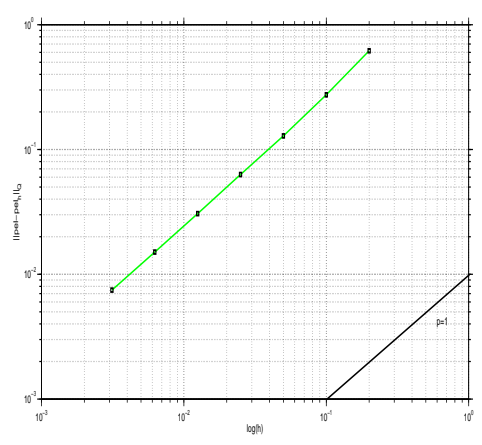

(a) $\left\|\wp-\wp \varkappa_{h}\right\|_{Q}$

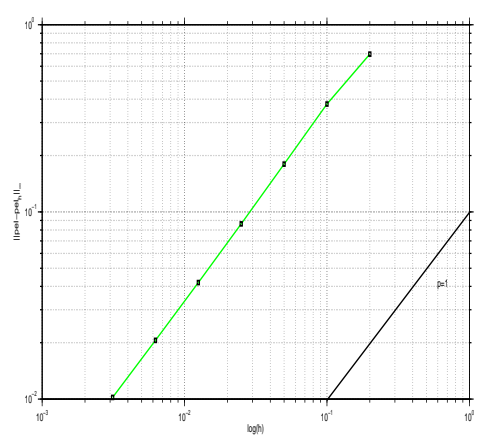

(b) $\|\wp-\wp\|_{h}$

Fig. 4 Validation test $V_{2}$. Approximation of the elastic pressure.

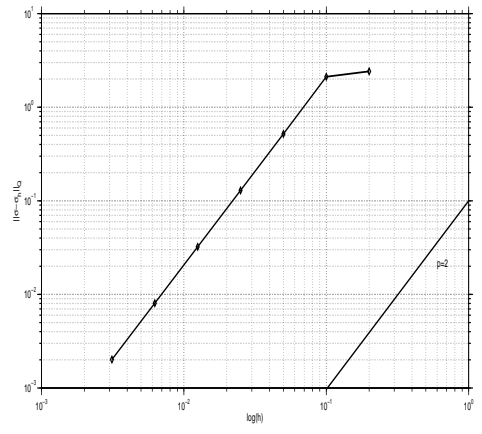

(a) $\left\|\sigma-\sigma_{h}\right\|_{Q}$

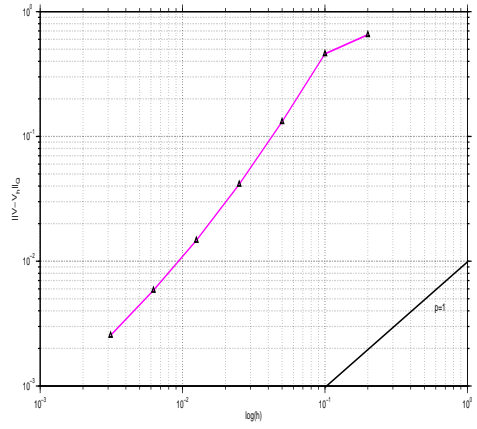

(b) $\left\|v-v_{h}\right\|_{Q}$

Fig. 5 Validation test $\mathrm{V}_{2}$. Discretization errors for the total stress and Darcy's velocity. The convergence rate is optimal for the stress $\left(\mathscr{O}\left(h^{2}\right)\right)$ but is sub-optimal for the velocity $(\mathscr{O}(h))$.

$\sigma n=g_{3}$ and $v n=\psi_{3}$ at $x_{\text {end }}=1$. Volumetric and boundary source terms are given by:

$$
\begin{aligned}
F_{3}(x) & =-\left[U_{r e f} H_{A} \chi^{\prime \prime}(x)-P_{r e f} \chi^{\prime}(x)\right], \\
S_{3}(x) & =-k_{r e f} P_{r e f} \chi^{\prime \prime}(x) \Theta(x)-k_{r e f} P_{r e f} U_{r e f} \chi^{\prime}(x) \chi^{\prime \prime}(x) \Xi(x), \\
g_{3} & =U_{r e f} H_{A} \chi\left(x_{\text {end }}\right)-P_{r e f} \chi\left(x_{\text {end }}\right), \\
\psi_{3} & =-k_{\text {ref }} P_{r e f} \chi^{\prime}\left(x_{\text {end }}\right) \Theta\left(x_{\text {end }}\right),
\end{aligned}
$$

where:

$$
\begin{gathered}
\chi(x)=\sin \left(\omega_{x} x\right), \quad \Phi(x)=\phi_{0}+U_{\text {ref }} \chi^{\prime}(x), \\
\Theta(x)=\frac{\Phi^{3}(x)}{[1-\Phi(x)]^{2}}, \quad \Xi(x)=\frac{\Phi^{2}(x)[3-\Phi(x)]}{[1-\Phi(x)]^{3}},
\end{gathered}
$$

with $\omega_{x}=2 \pi / L, U_{r e f}=0.1 \mathrm{~cm}, P_{r e f}=1$ dyne $\mathrm{cm}^{-2}, H_{A}=3$ dyne $\mathrm{cm}^{-2}$ and $\phi_{0}=$ 0.5 . Unlike previous test cases, the porosity $\phi$ is now allowed to vary with the 
derivative of the displacement within the range $\left[\Phi_{\min }, \Phi_{\max }\right]$, where $0<\Phi_{\min }<$ $\Phi_{\max }<1$, in such a way that the permeability $k$, expressed by the nonlinear relation (7), satisfies

$$
0<k_{\text {ref }} \frac{\Phi_{\min }^{3}}{\left(1-\Phi_{\min }\right)^{2}} \leq k(\phi) \leq k_{\text {ref }} \frac{\Phi_{\max }^{3}}{\left(1-\Phi_{\max }\right)^{2}} .
$$

The above limitations on porosity and permeability are the same as those adopted in [8]. Here we set $\Phi_{\text {min }}=0.125, \Phi_{\text {max }}=0.875$ and $k_{r e f}=1 \mathrm{~cm}^{3} \mathrm{sg}^{-1}$. In this case the problem admits the exact solution:

$$
\begin{aligned}
u(x) & =U_{r e f} \chi(x), \\
p(x) & =P_{r e f} \chi(x), \\
\sigma(x) & =U_{r e f} H_{A} \chi^{\prime}(x)-P_{r e f} \chi(x), \\
v(x) & =-k_{r e f} P_{r e f} \chi^{\prime}(x) \Theta(x), \\
\wp(x) & =-\lambda_{e} U_{r e f} \chi^{\prime}(x) .
\end{aligned}
$$

Since the problem is stationary, we compute directly the stationary solution by setting $1 / \Delta t=0$ in the numerical code. The Picard iteration (158)-(157) is terminated at the first value of $j$, say $j^{*}$, such that the maximum relative increment defined as $\left\|X^{\left(j^{*}\right)}-X^{\left(j^{*}-1\right)}\right\| /\left\|X^{\left(j^{*}\right)}\right\|$ is less than a prescribed tolerance $\varepsilon$, where $\varepsilon=10^{-3}$ and $X$ is any variable in the set $\left\{u_{h}, p_{h}, \widehat{u}_{h}^{*}, \widehat{p}_{h}^{*}, \sigma_{h}, v_{h}\right\}$. We consider decreasing

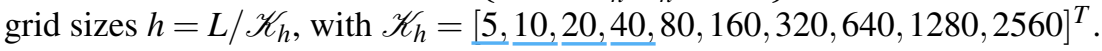

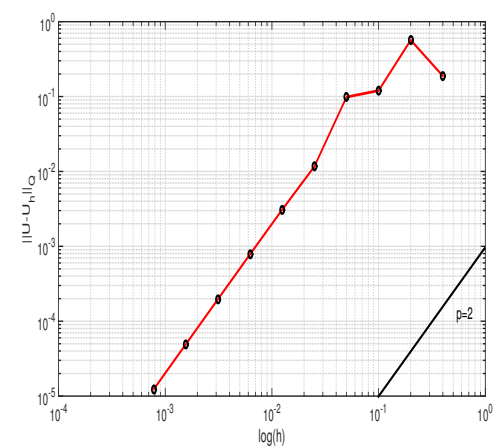

(a) $\left\|u-u_{h}^{*}\right\|_{Q}$

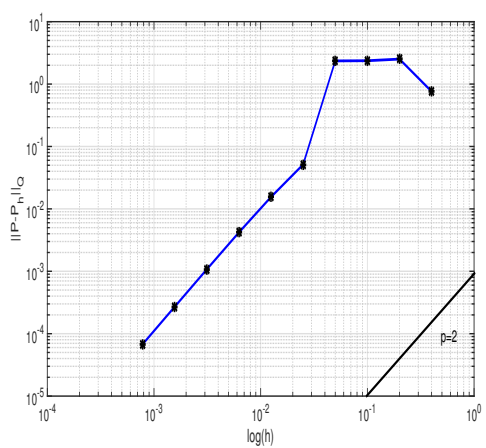

(b) $\left\|p-p_{h}^{*}\right\|_{Q}$

Fig. 6 Validation test $\mathrm{V}_{3}$. Discretization errors for the hybrid variables show that the convergence rate $\left(\mathscr{O}\left(h^{2}\right)\right)$ is optimal for both variables.

The simulation results are reported in figures 6-8. Interestingly, even in this fully nonlinear case where porosity and permeability vary with the problem solution, the asymptotic convergence rates for the various approximation errors are the same optimal values as those in the basic linear test case $V_{1}$ with constant coefficients. This example demonstrates the ability of the proposed DMH method to provide a reliable approximation of the nonlinear poro-elastic problem at hand. 


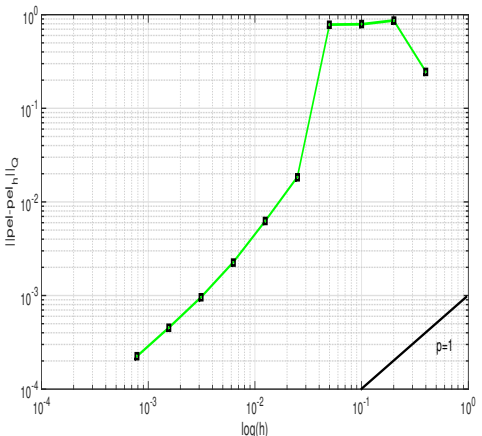

(a) $\left\|\wp-\wp_{h}\right\|_{Q}$

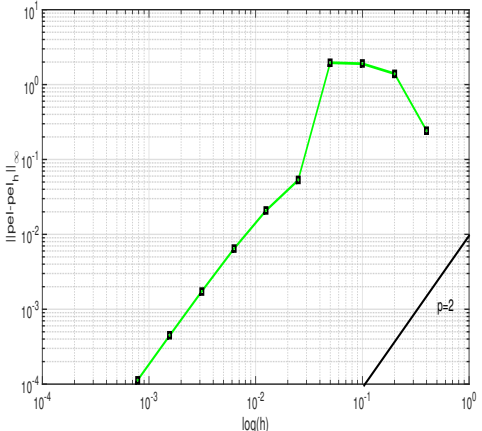

(b) $\left\|\wp-\wp_{h}\right\|_{\infty}$

Fig. 7 Validation test $\mathrm{V}_{3}$. Approximation of the elastic pressure show that superconvergence $\left(\mathscr{O}\left(h^{2}\right)\right)$ is obtained at mesh midpoints.

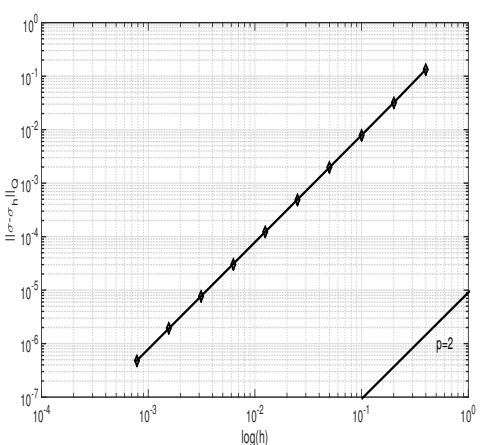

(a) $\left\|\sigma-\sigma_{h}\right\|_{Q}$

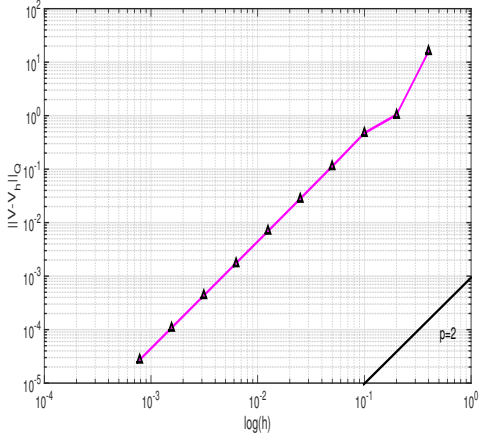

(b) $\left\|v-v_{h}\right\|_{Q}$

Fig. 8 Validation test $V_{3}$. Discretization errors for the total stress and Darcy's velocity show that the convergence rate $\left(\mathscr{O}\left(h^{2}\right)\right)$ is optimal for both variables.

\subsubsection{Validation test case $V_{4}$}

This test case is the time dependent version of the previous test case $\mathrm{V}_{3}$. Let us consider again problem (138)-(143) with $\delta=1$ in the space-time domain $(-1,1) \times$ $(0, T)$, so that $L=2 \mathrm{~cm}$ and $T=2 \mathrm{~s}$, with the boundary conditions $u=p=0$ at $x_{\text {start }}=-1$, and $\sigma n=g_{4}(t)$ and $v n=\psi_{4}(t)$ at $x_{\text {end }}=1$. Porosity and permeability are nonlinear functions of the solution, as described in test case $\mathrm{V}_{3}$. Now the volumetric and boundary source terms are time-dependent and are given by:

$$
\begin{aligned}
F_{4}(x, t) & =-\left[U_{r e f} \chi^{\prime \prime}(x)\left(H_{A} \tau(t)+\delta H_{V} \tau^{\prime}(t)\right)-P_{r e f} \tau(t) \chi^{\prime}(x)\right], \\
S_{4}(x, t) & =U_{r e f} \chi^{\prime}(x) \tau^{\prime}(t)-P_{r e f} k_{r e f} \chi^{\prime \prime}(x) \tau(t) \Theta(x, t)-k_{r e f} P_{r e f} U_{r e f} \chi^{\prime}(x) \chi^{\prime \prime}(x) \tau^{2}(t) \Xi(x, t), \\
g_{4}(t) & =U_{r e f} \chi^{\prime}\left(x_{\text {end }}\right)\left(H_{A} \tau(t)+\delta H_{V} \tau^{\prime}(t)\right)-P_{r e f} \tau(t) \chi\left(x_{\text {end }}\right), \\
\psi_{4}(t) & =-k_{r e f} P_{r e f} \Theta\left(x_{\text {end }}\right) \chi^{\prime}\left(x_{\text {end }}\right) \tau(t),
\end{aligned}
$$


where:

$$
\begin{gathered}
\chi(x)=\sin \left(\omega_{x} x\right), \quad \tau(t)=\sin \left(\omega_{t} t\right), \quad \Phi(x, t)=\phi_{0}+U_{\text {ref }} \chi^{\prime}(x) \tau(t), \\
\Theta(x, t)=\frac{\Phi^{3}(x, t)}{[1-\Phi(x, t)]^{2}}, \quad \Xi(x, t)=\frac{\Phi^{2}(x, t)[3-\Phi(x, t)]}{[1-\Phi(x, t)]^{3}},
\end{gathered}
$$

with $\omega_{t}=2 \pi / T$ and all the other parameter values given as in test case $\mathrm{V}_{3}$. In this case the problem admits the exact solution:

$$
\begin{aligned}
u(x, t) & =U_{\text {ref }} \chi(x) \tau(t), \\
p(x, t) & =P_{r e f} \chi(x) \tau(t), \\
\sigma(x, t) & =U_{r e f} \chi^{\prime}(x)\left(H_{A} \tau(t)+\delta H_{V} \tau^{\prime}(t)\right)-P_{r e f} \chi(x) \tau(t), \\
v(x, t) & =-P_{r e f} k_{r e f} \Theta(x, t) \chi^{\prime}(x) \tau(t), \\
\wp(x, t) & =-\lambda_{e} U_{r e f} \chi^{\prime}(x) \tau(t) .
\end{aligned}
$$

We compute the numerical approximation of the solution considering uniform spatial and temporal grid size parameters defined as $h=L / \mathscr{K}_{h}$ and $\Delta t=T / r$, with $\mathscr{K}_{h}=[5,10,20,40,80,160,320,640,1280,2560]^{T}$ and $r=[5,10,20,40$, $80,160,320,640,1280,2560]^{T}$.

The simulation results are reported in the set of figures 9-12. This corresponds to numerically solving the fully nonlinear coupled poro-visco-elastic system in the case where the permeability is described by the Carman-Kozeny relation (7). We see that the first-order temporal accuracy of the BE method spoils the superconvergence property of the DMH that was achieved in the stationary test case $\mathrm{V}_{3}$. In particular, the convergence of the hybrid variables reduces from a quadratic (Fig. 6) to a linear rate (Fig. 9). No superconvergence at the mesh centers of mass is obtained in the time dependent case (Fig. 10(b)) unlike the stationary case (Fig. 7(b)). The Darcy velocity field suffers a similar reduction in the convergence rate which lowers from second order (Fig. 8(b)) to linear order (Fig. 12(a)). It is remarkable to notice that the decrease in accuracy for the Darcy velocity occurs in correspondance of small values of the discretization parameters, since for larger values of $h$ and $\Delta t$ the slope of the error curve is close to the optimal value $p=2$ (Fig. 12(b)). This seems to indicate that problem nonlinearity drives a smooth transition of the accuracy behaviour of the method as a function of the spatial and temporal discretization parameters, in such a manner that the degradation effect due to the $\mathrm{BE}$ method can be actually experienced only in the limit of very small mesh parameter size. The accuracy of the approximation of the total stress is the sole to be preserved from stationary to time dependent conditions (compare Fig. 8(a) with Fig. 11). The reason for this exception is the same as that pointed out for test case $\mathrm{V}_{2}$.

4.6 Numerical study of the influence of data regularity on the energy estimates

We are now in a position to use the DMH method to investigate numerically the energy estimates obtained in Section 3.6. The 1D counterparts of the 3D expres- 


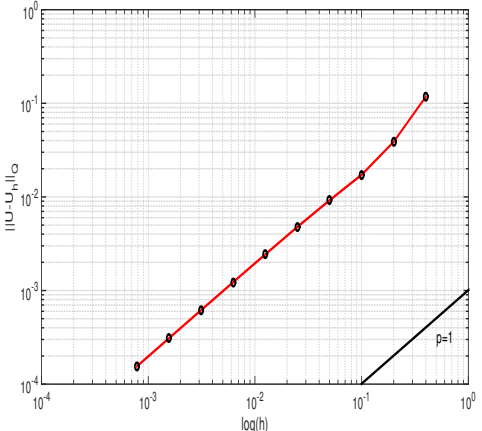

(a) $\left\|u-u_{h}^{*}\right\|_{Q}$

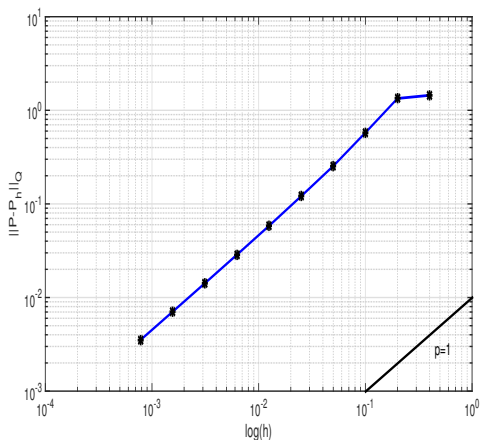

(b) $\left\|p-p_{h}^{*}\right\|_{Q}$

Fig. 9 Validation test $\mathrm{V}_{4}$. Discretization errors for the hybrid variables show that the convergence rate $(\mathscr{O}(h))$ is sub-optimal for both variables.

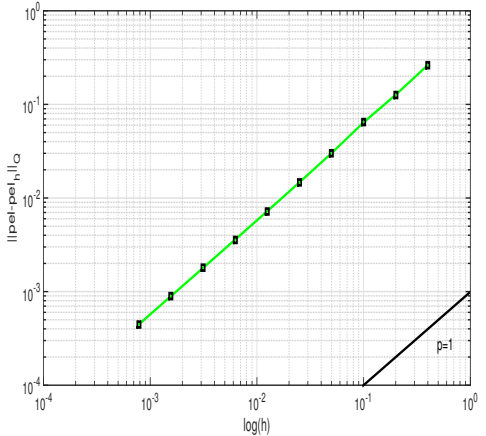

(a) $\left\|\wp-\wp_{h}\right\|_{Q}$

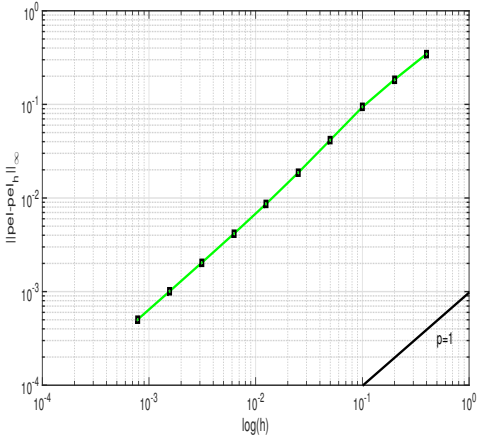

(b) $\left\|\wp-\wp_{h}\right\|_{\infty}$

Fig. 10 Validation test $\mathrm{V}_{4}$. Approximation of the elastic pressure shows that the convergence rate is $\mathscr{O}(h)$ in both norms, so that no superconvergence is obtained at the mesh centers of mass.

sions for the energies given in (126), (127) and (128) are:

$$
\begin{array}{rlrl}
E_{e}(u(t)) & =\frac{1}{2}\left(\lambda_{e}+4 \mu_{e}\right)\left\|\frac{\partial u(x, t)}{\partial x}\right\|_{0}^{2} & & \forall t \in\left(t_{\text {start }}, t_{\text {end }}\right), \\
E_{v}(u(t))=\frac{1}{2}\left(\lambda_{v}+4 \mu_{v}\right)\left\|\frac{\partial u(x, t)}{\partial x}\right\|_{0}^{2} & \forall t \in\left(t_{\text {start }}, t_{\text {end }}\right), \\
E_{p}(p(t))=\left\|\sqrt{k} \frac{\partial p(x, t)}{\partial x}\right\|_{0}^{2} & \forall t \in\left(t_{\text {start }}, t_{\text {end }}\right) .
\end{array}
$$

To ensure an accurate and numerically stable evaluation of these quantities it is convenient to express each energy as a function of the dual variables $\sigma$ and $v$ used in the DMH formulation. The application of the BE advancing scheme yields the 


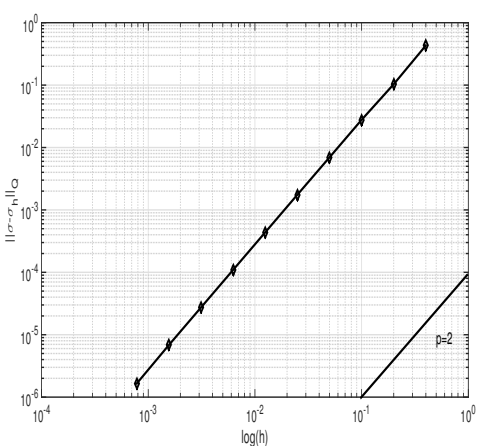

(a) $\left\|\sigma-\sigma_{h}\right\|_{Q}$

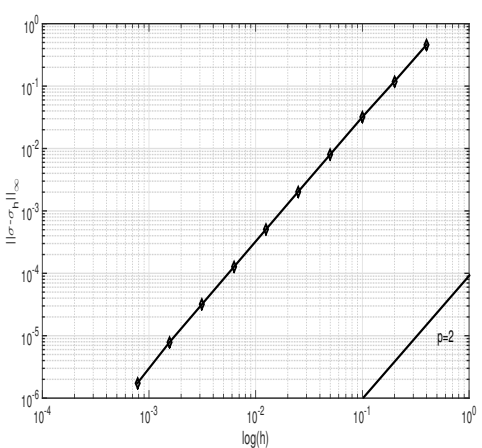

(b) $\left\|\sigma-\sigma_{h}\right\|_{\infty}$

Fig. 11 Validation test $\mathrm{V}_{4}$. Discretization errors for the total stress show that the convergence rate $\left(\mathscr{O}\left(h^{2}\right)\right)$ is optimal in both norms.

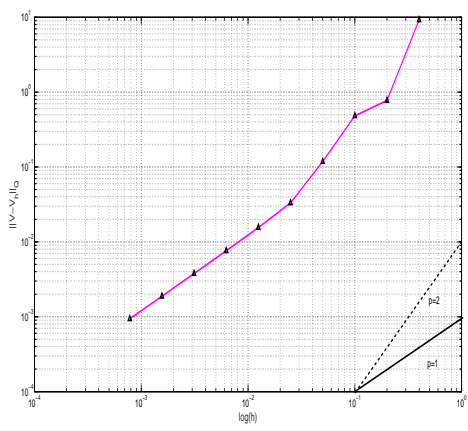

(a) $\left\|v-v_{h}\right\|_{Q}$

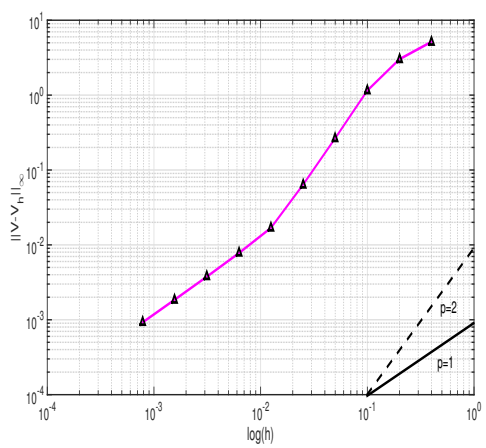

(b) $\left\|v-v_{h}\right\|_{\infty}$

Fig. 12 Validation test $\mathrm{V}_{4}$. Discretization errors for Darcy's velocity show that the convergence rate for higher values of $h$ (and $\Delta t$ ) is close to optimal $\left(\mathscr{O}\left(h^{2}\right)\right.$ ). However, as $h, \Delta t \rightarrow 0$, the asymptotic convergence rate becomes sub-optimal $(\mathscr{O}(h))$ because the time approximation error dominates over the second-order spatial accuracy of the DMH method.

following form of the (approximate) energies at time $t^{i+1}, i=0, \ldots, r-1$ :

$$
\begin{aligned}
& E_{e}^{i+1}=\frac{1}{2}\left(\lambda_{e}+4 \mu_{e}\right)\left\|\varepsilon_{h}^{i+1}(x)\right\|_{0}^{2}, \\
& E_{v}^{i+1}=\frac{1}{2}\left(\lambda_{v}+4 \mu_{v}\right)\left\|\varepsilon_{h}^{i+1}(x)\right\|_{0}^{2}, \\
& E_{p}^{i+1}=\left\|\frac{1}{\sqrt{k\left(\mathscr{P}_{h}^{i+1}(x)\right)}} v_{h}(x)\right\|_{0}^{2},
\end{aligned}
$$

where

$$
\varepsilon_{h}^{i+1}(x)=\frac{1}{A_{e}}\left[\sigma_{h}(x)^{i+1}+B_{e} \mathscr{P}_{h}^{i+1}(x)+p_{h}^{i+1}(x)-\delta \frac{H_{v} \mathscr{P}_{h}^{i}(x)}{\lambda_{e} \Delta t}\right],
$$


having set

$$
A_{e}:=2 \mu_{e}\left(1+\frac{\delta \mu_{v}}{\Delta t \mu_{e}}\right), \quad B_{e}:=1+\frac{\delta \lambda_{v}}{\Delta t \lambda_{e}} .
$$

We notice that the evaluation of (207)-(209) does not require numerical differentiation, in contrast with (204)-(206), and therefore it is expected that the high accuracy provided by the DMH scheme in the approximation of the stress and the pressure variables reflects into the evaluation of the energies.

It is important to recall that the energy estimates in Section 3.6 rely on different time regularity requirements for the volumetric source of linear momentum and for the boundary source of stress, corresponding to $F$ and $g$ in the 1D case, depending on whether $\delta=0$ (poro-elastic model) or $\delta>0$ (poro-visco-elastic model). Thus, we would like to utilize the DMH method to simulate and compare the behavior of the energies characterizing the poro-elastic and poro-visco-elastic models in the presence of data with different regularity in time. To this end, we introduce a discontinuous function of time $\mathscr{G}$ defined as

$$
\mathscr{G}\left(t ; t_{a}, t_{b}\right):=\mathscr{H}\left(t-t_{a}\right)-\mathscr{H}\left(t-t_{b}\right),
$$

where $\mathscr{H}(y-\bar{y})$ is the Heaviside function centered at $\bar{y}$, and $t_{a}$ and $t_{b}$ are such that $t_{\text {start }} \leq t_{a}<t_{b} \leq t_{\text {end }}$, and a smooth function of time $\mathscr{G}_{q}$ defined as

$$
\mathscr{G}_{q}\left(t ; t_{a}, t_{b}\right):=\frac{1}{2}\left[\tanh \left(q\left(t-t_{a}\right)\right)-\tanh \left(q\left(t-t_{b}\right)\right)\right],
$$

which is a double hyperbolic tangent temporal lifting of the function $\mathscr{G}$. Here $q$ is a parameter that controls the slope of the lifting in the neighbourhood of $t=t_{a}$ and $t=t_{b}$. The larger $q$ the steeper the lifting, with $\lim _{q \rightarrow+\infty} \mathscr{G}_{q}\left(t ; t_{a}, t_{b}\right)=\mathscr{G}\left(t ; t_{a}, t_{b}\right)$ for all $t \in\left(t_{\text {start }}, t_{\text {end }}\right)$. Consequently, we can define the functions:

$$
\begin{aligned}
\mathscr{S}\left(x, t ; x_{a}, x_{b}, t_{a}, t_{b}\right) & :=\left[\mathscr{H}\left(x-x_{a}\right)-\mathscr{H}\left(x-x_{b}\right)\right] \mathscr{G}\left(t ; t_{a}, t_{b}\right), \\
\mathscr{S}_{q}\left(x, t ; x_{a}, x_{b}, t_{a}, t_{b}\right) & :=\left[\mathscr{H}\left(x-x_{a}\right)-\mathscr{H}\left(x-x_{b}\right)\right] \mathscr{G}_{q}\left(t ; t_{a}, t_{b}\right),
\end{aligned}
$$

that are both discontinuous in space but that obviously enjoy different regularity in time. A portrait of $\mathscr{S}$ and $\mathscr{S}_{q}$ is provided in Fig. 13 having set $x_{\text {start }}=t_{\text {start }}=0$, $x_{\text {end }}=t_{\text {end }}=1, x_{a}=t_{a}=1 / 3, x_{b}=t_{b}=2 / 3$ and $q=40$.

In the following, the functions $\mathscr{S}$ and $\mathscr{S}_{q}$ (resp. $\mathscr{G}$ and $\mathscr{G}_{q}$ ) are used to investigate how the energies defined in (207)-(209) are influenced by the time regularity in the volumetric (resp. boundary) data for the linear momentum (resp. stress) in one-dimensional poro-elastic and poro-viscoelastic models. All the simulations reported in the following sections are performed by adopting the expression (7) for the permeability $k$, with the same limitation (203) as in Sect. 4.5. Analogously, the characteristic elastic time constant is defined as $\tau_{e}=L \sqrt{\rho / H_{A}}$, with $\rho$ denoting the fluid density (see [5] and [22]), in such a way that the viscous aggregate modulus $H_{V}$, via dimensional analysis, can be estimated as $H_{V}=H_{A} \tau_{e}$. The values of the main biophysical parameters used in the analysis are reported in Tab. 2 whereas the initial and boundary conditions are described for each specific case. The discretization parameters are $\mathscr{K}_{h}=50$ and $N_{T}=200$, so that we have $h=4 \cdot 10^{-2} \mathrm{~cm}$ and $\Delta t=10^{-2} \mathrm{~s}$. The parameters of the square wave with compart support in space and time are $x_{a}=-L / 8=-0.25 \mathrm{~cm}, x_{b}=+L / 8=+0.25 \mathrm{~cm}$, $t_{a}=-T / 8=-0.25 \mathrm{~s}$ and $t_{b}=+T / 8=+0.25 \mathrm{~s}$. 


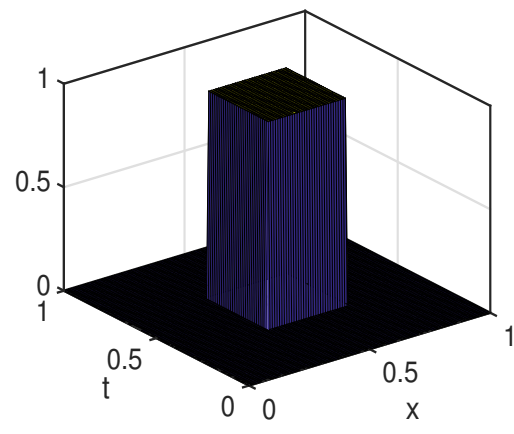

(a) Representation of $\mathscr{S}$

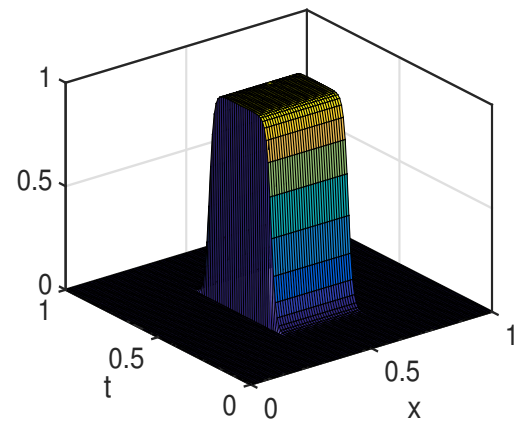

(b) Representation of $\mathscr{S}_{q}$

Fig. 13 Representation of the functions $\mathscr{S}$ (panel (a)) and $\mathscr{S}_{q}$ (panel (b)) in the space-time domain $([1 / 3,2 / 3] \times[1 / 3,2 / 3]) \subset([0, \underline{1]} \times[0, \underline{1]})$ and for $q=40$.

\begin{tabular}{|c|c|c|}
\hline Parameter & Value & Units \\
\hline$x_{\text {start }}$ & -1 & $\mathrm{~cm}$ \\
\hline$x_{\text {end }}$ & +1 & $\mathrm{~cm}$ \\
\hline$L$ & 2 & $\mathrm{~cm}$ \\
\hline$t_{\text {start }}$ & 0 & $\mathrm{~S}$ \\
\hline$t_{\text {end }}$ & 2 & $\mathrm{~S}$ \\
\hline$T$ & 2 & $\mathrm{~S}$ \\
\hline$\mu_{e}$ & 1 & dyne $\mathrm{cm}^{-2}$ \\
\hline$\lambda_{e}$ & 1 & dyne $\mathrm{cm}^{-2}$ \\
\hline$\tau_{e}$ & 1.1547 & $\mathrm{~S}$ \\
\hline$\mu_{v}$ & 1.1547 & dyne $\mathrm{cm}^{-2} \mathrm{~s}$ \\
\hline$\lambda_{v}$ & 1.1547 & dyne $\mathrm{cm}^{-2} \mathrm{~s}$ \\
\hline$H_{A}$ & 3 & dyne $\mathrm{cm}^{-2}$ \\
\hline$H_{V}$ & 3.4641 & dyne $\mathrm{cm}^{-2} \mathrm{~s}$ \\
\hline$C_{c k}$ & 1 & $\mathrm{~cm}^{2}$ \\
\hline$\mu_{f}$ & 1 & $\mathrm{~g} \mathrm{~cm}^{-1} \mathrm{~s}^{-1}$ \\
\hline$k_{r e f}$ & 1 & $\mathrm{~cm}^{3} \mathrm{sg}^{-1}$ \\
\hline$\rho$ & 1 & $\mathrm{~g} \mathrm{~cm}^{-3}$ \\
\hline$\Phi_{0}$ & 0.5 & \\
\hline$\Phi_{\min }$ & 0.125 & \\
\hline$\Phi_{\max }$ & 0.875 & \\
\hline
\end{tabular}

Table 2 Model parameters used for the numerical investigation of the influence of data time regularity on the energies of the solution to poro-elastic and poro-visco-elastic models (see Sections 4.6.1 and 4.6.2).

\subsubsection{Influence of time regularity in the volumetric source of linear momentum}

Let us consider problem (138)-(143) in the spatio-temporal domain

$$
\mathscr{Q}_{T}=\left(x_{\text {start }}, x_{\text {end }}\right) \times\left(t_{\text {start }}, t_{\text {end }}\right)=(-1,1) \times(0,2),
$$


so that $L=2 \mathrm{~cm}$ and $T=2 \mathrm{~s}$. Let us assume that the volumetric sources of linear momentum and mass are given by:

$$
\begin{aligned}
F(x, t) & =\bar{F} \mathscr{S}\left(x, t ; x_{a}, x_{b}, t_{a}, t_{b}\right), \quad \forall(x, t) \in \mathscr{Q}_{T}, \\
S(x, t) & =0, \quad \forall(x, t) \in \mathscr{Q}_{T},
\end{aligned}
$$

respectively, where $\mathscr{S}$ is the square wave function introduced in (212) and $\bar{F}:=$ $0.1 H_{A} / L$ dyne $\mathrm{cm}^{-3}$ is the maximum value of $F$. In addition, let us impose the following initial and boundary conditions:

$$
\begin{aligned}
u\left(x, t_{\text {start }}\right) & =0, & & \forall x \in \Omega, \\
u\left(x_{\text {start }}, t\right) & =u\left(x_{\text {end }}, t\right)=0, & & \forall t \in\left(t_{\text {start }}, t_{\text {end }}\right) \\
p\left(x_{\text {end }}, t\right) & =p\left(x_{\text {end }}, t\right)=0 & & \forall t \in\left(t_{\text {start }}, t_{\text {end }}\right) .
\end{aligned}
$$

We remark that homogeneous boundary conditions are enforced on $\partial \Omega$ for solid displacement and fluid pressure in order to allow the redistribution of stress and fluid across the material. This choice has been made to better single out the sensitivity of the biophysical system to the sole source term $F$. We also remark that $F$ has been chosen in such a way to satisfy the regularity requirements for Lemma 12 but not those for Lemma 13. Figures 14(a) and 14(b) illustrate the simulation results for the poro-elastic model (i.e. without solid viscoelasticity, $\delta=0$ ) and for the poro-visco-elastic model (i.e. with viscoelasticity, $\delta=1$ ). Interestingly:

Case $\delta=0$ : Fig. 14(a) shows two sharp peaks in $E_{p}$ localized around the signal switch-on time $t=0.75 \mathrm{~s}$ and the signal switch-off time $t=1.25 \mathrm{~s}$, demonstrating that the lack of regularity in time of $F$ reflects into a lack of regularity in the fluid energy $E_{p}$ when viscoelasticity is not included in the differential model. Interestingly, the two peaks of $E_{p}$ tend to increase in size as $\Delta t$ tends to zero. On the other hand, the time evolution of the elastic energy $E_{e}$ shows a rapid exponential increase at signal switch-on followed by a similarly fast decay at signal switch-off, because of the lack of memory in system energy storage.

Case $\delta=1$ : Fig. 14(b) shows a remarkably different behavior of $E_{p}$ and $E_{e}$ with respect to what reported in Fig. 14(a). The peak of $E_{p}$ around the signal switch-on time has a much lower intensity than in the case $\delta=0$ (three orders of magnitude smaller) and fluid energy relaxation occurs with two time constants (corresponding to the time interval when the source is on and to the time interval when it is off) that are much larger than in the case $\delta=0$.

The presence of a singularity in $E_{p}$ when $\delta=0$ is further investigated by comparing the results obtained when progressively reducing the time regularity of $F$, as shown in Fig. 15. Specifically, we write $F(x, t)=\bar{F} \mathscr{S}_{q}\left(x, t ; x_{a}, x_{b}, t_{a}, t_{b}\right)$ and we compare the energies obtained when $q=0.1,0.5,1,2.5,5,10,20,40,50,100,+\infty$. Fig. 15 shows how the peaks in $E_{p}$ get higher as $F$ gets sharper (i.e. $q \rightarrow+\infty$ ). In conclusion, when the dynamics is driven by a source $F$ of linear momentum such that $F \in L^{2}\left(t_{\text {start }}, t_{\text {end }} ; L^{2}\left(x_{\text {start }}, x_{\text {end }}\right)\right)$ but not $F_{t} \in L^{2}\left(t_{\text {start }}, t_{\text {end }} ; L^{2}\left(x_{\text {start }}, x_{\text {end }}\right)\right)$, numerical simulations show that:

- if $\delta>0, E_{e}$ and $E_{p}$ are bounded functions of time for all $t \in\left[t_{\text {start }}, t_{\text {end }}\right]$. This confirms experimentally the estimate (131) of Lemma 12; 

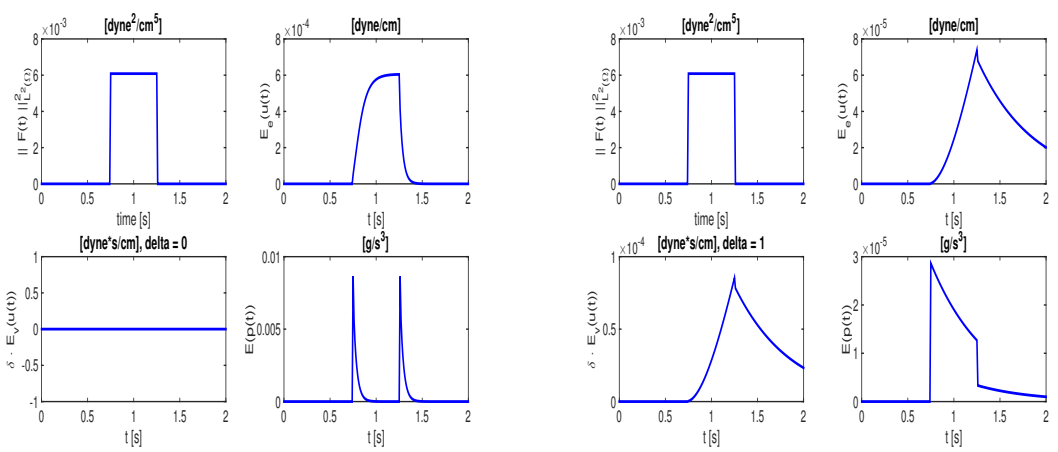

(a) $\delta=0$

(b) $\delta=1$

Fig. 14 Computed energies $\|F(t)\|_{0}^{2}, E_{e}, E_{v}$ and $E_{p}$ in the case where the system is driven by the sole volumetric source $F$ in the linear momentum balance equation. Left panel (a): $\delta=0$; right panel (b): $\delta=1$.
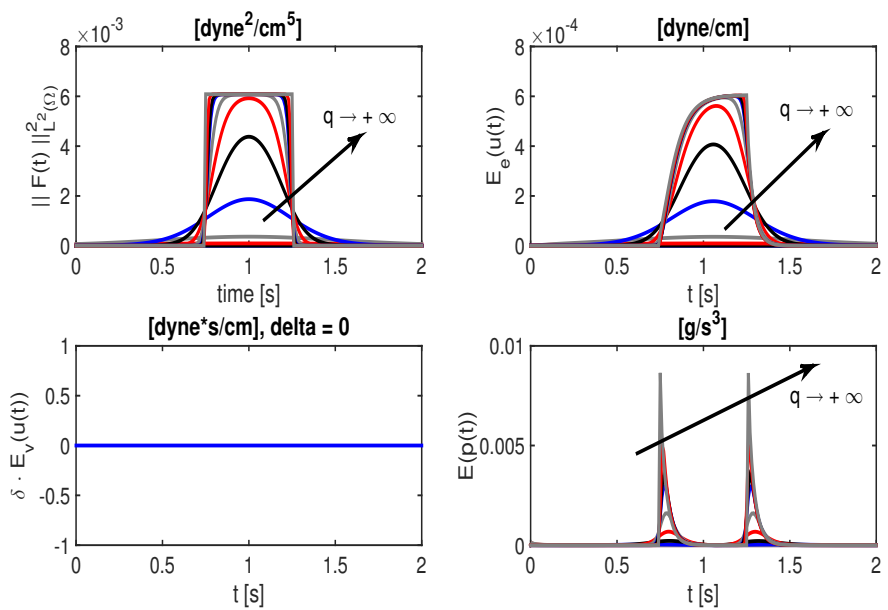

Fig. 15 Computed energies $\|F(t)\|_{0}^{2}, E_{e}, E_{v}$ and $E_{p}$ in the case where the system is driven by the sole volumetric source $F$ in the linear momentum balance equation and $\delta=0$, as a function of the slope parameter $q=0.1,0.5,1,2.5,5,10,20,40,50,100,+\infty$. The value $q=+\infty$ corresponds to the case where $F$ is non-smooth in space and time.

- if $\delta=0, E_{e}$ is a continuous function of time for all $t \in\left[t_{\text {start }}, t_{\text {end }}\right]$ while $E_{p}$ tends to $+\infty$ at $t=0.75 \mathrm{~s}$ and $t=1.25 \mathrm{~s}$ as $\Delta t$ tends to zero. This blow-up of the fluid energy agrees with the fact that $F_{t} \notin L^{2}\left(t_{\text {start }}, t_{\text {end }} ; L^{2}\left(x_{\text {start }}, x_{\text {end }}\right)\right)$ so that the right-hand side of estimate (135) of Lemma 13 cannot be bounded.

The remaining figures of this section illustrate the space-time distributions of various biophysical quantities in the interesting case where $F$ is defined as in (214). The computed displacement $u_{h}^{*}$ is shown in Fig. 16. The left panel refers to the case $\delta=0$ whereas the right panel refers to the case $\delta=1$. In both cases we see that the displacement is a symmetric function with respect to $x=0$ in accordance 
with the applied source and the homogeneous Dirichlet boundary conditions. The behaviour of the displacement in the case without viscoelasticity reflects the exponential increase and decrease of the elastic energy and the same holds in the viscous case where the time constant of increase and decrease of the displacement are remarkably different.

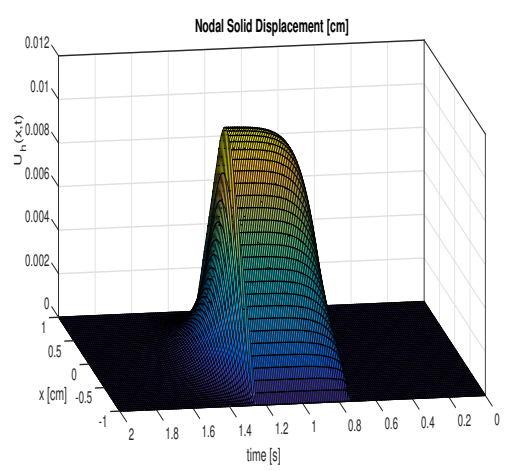

(a) $\delta=0$

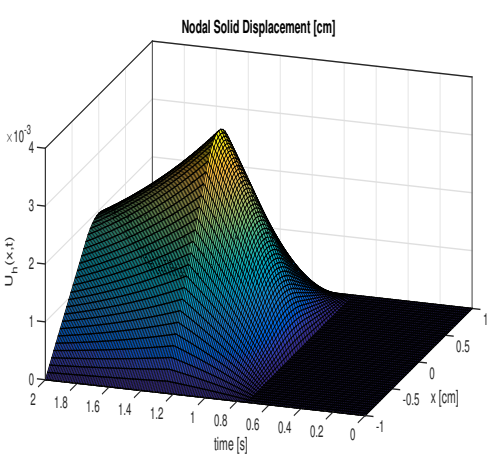

(b) $\delta=1$

Fig. 16 Computed displacement $u_{h}^{*}$ in the case where the system is driven by the sole volumetric source $F$ in the linear momentum balance equation. Left panel (a): $\delta=0$; right panel (b): $\delta=1$.

The computed total stress $\sigma_{h}^{*}$ is the same in both cases $\delta=0$ and $\delta=1$ in accordance with linear momentum balance. Fig. 17 correctly reproduces the piecewise linear spatial variation of the stress within the time interval $[0.75,1.25] \mathrm{s}$, since the slope of $\sigma_{h}$ is negative where $F$ is positive. Fig. 18 shows the distri-

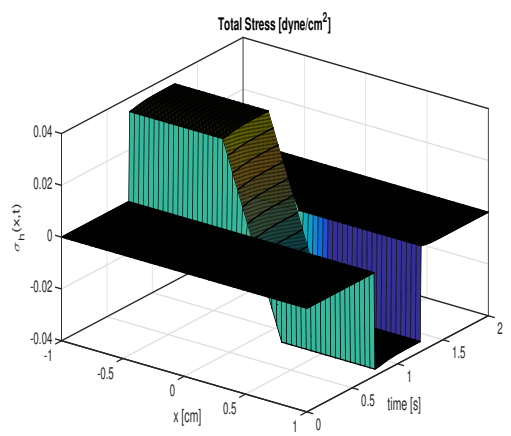

(a) $\delta=0$

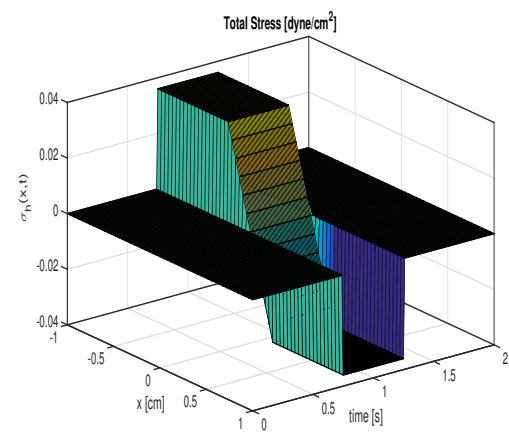

(b) $\delta=1$

Fig. 17 Computed total stress $\sigma_{h}$ in the case where the system is driven by the sole volumetric source $F$ in the linear momentum balance equation. Left panel (a): $\delta=0$; right panel (b): $\delta=1$.

bution of the elastic pressure $\mathscr{P}_{h}$ in the purely elastic and viscoelastic regimes. We remark that $\mathscr{P}_{h}$ is proportional to $-\partial u_{h} / \partial x$; thus, the elastic pressure is an 
odd function with respect to $x=0$ since the displacement is a concave function of position with its maximum at $x=0$. Consistently with what shown in Fig.16(a) and Fig. 16(b) for the displacement, the elastic pressure behaves very differently depending on whether $\delta=0$ or $\delta=1$. The computed fluid pressure $p_{h}^{*}$ (Darcy

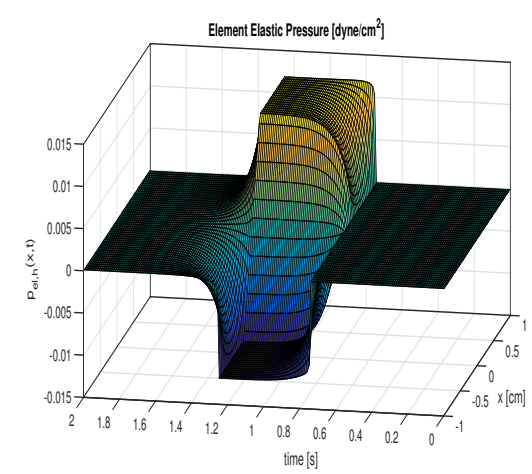

(a) $\delta=0$

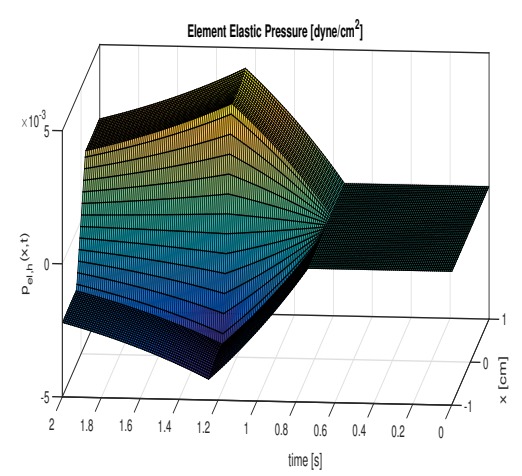

(b) $\delta=1$

Fig. 18 Computed elastic pressure $\mathscr{P}_{h}$ in the case where the system is driven by the sole volumetric source $F$ in the linear momentum balance equation. Left panel (a): $\delta=0$; right panel (b): $\delta=1$.

pressure) is shown in Fig. 19. The behaviour of the variable is the result of the fact that, at each time level, the mass balance equation (139) is supplied with a right-hand side that is proportional to the time derivative of the elastic pressure, since $S=0$ in the present configuration. This explains the various changes of sign of $p_{h}^{*}$ in the region of the space-time domain with $t \geq 0.75 \mathrm{~s}$. In particular, $p_{h}^{*}$ is $>0$ (resp. $<0)$ where $\partial \mathscr{P}_{h} / \partial t$ is $>0$ (resp. $\left.<0\right)$. The computed fluid velocity

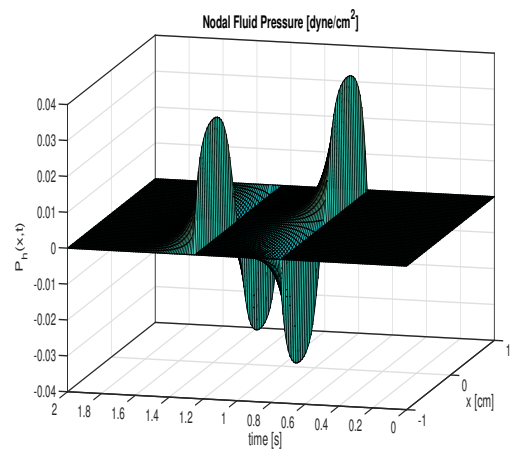

(a) $\delta=0$

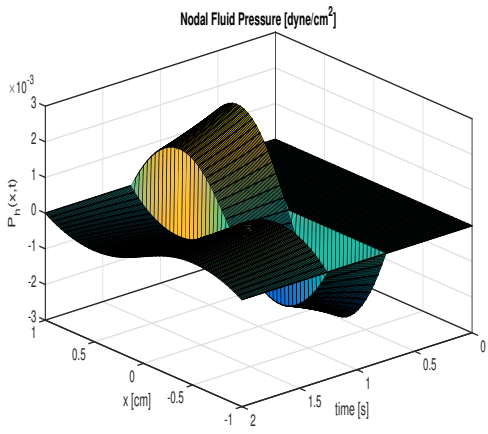

(b) $\delta=1$

Fig. 19 Computed Darcy pressure $p_{h}^{*}$ in the case where the system is driven by the sole volumetric source $F$ in the linear momentum balance equation. Left panel (a): $\delta=0$; right panel (b): $\delta=1$. 
$v_{h}$ (Darcy velocity) is shown in Fig. 20. A remarkable difference between the two regimes (elastic and visco-elastic) can be detected by inspecting the larger values (in module) attained by $v_{h}$ in the case $\delta=0$. This is the same aspect that we noticed in the analysis of the fluid energy $E_{p}$. Also, the smoothness of the velocity is rather different in the two cases, the viscoelastic regime being much more regular than the purely elastic regime. Corner singularities (much larger in the purely elastic case) are due to the homogeneous Dirichlet conditions for the fluid pressure whereas the change of sign of the velocity agrees with Darcy's law and with the spatial distribution of $p_{h}^{*}$. The two final sets of figures refer to the

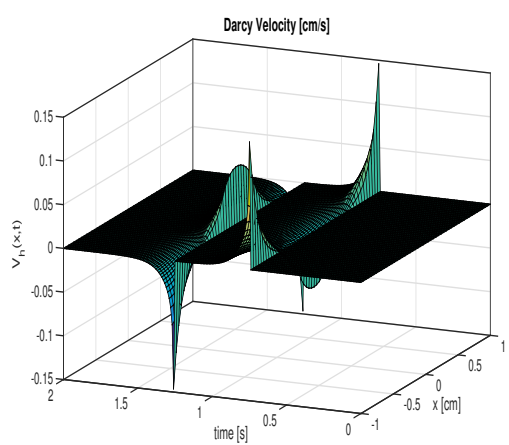

(a) $\delta=0$

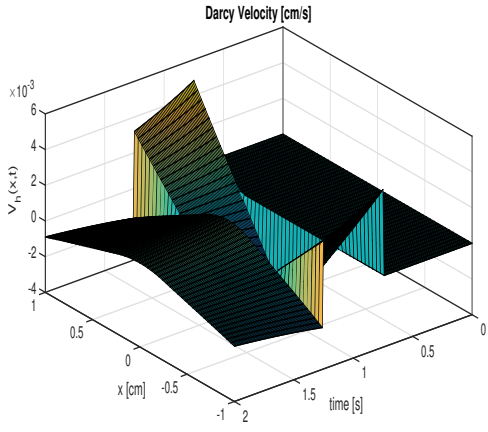

(b) $\delta=1$

Fig. 20 Computed Darcy velocity $v_{h}$ in the case where the system is driven by the sole volumetric source $F$ in the linear momentum balance equation. Left panel (a): $\delta=0$; right panel (b): $\delta=1$.

computed porosity $\phi_{h}$ and permeability $k_{h}$. We recall that these two quantities are a by-product of the DMH simulation and are evaluated using (1) and (7) with the following substitution

$$
\zeta=\frac{\partial u}{\partial x}=-\frac{\mathscr{P}}{\lambda_{e}} .
$$

This avoids the use of numerical differentiation and improves the accuracy of the method. Figs. 21 and 22 look quite similar: this is due to the fact that the applied source is small enough to maintain the nonlinear Carman-Kozeny relation (7) for hydraulic permeability in a neighborhood of $\phi=\phi_{0}$ and $k=k_{r e f}$. In accordance with this observation, we see that the profiles of the two quantities closely follow those of the elastic pressure $\mathscr{P}_{h}$ with a larger deviation from $\phi_{0}\left(k_{r e f}\right)$ in the elastic regime than in the visco-elastic regime. This means that the fluid portion of the mixture varies more considerably in the case of a poro-elastic medium than in the case of a poro-visco-elastic medium.

\subsubsection{Influence of time regularity in the boundary source of stress}

Let us consider problem (138)-(143) in the spatio-temporal domain

$$
\mathscr{Q}_{T}=\left(x_{\text {start }}, x_{\text {end }}\right) \times\left(t_{\text {start }}, t_{\text {end }}\right)=(-1,1) \times(0,2),
$$




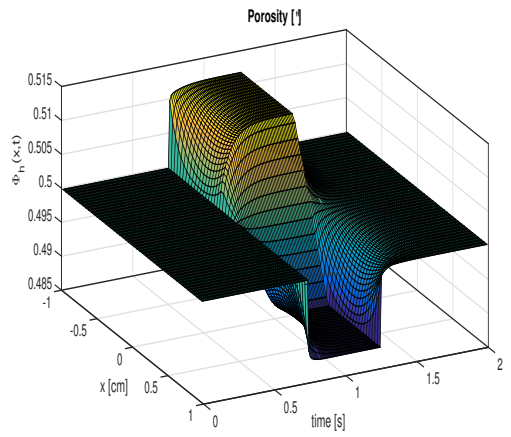

(a) $\delta=0$

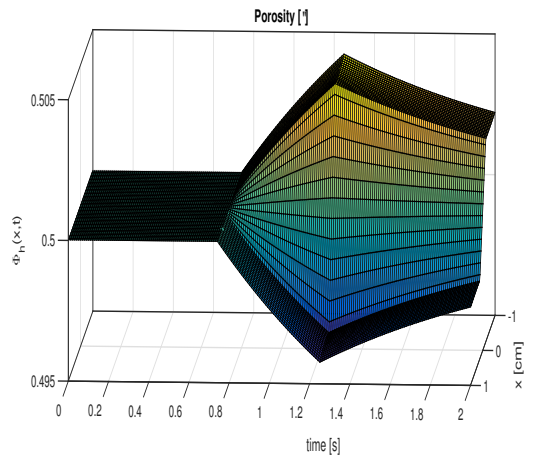

(b) $\delta=1$

Fig. 21 Computed porosity $\phi_{h}$ in the case where the system is driven by the sole volumetric source $F$ in the linear momentum balance equation. Left panel (a): $\delta=0$; right panel (b): $\delta=1$.

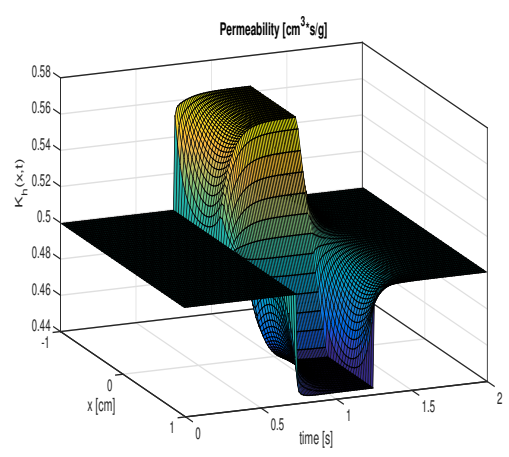

(a) $\delta=0$

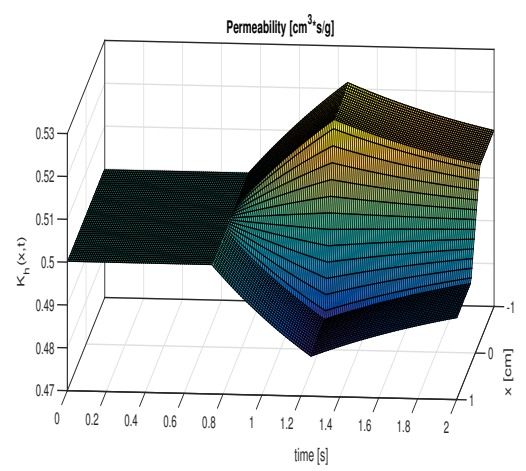

(b) $\delta=1$

Fig. 22 Computed permeability $k_{h}$ in the case where the system is driven by the sole volumetric source $F$ in the linear momentum balance equation. Left panel (a): $\delta=0$; right panel (b): $\delta=1$.

so that $L=2 \mathrm{~cm}$ and $T=2 \mathrm{~s}$. Let us study the problem in the absence of volumetric sources of linear momentum and mass, namely:

$$
\begin{aligned}
F(x, t)=0 & \forall(x, t) \in \mathscr{Q}_{T}, \\
S(x, t)=0, & \forall(x, t) \in \mathscr{Q}_{T},
\end{aligned}
$$

and let us impose the following initial and boundary conditions:

$$
\begin{aligned}
u\left(x, t_{\text {start }}\right) & =0, & & \forall x \in \Omega, \\
u\left(x_{\text {start }}, t\right) & =p\left(x_{\text {start }}, t\right)=v n\left(x_{\text {end }}, t\right)=0, & & \forall t \in\left(t_{\text {start }}, t_{\text {end }}\right) \\
\sigma n\left(x_{\text {end }}, t\right) & =\bar{g} \mathscr{G}\left(t ; t_{a}, t_{b}\right) & & \forall t \in\left(t_{\text {start }}, t_{\text {end }}\right),
\end{aligned}
$$

where $\mathscr{G}$ is the square wave introduced in $(210)$ and $\bar{g}:=0.01 H_{A} \mathrm{dyne} \mathrm{cm}^{-2}$ is the maximum value of $g$. We remark that now the problem dynamics is solely driven by the boundary source term for the stress and that $g$ has been chosen in such a 
way to satisfy the requirements of Lemma 12 but not those for Lemma 13. Figures 23-30 illustrate the simulation results for the poro-elastic model (i.e. without solid viscoelasticity, $\delta=0$ ) and for the poro-visco-elastic model (i.e. with viscoelasticity, $\delta=1)$. Interestingly:

Case $\delta=0$ : Fig. 23(a) shows two sharp peaks in $E_{p}$ localized around the signal switch-on time $t=0.75 \mathrm{~s}$ and the signal switch-off time $t=1.25 \mathrm{~s}$, demonstrating that also a lack of regularity in time of $g$ reflects into a lack of regularity in the fluid energy $E_{p}$ when viscoelasticity is not included in the differential model, as observed in the previous subsection for $F$. Also in this case, the peaks in $E_{p}$ tend to increase in size as $\Delta t$ tends to zero. However, unlike the case in which $F$ lacks regularity, when the system is driven by the non-regular boundary term $g$, the two peaks of $E_{p}$ do not have equal size, suggesting a different response of the system at switch-on and switch-off times. We also note that the time evolution of the elastic energy $E_{e}$ shows a very similar behavior to that observed when forcing the system with $F$.

Case $\delta=1$ : When comparing Fig. 23(b) and Fig. 23(a), we notice that the peak of $E_{p}$ around the signal switch-on time has a lower intensity than in the case $\delta=0$ (one order of magnitude smaller) and fluid energy relaxation occurs with two time constants (corresponding to the time interval when the source is on and to the time interval when it is off) that are much larger than in the case $\delta=0$.
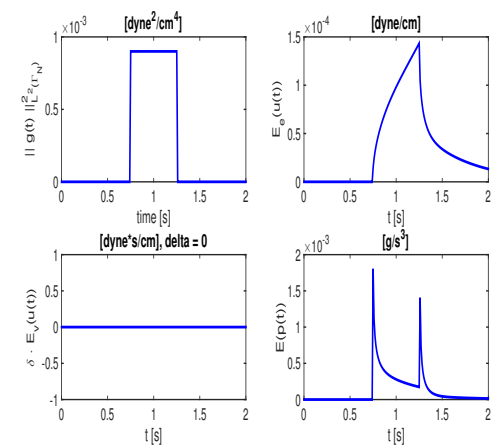

(a) $\delta=0$
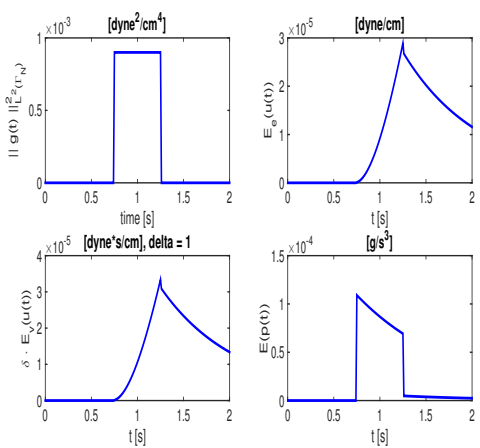

(b) $\delta=1$

Fig. 23 Computed energies $\|g(t)\|_{0}^{2}, E_{e}, E_{v}$ and $E_{p}$ in the case where the system is driven by the sole boundary source $g$ in the linear momentum balance equation. Left panel $(\mathrm{a}): \delta=0$; right panel (b): $\delta=1$.

The presence of a singularity in $E_{p}$ when $\delta=0$ is further investigated by comparing the results obtained when progressively reducing the time regularity of $g$, as shown in Fig. 24. Specifically, we write $g(t)=\bar{g} \mathscr{G}_{q}\left(t ; t_{a}, t_{b}\right)$ and we compare the energies obtained when $q=0.1,0.5,1,2.5,5,10,20,40,50,100,+\infty$. Fig. 24 shows how the peaks in $E_{p}$ get higher as $g$ gets sharper (i.e. $q \rightarrow+\infty$ ). In conclusion, when the dynamics is driven by a source $g$ of linear momentum such that $g \in L^{2}\left(t_{\text {start }}, t_{\text {end }}\right)$ but not $g_{t} \in L^{2}\left(t_{\text {start }}, t_{\text {end }}\right)$, numerical simulations show that: 

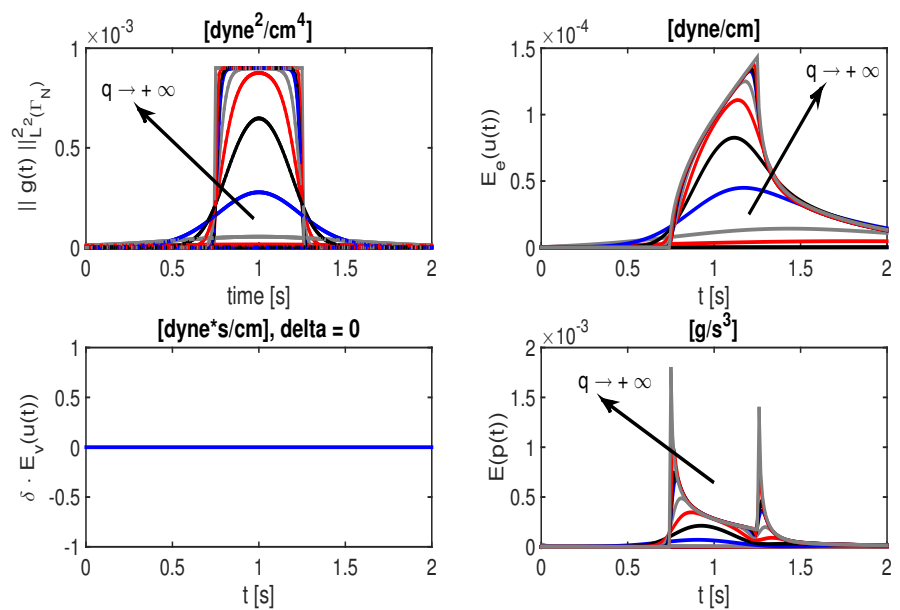

Fig. 24 Computed energies $\|g(t)\|_{0}^{2}, E_{e}, E_{v}$ and $E_{p}$ in the case where the system is driven by the sole boundary source $g$ in the linear momentum balance equation and $\delta=0$, as a function of the slope parameter $q=0.1,0.5,1,2.5,5,10,20,40,50,100,+\infty$. The value $q=+\infty$ corresponds to the case where $g$ is non-smooth in time.

- if $\delta>0, E_{e}$ and $E_{p}$ are bounded functions of time for all $t \in\left[t_{\text {start }}, t_{\text {end }}\right]$. This confirms experimentally the estimate (131) of Lemma 12;

- if $\delta=0, E_{e}$ is a continuous function of time for all $t \in\left[t_{\text {start }}, t_{\text {end }}\right]$ while $E_{p}$ tends to $+\infty$ at $t=0.75 \mathrm{~s}$ and $t=1.25 \mathrm{~s}$ as $\Delta t$ tends to zero. This blow-up of the fluid energy agrees with the fact that $g_{t} \notin L^{2}\left(t_{\text {start }}, t_{\text {end }}\right)$ so that the right-hand side of estimate (135) of Lemma 13 cannot be bounded.

The remaining figures of this section illustrate the space-time distributions of various biophysical quantities in the interesting case where $g$ is defined as in (223). In order to understand whether the peaks in $E_{p}$ actually correspond to blow-ups, it is particularly interesting to compare the space-time plots of the fluid pressure $p_{h}$ and the Darcy velocity $v_{h}$ (approximation of $-k \partial p / \partial x$ ). Their behavior is remarkably different depending on whether $\delta=0$ or $\delta=1$, exhibiting a similar trend to that observed for the energy $E_{p}$. We also notice that, in the case $\delta=0$, the elastic pressure $\wp_{h}$ (approximation of $-H_{A} \partial u / \partial x$ ) is discontinuous in time and this implies that the term $\partial u_{t} / \partial x$ is not defined in the strong sense and that a weaker definition of the solution is needed in the case $\delta=0$.

\section{Conclusions}

The study presented in this article synergistically combines theoretical and numerical analyses to investigate the main features of the solutions to initial-boundary value problems for nonlinear systems of partial differential equations often utilized to describe the motion of a fluid through an elastic or viscoelastic porous material. Our study identifies the presence of viscoelasticity in the solid phase as a major determinant in the behavior of the solutions. From the theoretical viewpoint, 


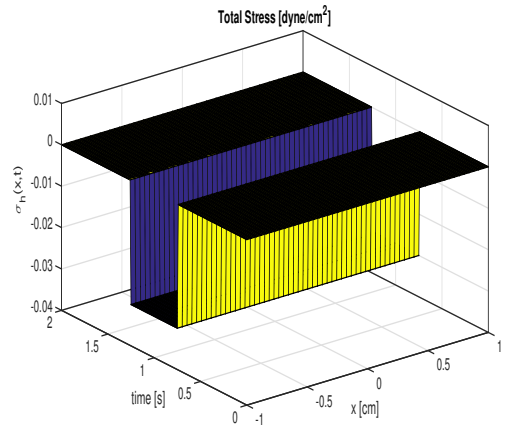

(a) $\delta=0$

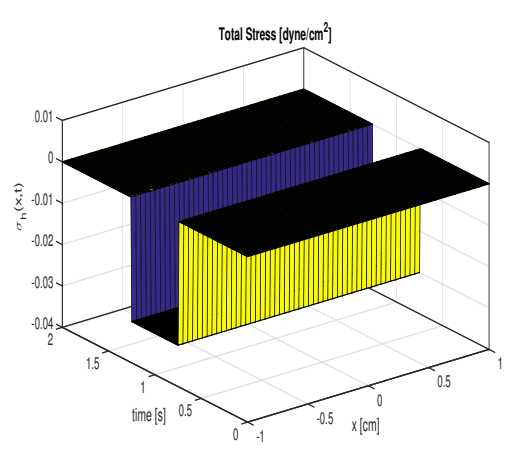

(b) $\delta=1$

Fig. 25 Computed total stress $\sigma_{h}$ in the case where the system is driven by the sole boundary source $g$ in the linear momentum balance equation. Left panel (a): $\delta=0$; right panel (b): $\delta=1$.

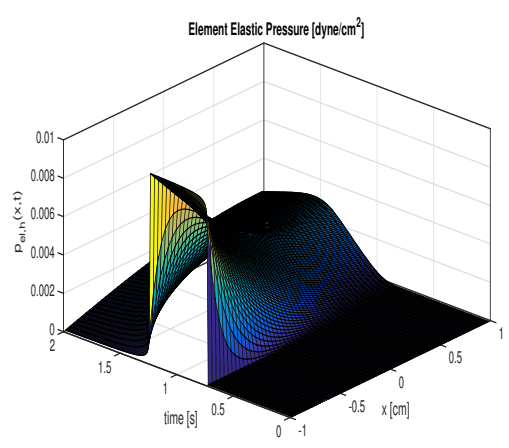

(a) $\delta=0$

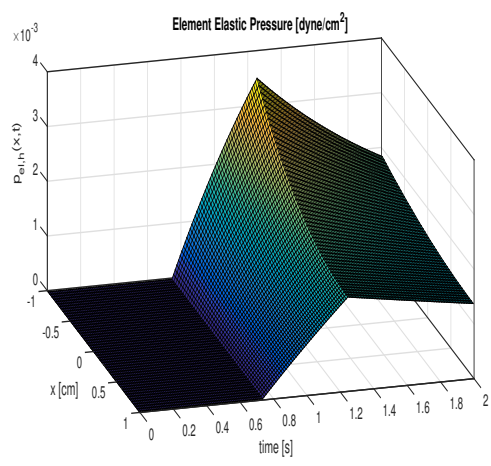

(b) $\delta=1$

Fig. 26 Computed elastic pressure $\mathscr{P}_{h}$ in the case where the system is driven by the sole boundary source $g$ in the linear momentum balance equation. Left panel (a): $\delta=0$; right panel (b): $\delta=1$.

existence of solutions to poro-visco-elastic models can be proved under less restrictive assumptions for data regularity when compared to the purely elastic case. From the numerical viewpoint, the convergence of the computational scheme is faster to be attained for poro-visco-elastic models when compared to their purely elastic counterpart, as a consequence of the fact that solutions are smoother when viscoelasticity is present.

The energy estimates predicted by the theory are confirmed by the numerical experiments when the data are sufficiently regular. Interestingly, in the purely elastic case, when the data do not enjoy sufficient time regularity for the estimates to hold, the numerical experiments actually provide clues of energy blow-up, since: (i) peaks appear in the energy $E_{p}$ in correspondence to the time discontinuity of the data; (ii) the peaks get higher as the time discretization parameter tends to zero; and (iii) the behaviors of fluid pressure and Darcy velocity are much less smooth in the purely elastic case than in the viscoelastic case. 


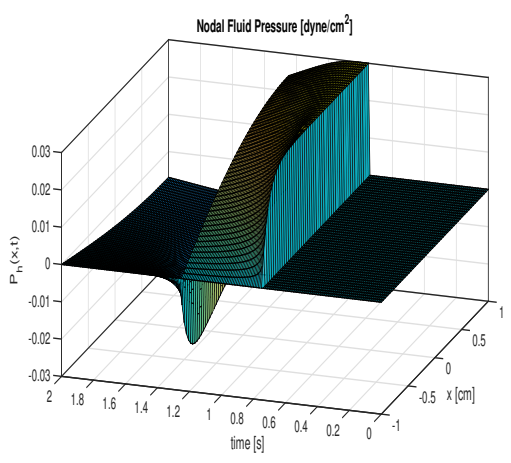

(a) $\delta=0$

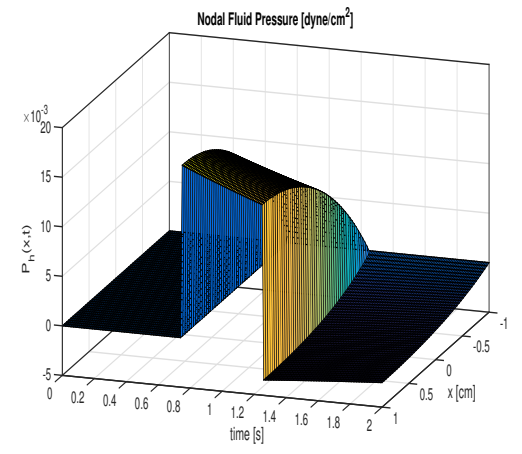

(b) $\delta=1$

Fig. 27 Computed Darcy pressure $p_{h}$ in the case where the system is driven by the sole boundary source $g$ in the linear momentum balance equation. Left panel (a): $\delta=0$; right panel (b): $\delta=1$.

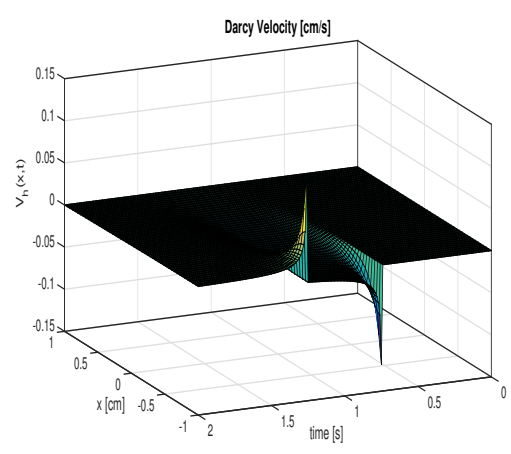

(a) $\delta=0$

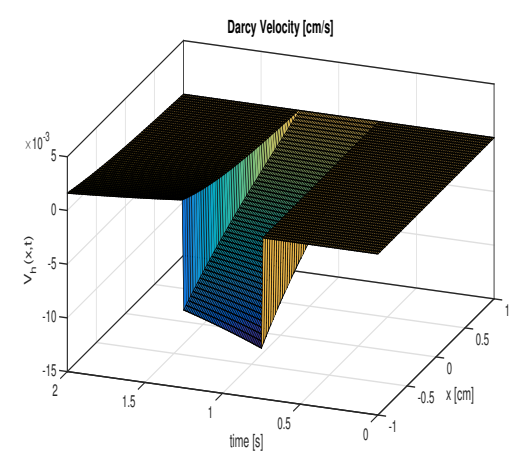

(b) $\delta=1$

Fig. 28 Computed Darcy velocity $v_{h}$ in the case where the system is driven by the sole boundary source $g$ in the linear momentum balance equation. Left panel (a): $\delta=0$; right panel (b): $\delta=1$.

These findings are extremely interesting from the application viewpoint. As mentioned in the introduction, we focused on the role played by viscoelasticity on the regularity of the solutions because of its importance in the modeling of biological tissues, as the viscoelastic tone varies with age or disease status. Our findings suggest that the lack of viscoelasticity may increase the susceptibility of the tissue to localized damage (due to irregularity in the Darcy velocity and peaks in the fluid energy) as volumetric sources of linear momentum and/or boundary sources of traction experience sudden changes in time. We are currently working on applying these concepts to investigate the causes of hemorrhages in the optic nerve head $(\mathrm{ONH})$ tissue, where the intraocular pressure (IOP) acts as a boundary source of traction [9]. Sudden changes in IOP physiologically occur with changes between day and night. Our theoretical findings lead us to hypothesize that even these physiological changes in IOP might induce pathological changes in the hemodynamics of the $\mathrm{ONH}$ tissue if the viscoelasticity provided by the collagen fibers is not in- 


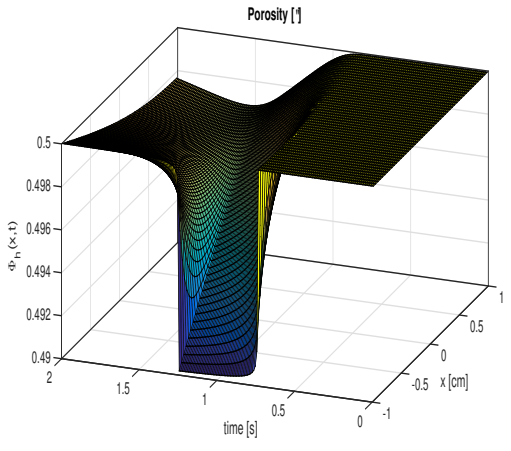

(a) $\delta=0$

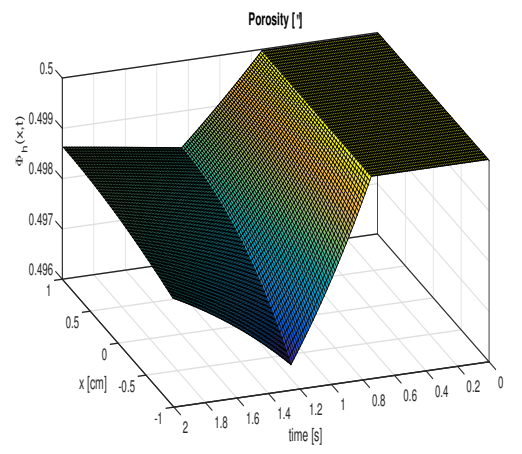

(b) $\delta=1$

Fig. 29 Computed porosity $\phi_{h}$ in the case where the system is driven by the sole boundary source $g$ in the linear momentum balance equation. Left panel (a): $\delta=0$; right panel (b): $\delta=1$.

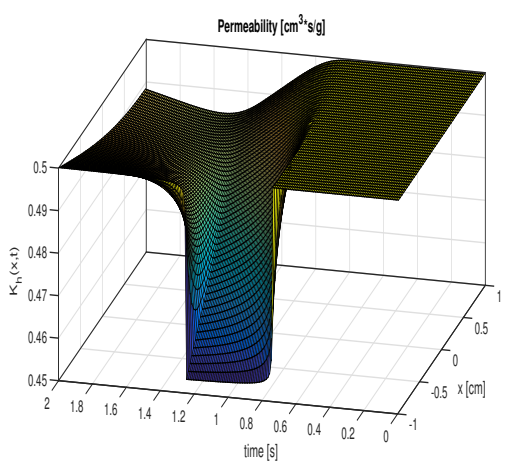

(a) $\delta=0$

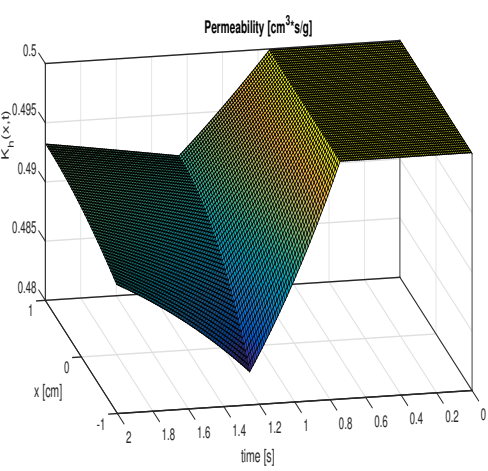

(b) $\delta=1$

Fig. 30 Computed permeability $k_{h}$ in the case where the system is driven by the sole boundary source $g$ in the linear momentum balance equation. Left panel (a): $\delta=0$; right panel (b): $\delta=1$.

tact. Similar considerations may be applicable to other biological tissues as well as to bio-engineered tissues for application in Regenerative Medicine [33].

Interestingly, our findings might also be useful to understand the consequences of gravitational changes on human tissues. As a matter of fact, sudden changes in gravitational acceleration, such as those experienced by astronauts during missions, translate into sudden changes in the volumetric source of linear momentum, which might increase tissue vulnerability to damage, as shown by our analysis. These considerations are particularly relevant for the $\mathrm{ONH}$ tissue, whose pathological changes have been associated with the visual impairments/intracranial pressure (VIIP) syndrome affecting many crew members during and after long-duration space flights [36].

Based on the above considerations, we believe that the present article constitutes a first attempt to combine in a novel cross-disciplinary unified framework the theoretical analysis of nonlinear models in Continuum Mechanics, the development of multi-field Finite Element discretization schemes and the computer sim- 
ulation of the Mechanobiological properties of Tissues and Materials. Next steps of this research will be devoted to considering the problem of uniqueness of solutions, to extending the numerical approach to multi-dimensional geometries and to validating the proposed model against available data in human tissues such as those investigated in [9].

\section{Compliance with Ethical Standards}

Funding: The first author was partially supported by the National Science Foundation with grant NSF-DMS 1312801. The second author was partially supported by the National Science Foundation with grants NSF-DMS 1134731 and 1224195 and by the chair Gutenberg funds of the Cercle Gutenberg of the Region Alsace (France). The fourth author was partially supported by the National Science Foundation with grant NSF-DMS-1504697.

Conflict of Interest: The third author has received a research grant from Micron Technologies Italia, Vimercate (MB, Italy).

Acknowledgements The authors would like to thank A. L. Mazzucato for her helpful discussions of elliptic regularity, and insight into the literature.

\section{References}

1. R. P. Araujo and D. L. Sean McElwain. A mixture theory for the genesis of residual stresses in growing tissues I: a general formulation. SIAM J. Appl. Math., 65(4):1261-1284, 2005.

2. D.N. Arnold, and F. Brezzi. Mixed and nonconforming finite element methods: implementation, postprocessing and error estimates, Math. Modeling and Numer. Anal., 19(1) (1985), pp. 7-32.

3. M.A. Biot, General theory of three-dimensional consolidation, J. Appl. Phys., 12(2) (1941), pp. 155-164.

4. F. Brezzi and M. Fortin. Mixed and Hybrid Finite Element Methods. Springer Verlag, New York, 1991.

5. S. Canic, J. Tambaca, G. Guidoboni, A. Mikelic, C. J. Hartley, and D. Rosenstrauch Modeling Viscoelastic Behavior of Arterial Walls and Their Interaction with Pulsatile Blood Flow, SIAM J. Appl. Math., 67(1) (2006), pp. 164-193.

6. Y. Cao, S. Chen, and A.J. Meir, Analysis and numerical approximations of equations of nonlinear poroelasticity, DCDS-B, 18 (2013), pp. 1253-1273.

7. Y. Cao, S. Chen, and A.J. Meir, Steady flow in a deformable porous medium, Math. Meth. Appl. Sci., 37 (2014), pp. 1029-1041.

8. Y. Cao, S. Chen, and A.J. Meir, Quasilinear Poroelasticity: Analysis and Hybrid Finite Element Approximation, Num. Meth. PDE, published online (2014) DOI: 10.1002/num.21940.

9. P. Causin, G. Guidoboni, A. Harris, D. Prada, R. Sacco, and S. Terragni, A poroelastic model for the perfusion of the lamina cribrosa in the optic nerve head, Math. Biosci., 257 (2014), pp. 33-41.

10. P. Causin, and R. Sacco, A Discontinuous Petrov-Galerkin Method with Lagrangian Multipliers for Second Order Elliptic Problems, SIAM J. Numer. Anal., 43(1) (2005), pp. 280-302.

11. D. Chapelle, J. Sainte-Marie, J.-F. Gerbeau, I. Vignon-Clementel, A poroelastic model valid in large strains with applications to perfusion in cardiac modeling, Comput. Mech., 46(1) (2010), pp. 91-101.

12. P.G. Ciarlet, Three-dimensional elasticity, Vol. 1, Elsevier, 1988.

13. B. Cockburn, B. Dong, J. Guzmán, M. Restelli and R. Sacco, A Hybridizable Discontinuous Galerkin Method for Steady-State Convection-Diffusion-Reaction Problems, SIAM J. Sci. Comput., 31(5) (2009), pp. 3827-3846. 
14. B. Cockburn and J. Gopalakrishnan, A Characterization of Hybridized Mixed Methods for Second Order Elliptic Problems, SIAM J. Numer. Anal., 42(1) (2004), pp. 283-301.

15. B. Cockburn, J. Gopalakrishnan, and R. Lazarov, Unified hybridization of discontinuous Galerkin, mixed, and continuous Galerkin methods for second order elliptic problems, SIAM J. Numer. Anal., 47(2) (2009), pp. 1319-1365.

16. S.C. Cowin, Bone poroelasticity, J. Biomech., 32(3) (1999), pp. 217-238.

17. E. Detournay and A.H.-D. Cheng, Fundamentals of poroelasticity, Chapter 5 in Comprehensive Rock Engineering: Principles, Practice and Projects, Vol. II, Analysis and Design Method, ed. C. Fairhurst, Pergamon Press, (1993), pp. 113-171.

18. L. Evans, Partial Differential Equations, 2nd Ed., AMS, Graduate Studies in Mathematics, $19,2010$.

19. M. Farhloul. A mixed finite element method for the Stokes equations. Numer. Methods Partial Differ. Equ., 10(5), 591-608, 1994.

20. M. Farhloul and M. Fortin. A New Mixed Finite Element for the Stokes and Elasticity Problems. SIAM J. Numer. Anal., 30(4), 971-990, 1993.

21. A. J. H. Frijns. A four-component mixture theory applied to cartilaginous tissues: Numerical modelling and experiments. Thesis (Dr.ir.)-Technische Universiteit Eindhoven (The Netherlands) 2000.

22. Y.C. Fung, Biomechanics: mechanical properties of living tissues, Springer-Verlag, New York, 1993.

23. F. J. Gaspar and F. J. Lisbona and P. N. Vabishchevich. Finite difference schemes for poro-elastic problems. Comput. Methods Appl. Math., 2, 132-142, 2002.

24. F. J. Gaspar and F. J. Lisbona and P. N. Vabishchevich. A finite difference analysis of Biot's consolidation model. Appl. Numer. Math., 44, 487-506, 2003.

25. L. R. Herrmann. Elasticity equations for incompressible and nearly incompressible materials by a variational theorem. AIAA Journal, 3(10):1896-1900, 1965.

26. C.T. Hsu and P. Cheng, Thermal dispersion in a porous medium, Int. J. Heat Mass Tran., $33(8)(1990)$, pp. $1587-1597$.

27. T. J. R. Hughes. The Finite Element Method: Linear Static and Dynamic Finite Element Analysis. Prentice-Hall, 1987.

28. J.M. Huyghe, T. Arts, D.H. van Campen and R.S. Reneman, Porous medium finite element model of the beating left ventricle, Am. J. Physiol., 262 (1992), pp. 1256-1267.

29. S. Kesavan, Topics in Functional Analysis and Applications, New Age International Publishers, 1989.

30. S. M. Klisch. Internally constrained mixtures of elastic continua. Math. Mech. Solids, 4:481-498, 1999.

31. J. Korsawe and G. Starke and W. Wang and O. Kolditz. Finite element analysis of poroelastic consolidation in porous media: Standard and mixed approaches. Computer Methods in Applied Mechanics and Engineering, 195(9-12):1096 - 1115, 2006.

32. W.M. Lai, J.S. Hou and V.C. Mow, A triphasic theory for the swelling and deformation behaviors of articular cartilage, ASME J. Biomech. Eng., 113 (1991), pp. 245-258.

33. R. Langer, Perspectives and Challenges in Tissue Engineering and Regenerative Medicine, Advanced Materials, 21(32-33), pp. 3235-3236 (2009).

34. G. Lemon, J. R. King, H. M. Byrne, O. E. Jensen, and K. M. Shakesheff. Mathematical modelling of engineered tissue growth using a multiphase porous flow mixture theory. $J$. Math. Biol., 52:571-594, 2006.

35. R. W. Lewis and B. A. Schrefler. The Finite Element Method in the Static and Dynamic Deformation and Consolidation of Porous Media. Wiley, 1998.

36. T.H. Mader, C.R. Gibson, A.F. Pass, L.A. Kramer, A.G. Lee, J. Fogarty, W.J. Tarver, J.P. Dervay, D.R. Hamilton, A.E. Sargsyan, J.L. Phillips, D. Tran, W. Lipsky, J. Choi, C. Stern, R. Kuyumjian, J.D. Polk. Optic Disc Edema, Globe Flattening, Choroidal Folds, and Hyperopic Shifts Observed in Astronauts after Long-duration Space Flight, Opthalmology, 118 (10) (2011), pp. 2058-2069.

37. A. L. Mazzucato and V. Nistor, Well-posedness and Regularity for the Elasticity Equations with Mixed Boundary Conditions on Polyhedral Domains and Domains with Cracks, Arch. Ration. Mech. An., 195 (2010), pp. 25-73.

38. V.C. Mow, S.C. Kuei, W.M. Lai and C.G. Armstrong, Biphasic creep and stress relaxation of articular cartilage in compression: theory and experiments, ASME J. Biomech. Eng., 102 (1980), pp. 73-84. 
39. S. Nicaise, About the Lamé System in a Polygonal or a Polyhedral Domain and a Coupled Problem between the Lamé System and the Plate Equation I: Regularity of Solutions, Annali della Scuola Normale Superiore di Pisa, Classe di Scienze 4 e série, 19 (1992), pp. 327-361.

40. S. Owczarek, A Galerkin method for Biot consolidation model, Math. Mech. Solids, 15 (2010), pp. 42-56

41. P. J. Phillips and M. F. Wheeler. A coupling of mixed and discontinuous Galerkin finiteelement methods for poroelasticity. Computational Geosciences, 12(4):417-435, 2008.

42. P. J. Phillips and M. F. Wheeler. Overcoming the problem of locking in linear elasticity and poroelasticity: an heuristic approach. Computational Geosciences, 13(1):5-12, 2009.

43. L. Preziosi and A. Tosin. Multiphase modelling of tumour growth and extracellular matrix interaction: mathematical tools and applications. J. Math. Biol., 58:625-656, 2009.

44. A. Quarteroni, R. Sacco, and F. Saleri Numerical Mathematics, Springer-Verlag Berlin Heidelberg, Texts in Applied Mathematics, 37 (2007).

45. A. Quarteroni and A. Valli. Numerical Approximation of Partial Differential Equations. Springer-Verlag New York, Berlin (1994).

46. P.A. Raviart and J.M. Thomas. A mixed finite element method for second order elliptic problems. In I. Galligani and E. Magenes, editors, Mathematical Aspects of Finite Element Methods,I. Springer-Verlag, Berlin, 1977.

47. S. Rempel and B.-W. Schulze, Mixed Boundary Value Problems for Lamé's System in Three Dimensions, Math. Nachr. 119 (1984), pp. 265-290.

48. J.E. Roberts and J.M. Thomas. Mixed and hybrid methods. In P.G. Ciarlet and J.L. Lions, editors, Finite Element Methods, Part I. North-Holland, Amsterdam, 1991. Vol.2.

49. G. Savaré Regularity and perturbation results for mixed second order elliptic problems Comm. PDE, 22 (1997)

50. R.E. Showalter, Monotone Operators in Banach Space and Nonlinear Partial Differential Equations, AMS, Mathematical Surveys and Monographs, 49, 1996.

51. R.E. Showalter, Diffusion in poro-elastic media, J. Math. Anal. Appl., 251 (2000), pp. 310-340.

52. R.E. Showalter, Diffusion in poro-platic media, Math. Methods Appl. Sci. , 27 (2004), pp. 2131-2151.

53. D. E. Stewart, Dynamics with Inequalities: Impacts and Hard Constraints, SIAM, 2011.

54. N. Su and R.E. Showalter, Partially saturated flow in a poroelastic medium, DCDS-B, 1 (2001), pp. 403-420.

55. A. Zenisek, The existence and uniqueness theorem in Biot's consolidation theory, Appl. Math., 29 (1984), pp. 194-211.

56. O. C. Zienkiewicz and R. L. Taylor The finite element method. Fifth edition, WILEY-VCH Verlag (2002). 